\title{
TIME MINIMAL CONTROL OF BATCH REACTORS
}

\author{
B. BONNARD AND G. LAUNAY
}

\begin{abstract}
In this article we consider a control system modelling a batch reactor in which three species $X_{1}, X_{2}, X_{3}$ are reacting according to the scheme $X_{1} \rightarrow X_{2} \rightarrow X_{3}$, each reaction being irreversible. The control is the temperature $T$ of the reactions or the derivative of this temperature with respect to time. The terminal constraint is to obtain a given concentration of the product $X_{2}$ at the end of the batch. The objective of our study is to introduce and to apply all the mathematical tools to compute the time optimal control as a closed-loop function. This work can be used to optimize the yield of chemical batch reactors.
\end{abstract}

\section{INTRODUCTION}

Until now, the chemical batch reactors are mainly operating at constant temperature. Substantial gain in the yield of such reactors can obviously be obtained by controlling the temperature during the batch. This leads to optimal control problems. Recent developments in geometric optimal control allow to handle the mathematical complexity of such problems.

In [3], the authors consider the time minimal problem for a batch reactor in which three species $X_{1}, X_{2}, X_{3}$ are reacting according to the scheme $X_{1} \rightarrow$ $X_{2} \rightarrow X_{3}$, each reaction being irreversible and of the first order, while the final constraint is to obtain a given ratio of the two concentrations of $X_{1}, X_{2}$ and the control is the derivative of the temperature with respect to time. Due to symmetry the problem can be reduced to a time minimal control problem for a planar system and the time minimal control is computed as a closed-loop function. The objective of this article is to generalize this analysis for a network of the form $X_{1} \rightarrow X_{2} \rightarrow X_{3}$, each reaction being irreversible but of any order, while the terminal constraint is now to obtain a desired production of the intermediate specie $X_{2}$. If the control is the derivative of the temperature with respect to time, this leads to a time minimal control problem for a system in $\mathbb{R}^{3}$ much more complex than a planar one.

The main objective of our study is to present general techniques and results to handle the problem; they are mainly threefold:

Bernard Bonnard: Université de Bourgogne, Laboratoire de Topologie, UMR 5584, BP 400, 21011 Dijon Cedex, France. Email: bbonnard@satie.u-bourgogne.fr.

Geneviève Launay: INRIA Rocquencourt, Domaine de Voluceau, BP 105, 78153 Le Chesnay Cedex, France. Email: launay@pascal.inria.fr.

Received by the journal November 18, 1997. Accepted for publication August 24, 1998.

(C) Société de Mathématiques Appliquées et Industrielles. Typeset by $\mathrm{IAT}_{\mathrm{EX}} \mathrm{X}$. 
- first we compute the time optimal control as a closed-loop function near the terminal constraint. This analysis is intricated; it has motivated and it uses the mathematical developments of [5], [14],

- secondly we need global estimates concerning the number of switchings of an optimal control,

- endly we have to introduce the concept of focal points and give an algorithm to compute such points. This part is related to techniques introduced in [4].

The combination of the three previous analysis with numerical simulations allows to compute the optimal synthesis in many situations. Also it can be applied to more general reaction scheme to get closed-loop sub-optimal control laws.

This work was motivated by practical control problem and is currently implemented on a batch reactor located at Caen, France. Preliminary simulations indicate that in our experiment the gain of the optimal law is about $15 \%$ with respect to a constant operating temperature.

In order to present our main results we need to introduce precisely our problem.

\subsection{Statement of the problem}

1.1.1. Chemical kinetics. In this article, we shall restrict our study to a batch reactor in which three species $X_{1}, X_{2}, X_{3}$ are reacting according to the scheme $X_{1} \rightarrow X_{2} \rightarrow X_{3}$, where the two reactions are irreversible and of the $n_{i}$-th order with respect to the species $X_{i}, i=1,2$. Assuming that the reactions are at constant volume, denote by $c_{j}, 1 \leq j \leq 3$ the molar concentration of $X_{j}$ and by $T$ the temperature of the reactions; from the laws of chemical kinetics [7] we know that they satisfy:

$$
\left\{\begin{array}{l}
\dot{c}_{1}=-k_{1} c_{1}^{n_{1}} \\
\dot{c}_{2}=k_{1} c_{1}^{n_{1}}-k_{2} c_{2}^{n_{2}} \\
\dot{c}_{3}=k_{2} c_{2}^{n_{2}}
\end{array}\right.
$$

where $\dot{c}$ denotes $\frac{d}{d t}(c)$

The parameters $k_{i}$ are depending on $T$ according to Arrhenius law

$$
k_{i}=A_{i} \exp \left(-E_{i} / R T\right) \quad, \quad i=1,2
$$

where $A_{i}, E_{i}$ are respectively the frequency factor and the activation energy of the $i$-th reaction and $R$ is the gas constant; all these parameters are positive. Similar equations can be obtained when dealing with a network of two simple reactions $n_{1} X_{1} \rightarrow n_{2} X_{2} \rightarrow n_{3} X_{3}$, where the $n_{j}$ 's are the stoichiometric coefficients of the reactions.

Our optimal control problem is the following: minimize the batch time when a desired production quantity is fixed for a batch. The desired product can be $z=c_{2} / c_{1}$ (problems of index 1 ) or $c_{2}$ (problems of index 2 ). Hence we shall solve time minimal problems with final constraint $z\left(t_{F}\right)=d_{1}$ (resp. $\left.c_{2}\left(t_{F}\right)=d_{2}\right)$, where $t_{F}$ is the batch time, and with initial condition $z(0)<d_{1}$ (resp. $\left.c_{2}(0)<d_{2}\right)$.

ESAIM: COCV, DECEMBER 1998, VOL. 3, 407-467 


\subsubsection{Controls. Two mathematical controls are possible:}

$T$, subject to constraints $T_{m} \leq T \leq T_{M}$; the associated problems are $\mathcal{P}_{1}$ (target $\left.z=d_{1}\right)$ and $\mathcal{P}_{2}\left(\right.$ target $\left.c_{2}=d_{2}\right)$

or

$u=\dot{T}$, subject to constraints $u_{-} \leq u \leq u_{+}$with $u_{-}<0<u_{+}$; the associated problems are $\dot{\mathcal{P}}_{1}\left(\right.$ target $\left.z=d_{1}\right)$ and $\dot{\mathcal{P}}_{2}$ (target $c_{2}=d_{2}$ ).

- The choice of the temperature as control is clearly related to the chemical process. If we introduce

$$
v=k_{1}, \quad \alpha=E_{2} / E_{1}, \quad \beta=A_{2} / A_{1}^{\alpha}
$$

then system (1.1) becomes:

$$
\left\{\begin{array}{l}
\dot{c}_{1}=-v c_{1}^{n_{1}} \\
\dot{c}_{2}=v c_{1}^{n_{1}}-\beta v^{\alpha} c_{2}^{n_{2}} \\
\dot{c}_{3}=\beta v^{\alpha} c_{2}^{n_{2}}
\end{array}\right.
$$

and $v$ can be taken as the control, since $v$ is an increasing one-to-one function of $T$, the constraints $T_{m} \leq T \leq T_{M}$ being equivalent to $v_{m} \leq v \leq v_{M}$. Observe that system (1.4) is affine with respect to $v$ if and only if $\alpha=1$; in this particular case the ratio $d c_{2} / d c_{1}$ does not depend on $v$ and the system is not controllable. Since in general the system is not affine with respect to the input it may happen that there is no admissible time optimal control law; this is due to a relaxation phenomenon analyzed in [6].

- If we cannot directly track the optimal temperature profile in the previous system (1.4), we choose $u=\dot{T}$ as the control and we get the following system (which is affine with respect to $u$ ):

$$
\left\{\begin{array}{l}
\dot{c}_{1}=-v c_{1}^{n_{1}} \\
\dot{c}_{2}=v c_{1}^{n_{1}}-\beta v^{\alpha} c_{2}^{n_{2}} \\
\dot{c}_{3}=\beta v^{\alpha} c_{2}^{n_{2}} \\
\dot{v}=h(v) u \text { with } h(v)=\left(R v / E_{1}\right) \ln ^{2}\left(v / A_{1}\right)
\end{array}\right.
$$

By considering $u=\dot{T}$ as a control law, we compute an optimal law related only to the chemical network; this choice is an idealization and the temperature can become negative. To handle this difficulty we must introduce constraint on the state- coordinate $v$; this problem will not be considered in our study.

1.1.3. Physical space. The physical space $P$ of our problem is defined as follows:

$$
\left\{\begin{array}{l}
c_{1}>0, c_{2} \geq 0\left[c_{2}(t)>0 \text { if } t \text { is not the initial time }\right], c_{3} \geq 0 \\
z<d_{1} \text { or } c_{2}<d_{2} \text { except at final time, } \\
0<v<A_{1} \text { since } v=k_{1}=A_{1} \exp \left(-E_{1} / R T\right) \text { with } T>0 .
\end{array}\right.
$$

Then the following inequality:

$$
h(v)>0
$$

comes from (1.5) and (1.6). 
1.1.4. An EXAMPLE. The main properties of our study can be well understood by considering the following example. Notice that according to system (1.1), $z=c_{2} / c_{1}$ satisfies:

$$
\dot{z}=(v+v z) c_{1}^{n_{1}-1}-\beta v^{\alpha} z^{n_{2}} c_{1}^{n_{2}-1}
$$

Therefore $\dot{z}$ is independant of $c_{1}$ if and only if $n_{1}=n_{2}=1$. In this case $n_{1}=$ $n_{2}=1$ the structure of $\mathcal{P}_{1}$ and $\dot{\mathcal{P}}_{1}$ is particular and allows simplifications. This introduces the lightning analysis of the next section.

\subsection{Solving problem $\mathcal{P}_{1}$ in the case $n_{1}=n_{2}=1$}

1.2.1. Computations. According to section 1.1, problem $\mathcal{P}_{1}$ is the time minimal problem of reaching the target $\left(z=d_{1}\right)$ from an initial value $z_{0} \in$ $\left[0, d_{1}\right.$ [ of $z$ for the dynamical system (1.4) which becomes in the coordinates $c_{1}, z, c_{3}$ when $n_{1}=n_{2}=1$ :

$$
\left\{\begin{array}{l}
\dot{c}_{1}=-v c_{1} \\
\dot{z}=v+v z-\beta v^{\alpha} z \\
\dot{c}_{3}=\beta v^{\alpha} c_{2}
\end{array}\right.
$$

the variable $v$ such that $v_{m} \leq v \leq v_{M}$ being considered as the control.

Note that the optimal law is: maximize $\dot{z}$ subject to $v_{m} \leq v \leq v_{M}$. Note also that the target $\left(z=d_{1}\right)$ is not accessible from $z_{0} \in\left[0, d_{1}\right.$ [ such that $\max _{v, t} \dot{z} \leq 0$, where $\max _{v, t} \dot{z}$ is the maximum of $\dot{z}$ for any $v \in\left[v_{m}, v_{M}\right]$ and for any time t.

Consider then $H\left(c_{1}, z, v\right)=\dot{z}$. From (1.8) it comes

$$
\begin{aligned}
& H=0 \text { when } v=0 \\
& \frac{\partial H}{\partial v}=1+z-\alpha \beta v^{\alpha-1} z \\
& \frac{\partial^{2} H}{\partial v^{2}}=-\alpha(\alpha-1) \beta v^{\alpha-2} z
\end{aligned}
$$

So if $\alpha \neq 1, H$ is a non-linear function of $v$ such that there exists a single positive value $\hat{v}$ of $v$ satisfying $\frac{\partial H}{\partial v}=0$ and so corresponding to an extremum of $H$, which is from (1.11):

- a maximum (positive since (1.9) holds) if $\alpha>1$,

- a minimum (negative since (1.9) holds) if $\alpha<1$.

Let us introduce $G$ (resp. $S$ ) the set of points $(v>0, z>0)$ such that $H=0$ (resp. $\left.\frac{\partial H}{\partial v}=0\right)$. From (1.8), $G$ is defined by $z\left(\beta v^{\alpha-1}-1\right)=1$, and from (1.10), $S$ is defined by $z\left(\alpha \beta v^{\alpha-1}-1\right)=1$ : so if $\alpha \neq 1, G \cap S=\emptyset$.

1.2.2. Results. Figure 1 shows $G$ and $S$ in the cases $\alpha<1, \alpha>1$ and a careful observation of this figure gives the optimal trajectories. In order to describe these optimal trajectories, let us denote by $\gamma_{m}$ (resp. $\left.\gamma_{M}, \hat{\gamma}\right)$ any arc satisfying (1.8) with control $v_{m}$ (resp. $v_{M}, \hat{v}$ ). Then, for $\gamma_{a}, \gamma_{b}, \gamma_{c}$ in $\left\{\gamma_{m}, \gamma_{M}, \hat{\gamma}\right\}, \gamma_{a} \gamma_{b} \gamma_{c}$ denotes the concatenation of a $\gamma_{a}$ arc followed by a $\gamma_{b}$ arc and then ended by a $\gamma_{c}$ arc where each arc of the sequence may be empty.

ESAIM: COCV, DECEMBER 1998, VoL. 3, 407-467 


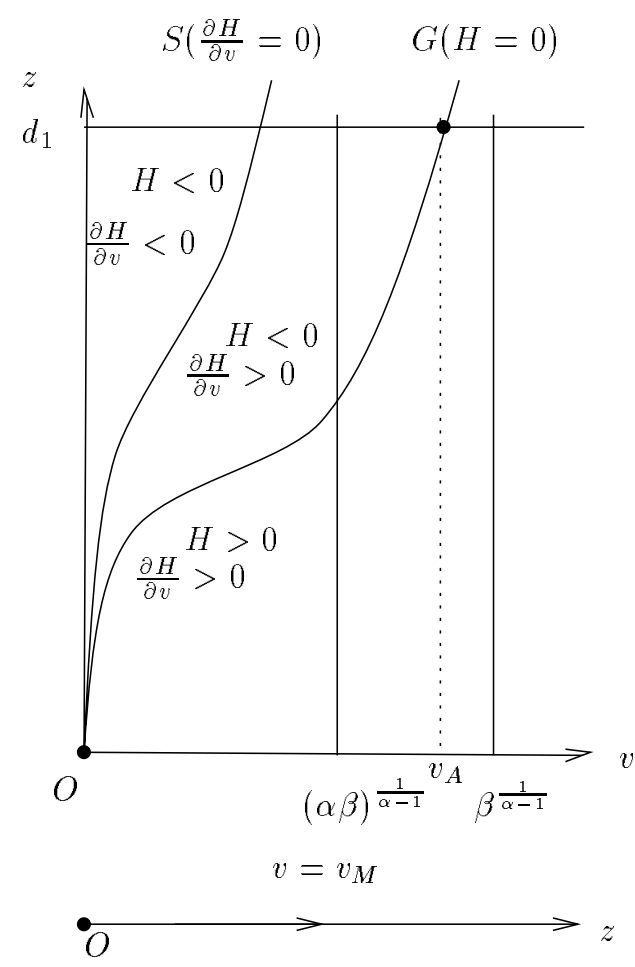

Figure 1 (i) : $\alpha<1$

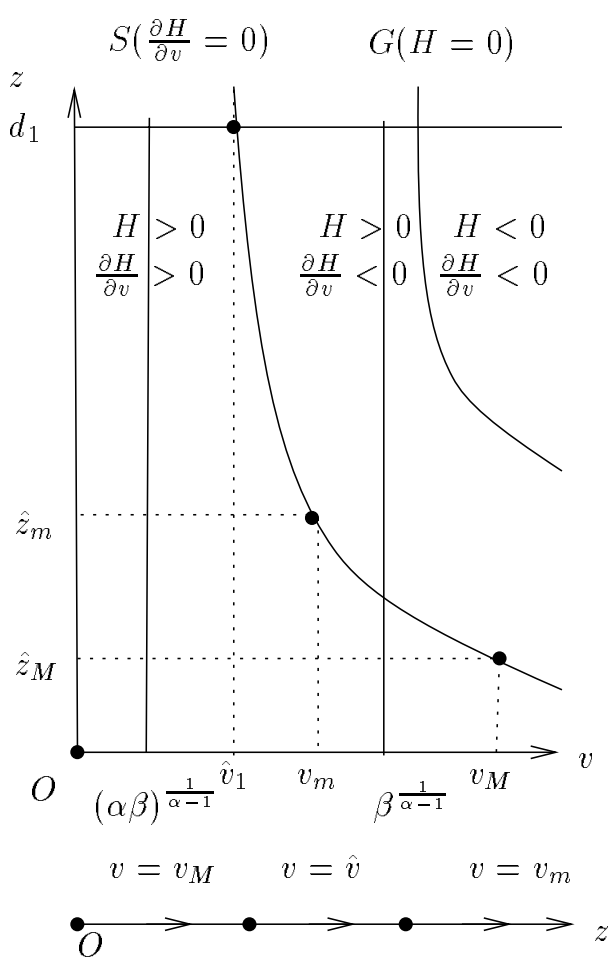

Figure 1 (ii) : $\alpha>1$

Figure 1

CASE $\alpha<1$. For any $z_{0}$ in $\left[0, d_{1}[\right.$,

- if $v_{M}<v_{A}$, where $v_{A}=\left(\frac{\beta d_{1}}{1+d_{1}}\right)^{\frac{1}{1-\alpha}}$ is the $v$-coordinate of $G \cap(z=$ $\left.d_{1}\right)$, the target $\left(z=d_{1}\right)$ is not accessible,

- if $v_{M} \geq v_{A}$, optimal trajectories are $\gamma_{M}$.

CASE $\alpha>1$. For any $z_{0}$ in $\left[0, d_{1}[\right.$,

- if $v_{M} \leq \hat{v}_{1}$, where $\hat{v}_{1}=\left(\frac{\alpha \beta d_{1}}{1+d_{1}}\right)^{\frac{1}{1-\alpha}}$ is the $v$-coordinate of $S \cap\left(z=d_{1}\right)$, optimal trajectories are $\gamma_{M}$,

- if $v_{m} \leq \hat{v}_{1}<v_{M}$, optimal trajectories are $\gamma_{M} \hat{\gamma}, \gamma_{M}$ being empty if and only if $z_{0}>\hat{z}_{M}$ where $\hat{z}_{M}=1 /\left(\alpha \beta v_{M}^{\alpha-1}-1\right)$ is the $z$-coordinate of $S \cap\left(v=v_{M}\right)$,

- if $\hat{v}_{1}<v_{m}<\beta^{\frac{1}{1-\alpha}}$, optimal trajectories are $\gamma_{M} \hat{\gamma} \gamma_{m}, \gamma_{M}$ being empty if and only if $z_{0}>\hat{z}_{M}$, and $\gamma_{M} \hat{\gamma}$ being empty if and only if $z_{0}>\hat{z}_{m}$ where $\hat{z}_{m}=1 /\left(\alpha \beta v_{m}^{\alpha-1}-1\right)$ is the $z$-coordinate of $S \cap\left(v=v_{m}\right)$,

- if $v_{m} \geq \beta^{\frac{1}{1-\alpha}}$, the target $\left(z=d_{1}\right)$ is not accessible.

1.2.3. Conclusion. This example shows the following phenomenon. The value $\alpha=1$ is a bifurcation value for the parameter $\alpha$ which is the ratio of activation energies $E_{1} / E_{2}$ (cf. (1.3) in section 1.1.2); the optimal law appears to be radically different in the case $\alpha<1$ and $\alpha>1$. More precisely, the optimal policy when the target is accessible is: 
- when $\alpha<1$, to apply the maximal temperature $T_{M}$

- whereas if $\alpha>1$, there exists an intermediate optimal heat law in some domain of the state space which is neither $T_{m}$ nor $T_{M}$. Such a law is called a singular optimal control, see [3]; it plays an important role in our problem; it is related to the convexity of the function $v \mapsto H=\dot{z}$ and all the analysis relies on the behavior of $H$.

\subsection{Maximum Principle and Extremals}

In order to make this article self-contained and to give the results as clearly as possible, let us recall some basic concepts and results about Pontryagin's Maximum Principle (in brief PMP) and extremals.

1.3.1. Statement of PMP. Consider a system of the form

$$
\dot{x}(t)=f(x(t), u(t)), x(t) \in \mathbb{R}^{n}, u(t) \in \mathbb{R}^{m}
$$

where $f$ is an analytic mapping from $\mathbb{R}^{n} \times \mathbb{R}$ to $\mathbb{R}^{n}$ and where the set $\mathcal{U}$ of admissible controls is the set of measurable mappings $u(\cdot)$ defined on an interval $\left[t_{0}(u), 0\right]$ of $\mathbb{R}^{-}$and taking their values in $\left[u_{1}, u_{2}\right]$. Let $N$ be a regular analytic submanifold of $\mathbb{R}^{n}$. The PMP asserts that if $u^{*}(t), t \in\left[t_{0}^{*}, 0\right]$ is an optimal control for the time minimal control problem with terminal manifold $N$, then there exists a so-called adjoint vector $p^{*}(t) \in \mathbb{R}^{n} \backslash\left\{0_{\mathbb{R}^{n}}\right\}$ with $p^{*}$ absolutely continuous, such that the following equations are satisfied almost everywhere on $\left[t_{0}^{*}, 0\right]$ :

$$
\begin{aligned}
& \frac{d}{d t}\left(x^{*}\right)=\frac{\partial H}{\partial p}\left(x^{*}, p^{*}, u^{*}\right), \frac{d}{d t}\left(p^{*}\right)=-\frac{\partial H}{\partial x}\left(x^{*}, p^{*}, u^{*}\right) \\
& H\left(x^{*}, p^{*}, u^{*}\right)=M\left(x^{*}, p^{*}\right)
\end{aligned}
$$

where $H(x, p, u)=\langle p, f(x, u)\rangle,\langle\cdot, \cdot\rangle$ being the canonical inner product in $\mathbb{R}^{n}$ and where $M(x, p)=\max _{u \in\left[u_{1}, u_{2}\right]} H(x, p, u)$. Moreover we have:

$$
t \mapsto M\left(x^{*}(t), p^{*}(t)\right) \text { is constant and non-negative }
$$

and at the final time 0 the so-called transversality conditions are satisfied:

$$
x^{*}(0) \in N, p^{*}(0) \text { is orthogonal to } T_{x^{*}(0)} N
$$

where $T_{x} N$ denotes the tangent space to $N$ at $x$.

\subsubsection{Definitions.}

- System (1.13) is called the hamiltonian lift of (1.12), and $H$ is called the Hamiltonian.

- Any $(x, p, u)$ solution to (1.13), (1.14) and (1.15) is called an extremal (sometimes the adjoint state $p$ will be omitted).

- Any extremal $(x, p, u)$ that satisfies the transversality conditions (1.16) is called a BC-extremal.

- Any extremal $(x, p, u)$ such that $M(x, p)=0$ is called exceptional.

- An extremal $(x, p, u)$ is called regular if and only if for almost all $t$, $u(t)$ is equal to $u_{1}$ or to $u_{2}$; if moreover $u$ is piecewise constant, the extremal is called bang-bang.

- An extremal $(x, p, u)$ is called singular if and only if for every time $\frac{\partial H}{\partial u}(x, p, u)=0$ 
- Let $(x, p, u)$ be an extremal. A time $\tau$ is called a switching time if and only if $\tau$ belongs to the closure of the set of times where $x$ is not $\mathcal{C}^{1}$; the corresponding point $x(\tau)$ is then called a switching point.

1.3.3. Notation. As in section 1.2.2, if $\gamma_{a}$ and $\gamma_{b}$ are two arcs solutions to system (1.12), $\gamma_{a} \gamma_{b}$ denotes the concatenation of $\gamma_{a}$ followed by $\gamma_{b}$ (this corresponds to the concatenation of the controls associated to $\gamma_{a}$ and then to $\left.\gamma_{b}\right)$.

1.3.4. Singular extremals. By definition a singular extremal $(x, p, u)$ is a solution to $(1.13),(1.14)$ and $(1.15)$ satisfying $\frac{\partial H}{\partial u}=0$; from (1.14) it has to satisfy the Legendre condition $\left.\frac{\partial^{2} H}{\partial u^{2}}\right|_{(x, p, u)} \leq 0$; if this inequality is strict for every time $t$, it is called the strong Legendre condition. Then by the implicit function theorem the singular control can be locally computed as a function of $(x, p)$ by solving $\frac{\partial H}{\partial u}=0$; equivalently the singular control can be obtained as solution to the Cauchy problem for the differential equation $\frac{d}{d t} \frac{\partial H}{\partial u}=0$, the strong Legendre condition implying the existence of such a solution.

Recall that, in order to be admissible, the singular control has to belong to $\left[u_{1}, u_{2}\right]$. Any point of a singular extremal where the singular control is equal to $u_{1}$ or to $u_{2}$ is called a saturation point.

\subsection{Statement of the main Results}

We shall give the main results of our article. To simplify the presentation we shall assume that each reaction is of first order, i.e. that $n_{1}=n_{2}=1$.

1.4.1. Problem $\dot{\mathcal{P}}_{1}$ IN The CASE $n_{1}=n_{2}=1$. According to section $1.1, \dot{\mathcal{P}}_{1}$ is the time minimal control problem of reaching the target $\left(z=d_{1}\right)$ from an initial value $z_{0} \in\left[0, d_{1}\right.$ [ of $z$ for the dynamical system (1.5) and for control $u$ such that $u_{-} \leq u \leq u_{+}$with $u_{-}<0<u_{+}$. When $n_{1}=n_{2}=1$, system (1.5) becomes in the state-coordinates $c_{1}, z, c_{3}, v$ :

$$
\left\{\begin{array}{l}
\dot{c}_{1}=-v c_{1} \\
\dot{z}=v+v z-\beta v^{\alpha} z \\
\dot{c}_{3}=\beta v^{\alpha} c_{2} \\
\dot{v}=h(v) u \text { with } h(v)=\left(R v / E_{1}\right) \ln ^{2}\left(v / A_{1}\right)
\end{array}\right.
$$

We shall prove subsequently (cf. section 3.1 ) that we can restrict our study to the planar system:

$$
\left\{\begin{array}{l}
\dot{z}=v+v z-\beta v^{\alpha} z \\
\dot{v}=h(v) u \text { with } h(v)=\left(R v / E_{1}\right) \ln ^{2}\left(v / A_{1}\right)
\end{array}\right.
$$

Let us discuss the corresponding optimal synthesis.

We introduce the following notations:

Let $u_{s}$ be the singular control, and let us denote by $\gamma_{-}$(resp. $\gamma_{+}, \gamma_{s}$ ) any arc satisfying (1.18) with control $u_{-}$(resp. $u_{+}, u_{s}$ ).

Let $A$ be the set of the points of the target $\left(z=d_{1}\right)$ that are accessible from 
$\left(z<d_{1}\right)$.

Let $G$ be the set of the points $(z>0, v \in] 0, A_{1}[)$ such that $\dot{z}=0$.

Let $S$ be the set of the points $(z>0, v \in] 0, A_{1}[)$ such that $\frac{d}{d t} \frac{\partial H}{\partial u}=0$, where $H$ denotes the Hamiltonian corresponding to system (1.18) and let $S_{1}=S \cap N$. From section 1.3.4, $S$ contains all the singular extremals. Computations show that the singular control $u_{s}$ is negative and that there is on $S$ at most one saturation point $S_{\text {sat }}$, where $u_{s}=u_{-}$.

In the following figures (Figures 2 and 3 ) we assume that $v_{A}<A_{1}$, where $v_{A}$ denotes the v-coordinate of $G \cap\left(z=d_{1}\right)$; if $A_{1} \leq v_{A}$, these figures have to be restricted to $\left(v<A_{1}\right)$.

CASE $\alpha<1$. Near the target, every optimal trajectory is of the form $\gamma_{+}$ and the local synthesis is given by Figure 2 .

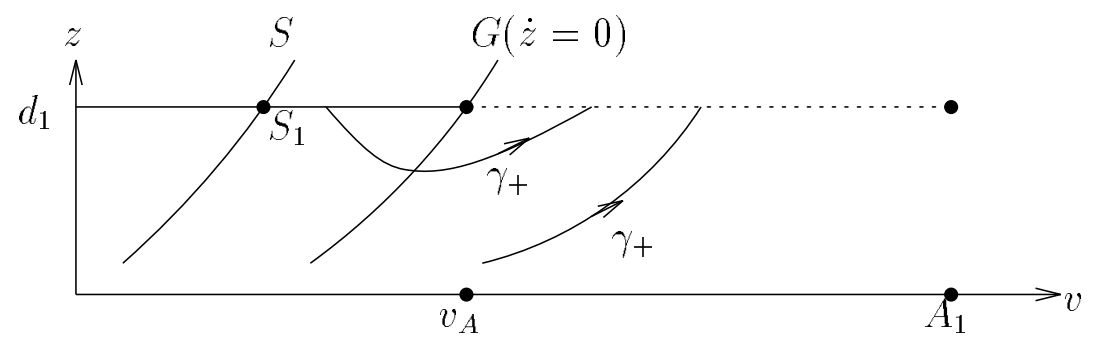

A, empty if and only if $A_{1} \leq v_{A}$

Figure 2

CASE $\alpha>1$. In this case we can describe the global synthesis. Let $z_{\text {sat }}$ denote the $z$-coordinate of the saturation point $S_{\mathrm{sat}}$. We have two situations: - if $z_{\text {sat }} \geq d_{1}$, optimal trajectories are $\gamma_{+} \gamma_{-}$and the synthesis is represented on Figure 3(i),

- if $z_{\text {sat }}<d_{1}$, optimal trajectories are $\gamma_{+} \gamma_{-} \gamma_{s}$ and the synthesis is given by Figure 3(ii)

where each arc of these sequences may be empty

1.4.2. Problem $\mathcal{P}_{2}$ IN The CASE $n_{1}=n_{2}=1$. According to section 1.1, $\mathcal{P}_{2}$ is the time minimal problem of reaching the target $\left(c_{2}=d_{2}\right)$ from an initial value $\left(c_{2}\right)_{0} \in\left[0, d_{2}\right.$ [ of $c_{2}$ for the system (1.4) where the control is $v$ such that $v_{m} \leq v \leq v_{M}$.

We shall prove subsequently (cf. section 3.1 ) that we can restrict our study to the planar system:

$$
\left\{\begin{array}{l}
\dot{c}_{1}=-v c_{1} \\
\dot{c}_{2}=v c_{1}-\beta v^{\alpha} c_{2}
\end{array}\right.
$$

Let us denote by $\hat{v}$ the singular control and by $\gamma_{m}$ (resp. $\gamma_{M}, \hat{\gamma}$ ) any arc satisfying (1.19) with control $v_{m}$ (resp. $\left.v_{M}, \hat{v}\right)$.

Let $A$ be the set of the points of the target $\left(c_{2}=d_{2}\right)$ that are accessible from $\left(c_{2}<d_{2}\right)$.

Let $E_{m}$ (resp. $E_{M}$ ) be the point of target such that $\dot{c}_{2}=0$ when $v=v_{m}$ (resp. $v=v_{M}$ ).

ESAIM: COCV, DECEMBER 1998, VoL. 3, 407-467 


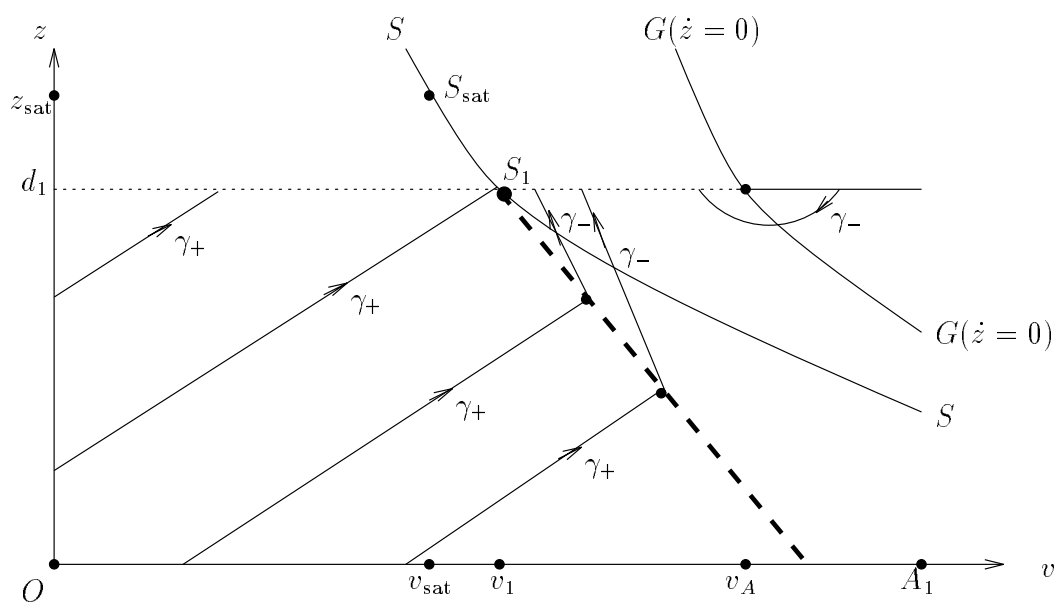

Figure 3(i) : $z_{\text {sat }} \geq d_{1}$

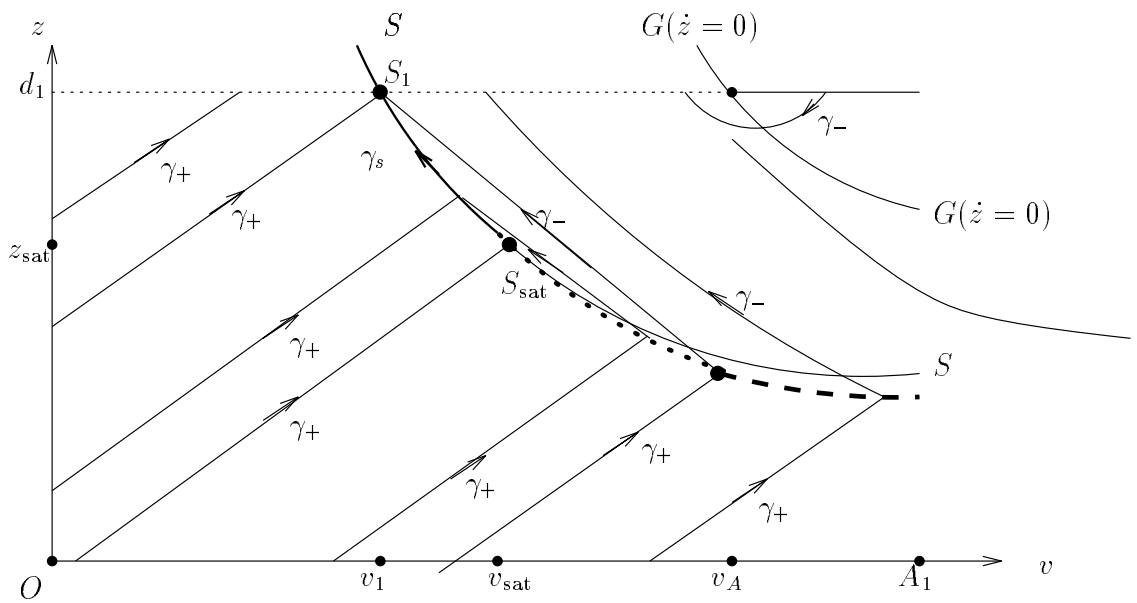

Figure 3(ii) : $z_{\text {sat }}<d_{1}$

A, _ - - set of switching points for $\gamma_{+} \gamma_{-}$,

.... set of first switching points for $\gamma_{+} \gamma_{-} \gamma_{s}$

Figure 3

Let $S_{m}$ (resp. $S_{M}$ ) be the point of the target such that $0=\left.\frac{\partial H}{\partial v}\right|_{v=v_{m}}$, i.e. $v_{m}=\left.\hat{v}\right|_{t=0}\left(\operatorname{resp} .0=\left.\frac{\partial H}{\partial v}\right|_{v=v_{M}}\right.$, i.e. $\left.v_{M}=\left.\hat{v}\right|_{t=0}\right)$ where $H$ is the Hamiltonian (recall that, from section 1.3.4, the singular control $\hat{v}$ is solution to $\left.\frac{\partial H}{\partial v}=0\right)$. 
CASE $\alpha<1$. Each optimal trajectory is of the form $\gamma_{M}$ and the synthesis is represented on Figure 4.

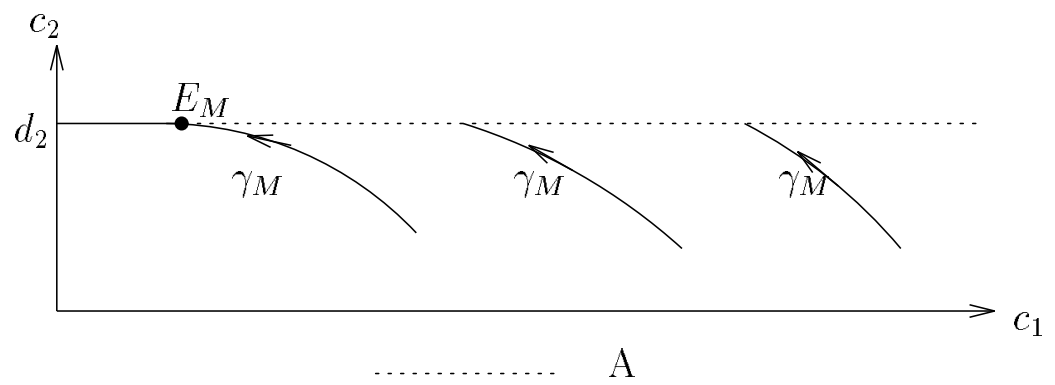

Figure 4

CASE $\alpha>1$. Results are threefold:

- Near the target, each optimal policy has at most one switching.

- Each optimal trajectory is of the form $\gamma_{M} \hat{\gamma} \gamma_{m}$, where each arc of the sequence may be empty, and each open-loop control is $\mathcal{C}^{0}$.

- Numerical simulations (cf. section 8) show that the optimal synthesis is given by Figure 5 .

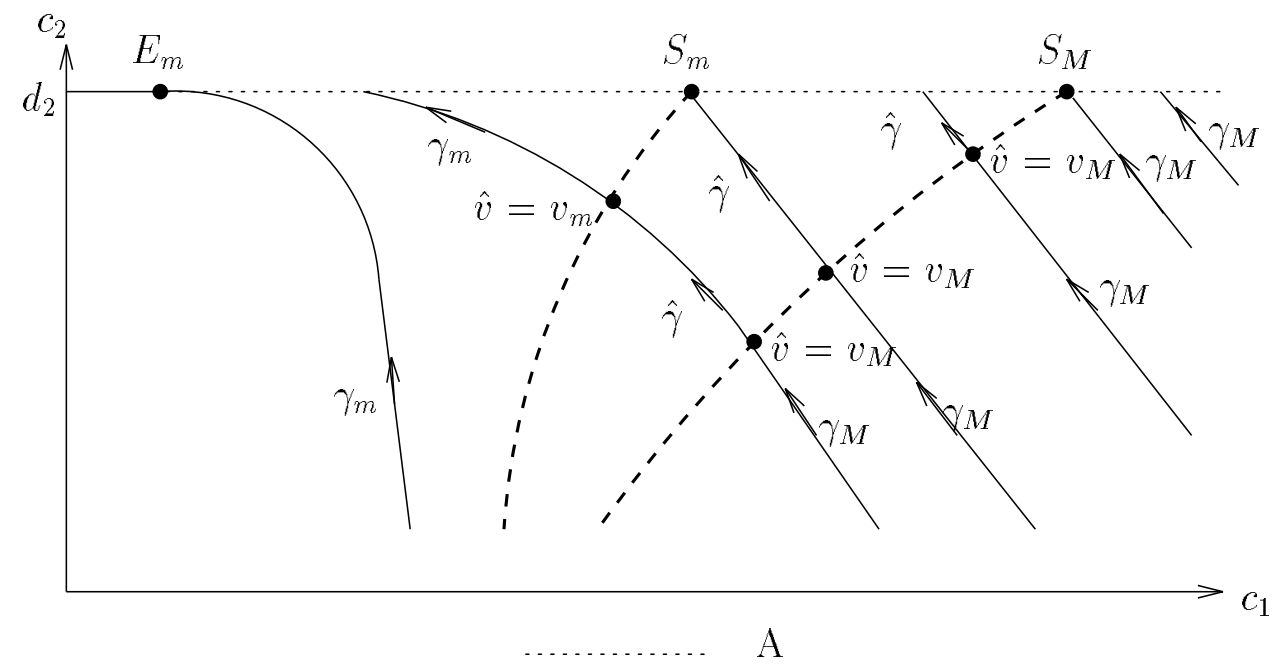

Figure 5

1.4.3. Problem $\dot{\mathcal{P}}_{2}$ IN The CASE $n_{1}=n_{2}=1$. According to section $1.1, \dot{\mathcal{P}}_{2}$ is the time minimal problem of reaching the target $\left(c_{2}=d_{2}\right)$ from an initial value $\left(c_{2}\right)_{0} \in\left[0, d_{2}\right.$ [ of $c_{2}$ for the control system (1.5) where the control $u$ satisfies $u_{-} \leq u \leq u_{+}$with $u_{-}<0<u_{+}$.

We shall prove subsequently (cf. section 3.1 ) that we can restrict our study to the three dimensional system:

$$
\left\{\begin{array}{l}
\dot{c}_{1}=-v c_{1} \\
\dot{c}_{2}=v c_{1}-\beta v^{\alpha} c_{2} \\
\dot{v}=h(v) u \text { with } h(v)=\left(R v / E_{1}\right) \ln ^{2}\left(v / A_{1}\right)
\end{array}\right.
$$


Let us denote by $u_{s}$ the singular control and by $\gamma_{-}$(resp. $\gamma_{+}, \gamma_{s}$ ) any arc satisfying (1.20) with control $u_{-}$(resp. $\left.u_{+}, u_{s}\right)$.

The set $A$ of the points of the target $\left(c_{2}=d_{2}\right)$ that are accessible from $\left(c_{2}<d_{2}\right)$ is included in the set $\mathcal{A}$ of the points of the target such that $\dot{c}_{2} \geq 0$, the frontier of which is the set $\mathcal{E}$ of the points of the target such that $\dot{c}_{2}=0$.

Let $S_{2}$ be the set of the points of the target such that $\frac{d}{d t} \frac{\partial H}{\partial u}=0$, where $H$ denotes the Hamiltonian (from section 1.3.4 any singular extremal satisfies this condition). It turns out that at the final time the singular control is negative and that there is on $S_{2}$ one saturation point $S_{\text {sat }}$, where $u_{-}=\left.u_{s}\right|_{t=0}$.

On Figure 6 we describe the stratification of the target by the optimal control in the $\left(v, c_{1}\right)$ coordinates; it is deduced from the equations of the Maximum Principle, except near the set $\mathcal{E}$ where the situation is intricated because of accessibility problems.

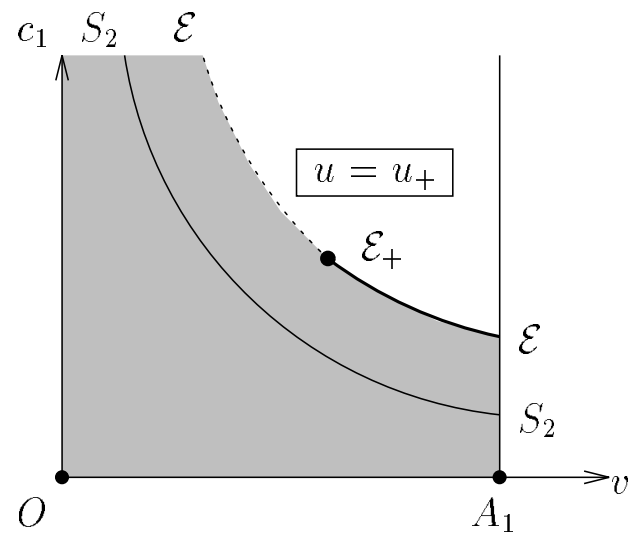

Figure 6 (i) : $\alpha<1$

non-accessible points:

and

on $\mathcal{E}$, with $\mathcal{E}_{+}$non-accessible

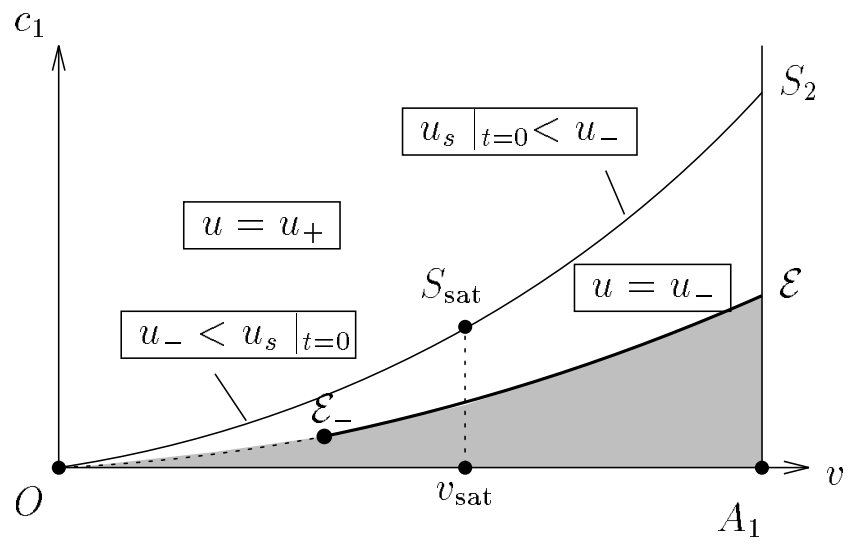

Figure 6 (ii) : $\alpha>1$

non-accessible points:

and

on $\mathcal{E}$, with $\mathcal{E}_{-}$accessible

Figure 6 : stratification of the target 
CASE $\alpha<1$. It turns out that near the target the optimal policy is $u=u_{+}$. Moreover there exists one point $\mathcal{E}_{+}$on $\mathcal{E}$ such that $\frac{d^{2}}{d t^{2}}\left(c_{2}\right)=0$; near this point the local synthesis is given by Figure 7

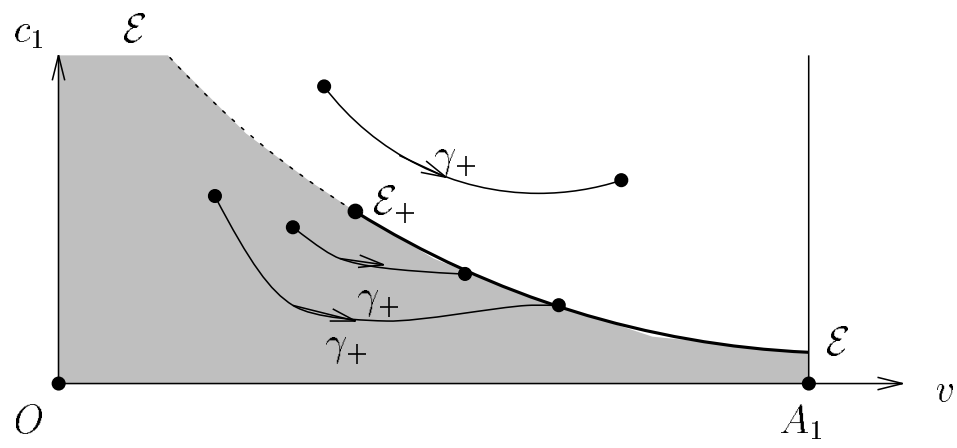

non-accessible points:

and

on $\mathcal{E}$, with $\mathcal{E}_{+}$non-accessible

Figure 7 : local synthesis near $\mathcal{E}_{+}$when $\alpha<1$

CASE $\alpha>1$. We got the following local and global results:

Local synthesis Near the target the optimal synthesis is described as follows:

- For optimal trajectories arriving near $S_{2}$, the synthesis can be topologically described by an invariant foliation $\left(v=v_{0}\right)$. Let $v_{\text {sat }}$ denote the $v$-coordinate of the saturation point $S_{\text {sat }}$; the syntheses in each leaf $\left(v=v_{0}\right)$ are shown by Figure 8:

if $v_{0}<v_{\text {sat }}$, optimal trajectories are of the form $\gamma_{+} \gamma_{-} \gamma_{s}$ (see Figure 8 (i))

if $v_{0} \geq v_{\text {sat }}$, optimal trajectories are of the form $\gamma_{+} \gamma_{-}$(see Figure 8 (ii)) where each arc of these sequences may be empty.

- For optimal trajectories arriving near $\mathcal{E}$, the situation is intricated. Near the target, close enough to $\mathcal{E}$, the optimal policy is $u=u_{-}$. Moreover there exists one point $\mathcal{E}_{-}$on $\mathcal{E}$ where $\frac{d^{2}}{d t^{2}}\left(c_{2}\right)=0$; let $v_{-}$denote the $v$-coordinate of $\mathcal{E}_{-}$. Near a point $\mathcal{E}_{0} \neq \mathcal{E}_{-}$of $\mathcal{E}$, the optimal synthesis is described by a $\mathcal{C}^{0}$ invariant foliation $\mathcal{F}:\left(v=v_{0}\right)$ the leaves of which are given by Figures 9 (i) and (ii); near $\mathcal{E}_{-}$there is no such foliation and the synthesis is given by Figure 9 (iii).

Global switching rules Each optimal control law has at most two switchings and each optimal trajectory is of the form $\gamma_{+} \gamma_{-} \gamma_{s}$, where each arc of this sequence may be empty.

\subsection{Summary}

This paper is organized as follows. In section 2 we recall extremality results for time minimal problems with single in put affine systems. In section 3 we introduce the concepts of projected and reduced problems, which are straightforward but important tools in our study. The use of projected problems is related to symmetry properties of our systems. The concept of ESAIM: CoCv, DeCEMBer 1998, VoL. 3, 407-467 


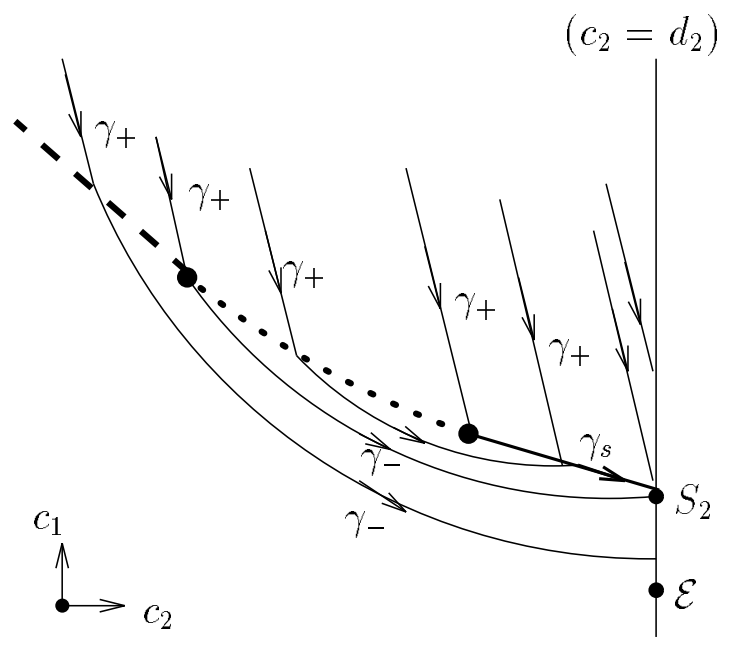

Figure 8 (i) $: v_{0}<v_{\text {sat }}$

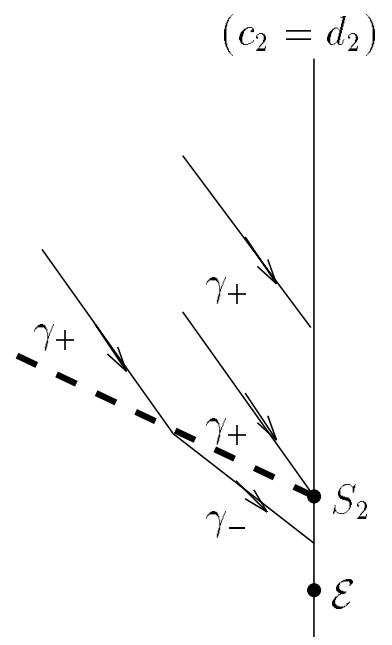

Figure 8 (ii) $: v_{0} \geq v_{\text {sat }}$

$$
\begin{gathered}
-\ldots \ldots \text { set of switching points for } \gamma_{+} \gamma_{-} \\
\ldots . \quad \text { set of first switching points for } \gamma_{+} \gamma_{-} \gamma_{s}
\end{gathered}
$$

Figure 8 : local synthesis near $S_{2}$ in the leaf $v=v_{0}$ when $\alpha>1$

reduced problem is more acute; it is related to Goh's transformation and lightens the relationship between problems $\mathcal{P}_{i}$ (with control $T$ ) and $\dot{\mathcal{P}}_{i}$ (with control $\dot{T}$ ), $i=1,2$. In section 4 we analyze problem $\mathcal{P}_{2}$; first we solve $\mathcal{P}_{2}$ near the target when $\alpha \neq 1$; secondly we find global bounds on the number of switchings. In section 5 (resp. 6) we apply results of [5] and [14] to compute in the case $\alpha \neq 1$ the closed-loop optimal control near the target for problem $\dot{\mathcal{P}}_{1}$ when $n_{1}=n_{2}=1$ (resp. problem $\dot{\mathcal{P}}_{2}$ for any $n_{1}, n_{2}$ ); secondly in the case $\alpha>1$, we find global switching rules for $\dot{\mathcal{P}}_{1}$ when $n_{1}=n_{2}=1$ (resp. $\dot{\mathcal{P}}_{2}$, except when $n_{2}=1$ and $n_{1} \geq 2$ ). Section 7 deals with the concept of conjugate and focal points. Endly, section 8 is an appendix regrouping commented numerical experiments.

\section{EXTREMALS FOR TIME MINIMAL PROBLEMS WITH SINGLE INPUT AFFINE SYSTEMS}

\subsection{Definitions and elementary Results}

2.1.1. Statement of the problem. Throughout this section 2, the considered system is:

$$
\dot{x}(t)=X(x(t))+u(t) Y(x(t)) \quad, \quad x(t) \in \mathbb{R}^{n} \text { and } u(t) \in \mathbb{R}
$$

where $X$ and $Y$ are analytic vector fields. As in section 1.3.1, the set $\mathcal{U}$ of admissible controls is the set of measurable mappings $u(\cdot)$ defined on an interval $\left[t_{0}(u), 0\right]$ of $\mathbb{R}^{-}$and taking their values in $\left[u_{1}, u_{2}\right]$; and again $N$ denotes a regular analytic submanifold of $\mathbb{R}^{n}$, which is the target to be reached within minimal time.

This problem is said to be flat if and only if $Y$ is everywhere tangent to $N$. Note that problems $\dot{\mathcal{P}}_{1}$ and $\dot{\mathcal{P}}_{2}$ defined in section 1.1 match the above 


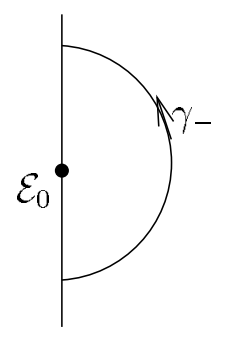

Figure 9 (i) $: v_{0}<v_{-}$

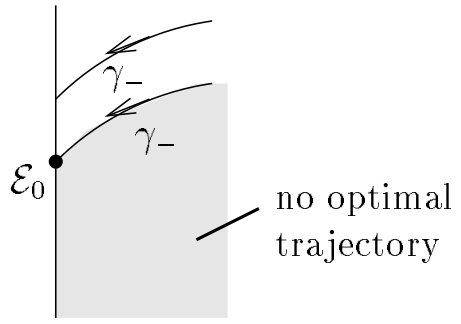

Figure 9 (ii): $v_{0}>v_{-}$

local synthesis near $\mathcal{E}_{0}=\mathcal{E} \cap\left(v=v_{0}\right)$ in the leaf $v=v_{0}$ when $\alpha>1$

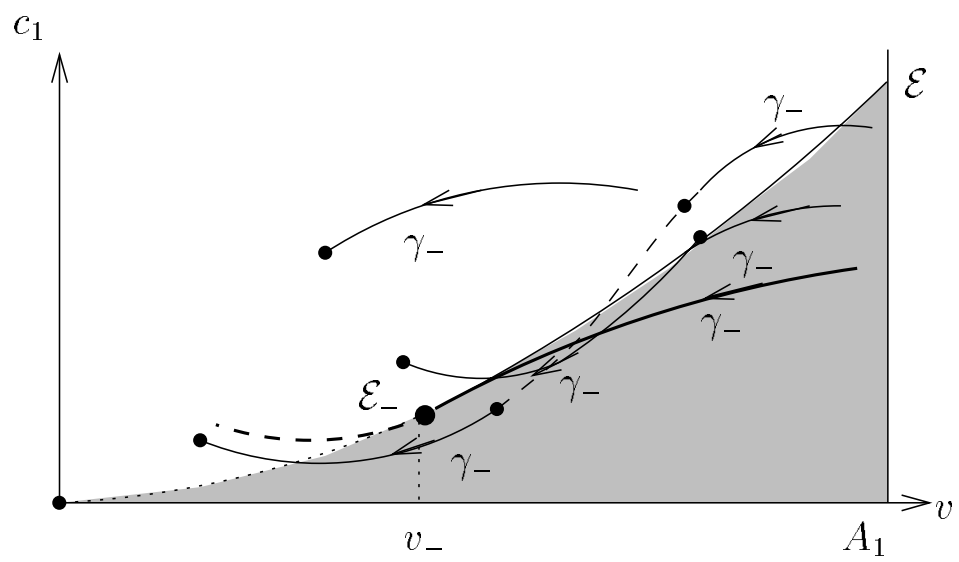

non-accessible points: and ... on $\mathcal{E}$, with $\mathcal{E}_{-}$accessible

Figure 9 (iii): local synthesis near $\mathcal{E}_{-}$when $\alpha>1$

Figure 9

conditions and are both flat.

Notations Let us denote by $u_{s}$ the singular control and by $\gamma_{1}$ (resp. $\gamma_{2}, \gamma_{s}$ ) any arc satisfying (2.1) with control $u_{1}$ (resp. $u_{2}, u_{s}$ ).

2.1.2. Switching function. For any extremal $(x, p, u)$ the Hamiltonian is $H(x, p, u)=\langle p, X(x)+u Y(x)\rangle$ (from section 1.3.1 applied to system (2.1)). Hence the equation $\frac{\partial H}{\partial u}=0$ (which is satisfied by any singular extremal) is equivalent to $\langle p, Y(x)\rangle=0$. The mapping:

$$
\Phi: t \mapsto\langle p(t), Y(x(t))\rangle
$$

evaluated along $(x, p)$ is called the switching function.

Computing we get:

$$
\dot{\Phi}(t)=\langle p(t),[X, Y](x(t))\rangle
$$

and along any smooth extremal:

$$
\ddot{\Phi}(t)=\left\langle p(t), \operatorname{ad}^{2} X(Y)(x(t))-u(t) \operatorname{ad}^{2} Y(X)(x(t))\right\rangle
$$


where the Lie bracket of two analytic vector fields $Z_{1}, Z_{2}$ is computed with the convention: $\left[Z_{1}, Z_{2}\right](x)=\frac{\partial Z_{2}}{\partial x}(x) Z_{1}(x)-\frac{\partial Z_{1}}{\partial x}(x) Z_{2}(x)$, and where $\operatorname{ad} Z_{1}\left(Z_{2}\right)$ is the mapping defined by $\operatorname{ad} Z_{1}\left(Z_{2}\right)=\left[Z_{1}, Z_{2}\right]$.

\subsubsection{Elementary Regular cases.}

Proposition 2.1. Let $z_{0}=\left(x_{0}, p_{0}\right) \in \mathbb{R}^{n} \times\left(\mathbb{R}^{n} \backslash\left\{0_{\mathbb{R}^{n}}\right\}\right)$ be such that $\left\langle p_{0}, Y\left(x_{0}\right)\right\rangle \neq 0$. In a neighborhood of $z_{0}$, any extremal is a $\gamma_{1}$ (resp. $\gamma_{2}$ ) arc if and only if $\left\langle p_{0}, Y\left(x_{0}\right)\right\rangle$ is negative (resp. positive).

Proof. From PMP any extremal maximizes the Hamiltonian.

Corollary 2.2. Let $(x, p, u)$ be an extremal with switching time $\tau$; then necessarily $\langle p(\tau), Y(x(\tau))\rangle=0$, i.e. $\Phi(\tau)=0$.

Proposition 2.3. Let $z_{0}=\left(x_{0}, p_{0}\right) \in \mathbb{R}^{n} \times\left(\mathbb{R}^{n} \backslash\left\{0_{\mathbb{R}^{n}}\right\}\right)$ be such that $Y\left(x_{0}\right) \neq$ $0,\left\langle p_{0}, Y\left(x_{0}\right)\right\rangle=0$ and $\left\langle p_{0},[X, Y]\left(x_{0}\right)\right\rangle \neq 0$ : such a point $z_{0}$ is called $a$ normal switching point. Then close enough to $z_{0}$ there is only one extremal containing $z_{0}$, which is $\gamma_{2} \gamma_{1}$ (resp. $\gamma_{1} \gamma_{2}$ ) if and only if $\left\langle p_{0},[X, Y]\left(x_{0}\right)\right\rangle$ is negative (resp. positive), $x_{0}$ being the switching point.

Proof. Consider $(x, p, u)$ an extremal that contains $z_{0}$, and let $\tau_{0}$ be the time such that $z_{0}=\left(x\left(\tau_{0}\right), p\left(\tau_{0}\right)\right)$. Since $\left\langle p_{0},[X, Y]\left(x_{0}\right)\right\rangle \neq 0$ i.e. (from (2.3)) $\dot{\Phi}\left(\tau_{0}\right) \neq 0$, close enough to $z_{0}$ the considered extremal has no singular arc (indeed, from section 2.1.2, any singular arc satisfies $\Phi \equiv 0$ ). Then the result comes from the maximization of the Hamiltonian (cf. PMP) after expanding $\Phi$ at $\tau_{0}$ up to the first order.

\subsubsection{Singular extremals: Generalities.}

Definitions and notations. A point $(x, p)$ is called ordinary if and only if $\left\langle p, \operatorname{ad}^{2} Y(X)(x)\right\rangle \neq 0$; let $\theta$ be the set of non-ordinary points. A singular extremal $(x, p, u)$ such that $(x(t), p(t)) \in \mathbb{R}^{2 n} \backslash \theta$ is said to be of order 2 . Let $\Sigma$ be the variety $\left\{(x, p) \in \mathbb{R}^{n} \times\left(\mathbb{R}^{n} \backslash\left\{0_{\mathbb{R}^{n}}\right\}\right) ;\langle p, Y(x)\rangle=0\right\}$ and let $\Sigma^{\prime}=\left\{(x, p) \in \mathbb{R}^{n} \times\left(\mathbb{R}^{n} \backslash\left\{0_{\mathbb{R}^{n}}\right\}\right) ;\langle p, Y(x)\rangle=\langle p,[X, Y](x)\rangle=0\right\}$ included in $\Sigma$.

Let $H_{s}$ be the restriction to $\Sigma^{\prime} \backslash \theta$ of the mapping: $(x, p) \mapsto\langle p, X(x)+$ $\left.u_{s}(x, p) Y(x)\right\rangle$, where:

$$
u_{s}(x, p)=\frac{\left\langle p, \operatorname{ad}^{2} X(Y)(x)\right\rangle}{\left\langle p, \operatorname{ad}^{2} Y(X)(x)\right\rangle}
$$

Proposition 2.4. Any singular extremal $(x, p, u)$ is such that $(x(t), p(t)) \in$ $\Sigma^{\prime}$. Moreover the singular extremals $(x, p, u)$ of order 2 are defined by:

$$
u(t)=u_{s}(x(t), p(t)) \text { where } u_{s}(x, p) \text { is defined in }(2.5),
$$

and $(x, p)$ is a solution to the ordinary differential analytic system:

$$
\frac{d x}{d t}=\frac{\partial H_{s}}{\partial p}(x, p) \text { and } \frac{d p}{d t}=-\frac{\partial H_{s}}{\partial x}(x, p)
$$

with the following admissibility constraints:

$$
u_{1} \leq u_{s}(x, p) \leq u_{2}
$$

Proof. See [4]. 
Definitions. Let $(x, p, u)$ be a singular extremal of order 2 and let $h$ be the value of the Hamiltonian (non-negative constant from PMP) along this extremal.

The considered extremal is exceptional if and only if $h=0$ (cf. section 1.3.2).

If $h>0$, the considered extremal is called hyperbolic (resp. elliptic) if and only if $\left\langle p(t), \operatorname{ad}^{2} Y(X)(x(t))\right\rangle$ is negative (resp. positive).

\subsection{Optimality of Singular extremals For Limit-Problem}

2.2.1. Statement of Limit-Problem. Limit-problem associated to the problem stated in section 2.1 .1 is a time minimal problem with the same single input affine system (2.1), but where

- the boundary constraints $u \in\left[u_{1}, u_{2}\right]$ on the control are relaxed and we admit a specific class of impulse controls. More precisely a trajectory is a finite concatenation of arcs corresponding to bounded measurable controls and finite jumps in the $Y$ direction; it is associated to a set of admissible controls which will be denoted by $\mathcal{U}^{\prime}$,

and where

- the terminal manifold is a point.

The optimality status of singular extremals of order 2 for such limit-problems was studied in [4] under the following assumptions:

2.2.2. Assumptions. Consider $(x, p, u)$ a singular extremal defined on $\left[t_{0}, 0\right]$ and assume that:

$\left(H_{0}^{\ell}\right) t \mapsto x(t)$ is one-to-one.

Since the concept of singular extremal is feedback-invariant (see [4]) one may set $u \equiv 0$. Let us then introduce $K(t)=\operatorname{Span}\left\{\operatorname{ad}^{k} X(Y)(x(t)) ; k \in \mathbb{N}\right\}$. Since $(x, p, u)$ is a singular extremal, it is known ([10]) that the codimension of $K(t)$ is not zero. Moreover let us then assume that:

$\left(H_{1}^{\ell}\right) \forall t \in\left[t_{0}, 0\right], K(t)$ is of codimension one, and $K(t)$ is spanned by the vectors $\operatorname{ad}^{k} X(Y)(x(t)), k=0, \ldots, n-2$.

$\left(H_{2}^{\ell}\right) \forall t \in\left[t_{0}, 0\right], \operatorname{ad}^{2} Y(X)(x(t)) \notin K(t)$.

$\left(H_{3}^{\ell}\right)$ If $n=2, \forall t \in\left[t_{0}, 0\right] X(x(t))$ and $Y(x(t))$ are linearly independent.

If $n \geq 3, \forall t \in\left[t_{0}, 0\right] X(x(t)) \notin \operatorname{Span}\left\{\operatorname{ad}^{k} X(Y)(x(t)) ; k=0, \ldots, n-3\right\}$.

\subsubsection{Results.}

TheOREM 2.5. Let $(x, p, u)$ be a singular extremal defined on $\left[t_{0}, 0\right]$ and satisfying $\left(H_{0}^{\ell}\right)-\left(H_{3}^{\ell}\right)$. Note that the adjoint vector $p$ is then unique up to a non-zero factor, and that for any $t$ in $\left[t_{0}, 0\right], p(t)$ is orthogonal to $K(t)$; so $(x(t), p(t)) \in \Sigma^{\prime} \backslash \theta$ and thus $(x, p, u)$ is of order 2. Then there exists a $\mathcal{C}^{0}$ neighborhood $U$ of $x$ such that $x$ is a time-minimizing (resp. maximizing) trajectory with respect to all the solutions to (2.1) contained in $U$ and joining $x\left(t_{0}\right)$ to $x(0)$ [the set of admissible controls being $\mathcal{U}^{\prime}$ defined in section 2.2.1] if $(x, p, u)$ is exceptional or hyperbolic (resp. elliptic) and if $t_{1 c}<0$, where $t_{1 c}$ is the first conjugate time to $t_{0}$ along $x$. Note that if $(x, p, u)$ is hyperbolic and if $t_{1 c}>0$, the trajectory is not even $\mathcal{C}^{1}$-time-minimizing.

Proof. See [4].

ESAIM: CoCV, DECEMBER 1998, VoL. 3, 407-467 
This theorem solves the time minimality problem for singular trajectories satisfying $\left(H_{0}^{\ell}\right)-\left(H_{3}^{\ell}\right)$ when any bounded measurable control is admissible and when the terminal manifold is a point; it has to be adapted to deal with problems $\dot{\mathcal{P}}_{1}$ and $\dot{\mathcal{P}}_{2}$ defined in section 1.1 where the control is subject to boundary constraints and where the terminal manifold is of codimension one.

\subsection{Fold CASE}

Consider $z_{0}=\left(x_{0}, p_{0}\right) \in \Sigma^{\prime}$ (defined in section 2.1.4) such that:

$$
\left\{\begin{array}{l}
Y\left(x_{0}\right),[X, Y]\left(x_{0}\right) \text { are linearly independent, } \\
\text { for } \mathrm{i} \in\{1,2\}, \lambda_{i}=\left\langle p_{0}, \operatorname{ad}^{2} X(Y)\left(x_{0}\right)-u_{i} \operatorname{ad}^{2} Y(X)\left(x_{0}\right)\right\rangle \text { is not zero }
\end{array}\right.
$$

Such a point $z_{0}$ is called a fold point; and from [12] and [4] the extremals near $z_{0}$ for the problem defined in section 2.1 are of the form:

- if $\lambda_{1} \lambda_{2}>0$ (parabolic case): $\gamma_{1} \gamma_{2} \gamma_{1}$ or $\gamma_{2} \gamma_{1} \gamma_{2}$

- if $\lambda_{1}<0$ and $\lambda_{2}>0$ (hyperbolic case): $\gamma_{i} \gamma_{s} \gamma_{j}$ with $(i, j) \in\{1,2\}^{2}$

- if $\lambda_{1}>0$ and $\lambda_{2}<0$ (elliptic case): bang-bang extremals, with no uniform bounds on the number of switchings on any compact containing $z_{0}$, but with a finite number of switchings for any extremal

where each arc of these sequences may be empty.

Proof. The behavior of regular extremals near $z_{0}$ has been classified in [12] and is shown by Figure 10 , where $z_{i}$ denotes $z=(x, p)$ for control $u_{i}$, $i=1,2$.

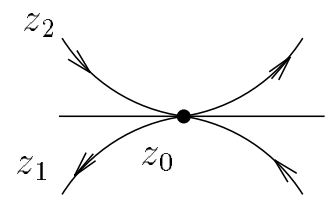

hyperbolic case

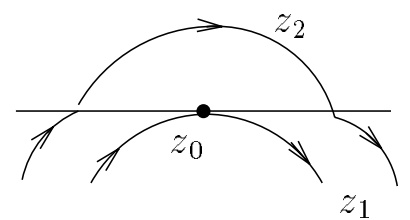

parabolic case

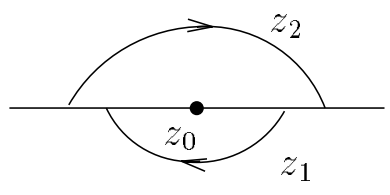

elliptic case

Figure 10

In the parabolic case, elementary computations show that $u_{s} \notin\left[u_{1}, u_{2}\right]$ and so $u_{s}$ is not admissible. Moreover, if we assume that the singular extremal arc passing through $z_{0}$ satisfies assumptions $\left(H_{0}^{\ell}\right)-\left(H_{3}^{\ell}\right)$ (defined in section 2.2.2) we know from [4] that it is fast (resp. slow) for limit-problem in the hyperbolic (resp. elliptic) case.

\subsection{Singular extremals in the Case $n=3$}

Considering the problem defined in section 2.1 when $n=3$, let us define: $D=-\operatorname{det}\left(Y,[X, Y], \operatorname{ad}^{2} Y(X)\right), D^{\prime}=\operatorname{det}\left(Y,[X, Y], \operatorname{ad}^{2} X(Y)\right)$ and $D^{\prime \prime}=$ $\operatorname{det}(Y,[X, Y], X)$.

The singular extremals $(x, p, u)$ satisfying $D \neq 0$ are clearly of order 2 ; moreover $x$ satisfies:

$$
\dot{x}=X(x)+u_{s}(x) Y(x) \text { with } u_{s}=-\frac{D^{\prime}}{D}
$$


Proof. Since $\Phi=\langle p, Y(x)\rangle$ is zero at any time along any singular extremal, (2.3) and (2.4) (cf. section 2.1.2) imply $\langle p,[X, Y](x)\rangle \equiv 0$ and $\left\langle p, \operatorname{ad}^{2} X(Y)(x)-u \operatorname{ad}^{2} Y(X)(x)\right\rangle \equiv 0$; so we get $D^{\prime}+u D \equiv 0$ since $p$ is never zero.

Corollary 2.6. The singular extremals included in $\mathbb{R}^{3} \backslash\{D=0\}$ satisfy $\left(H_{1}^{\ell}\right)-\left(H_{3}^{\ell}\right)$ (defined in section 2.2.2); they are hyperbolic (resp. elliptic) if and only if $D^{\prime \prime} D>0$ (resp. $D^{\prime \prime} D<0$ ); they are exceptional if and only if $D^{\prime \prime}=0$.

\section{Projected problem. Reduced problem}

\subsection{Projected problem}

3.1.1. Statement of the Problems. Every object is supposed to be real analytic. Let $M$ and $M^{\prime}$ be two manifolds, let $\pi$ denote a submersion from $M$ into $M^{\prime}$. Let $N$ (resp. $N^{\prime}$ ) be a regular submanifold of $M$ (resp. $M^{\prime}$ ). Consider a system of the form:

$$
\dot{x}(t)=f(x(t), u(t)) \quad, \quad x(t) \in M \text { and } u(t) \in \Omega \subset \mathbb{R}
$$

Let us assume that:

(i) for each admissible control $u(\cdot)$, the differential system (3.1) is complete,

(ii) for each fixed $u$ in $\Omega$, the differential system (3.1) can be $\pi$-projected on $M^{\prime}$

(iii) $N=\pi^{-1}\left(N^{\prime}\right)$.

Note that when considering local coordinates, assumptions (ii) and (iii) mean that there exist coordinates $x=\left(x^{\prime}, x^{\prime \prime}\right)$ on $M$ such that $\pi$ is the projection $M \longrightarrow M^{\prime}, N$ being identified to the set of all $\left(N^{\prime}, x^{\prime \prime}\right)$. and $\left(x^{\prime}, x^{\prime \prime}\right) \mapsto x^{\prime}$

such that (3.1) can be written as:

$$
\dot{x}^{\prime}=f^{\prime}\left(x^{\prime}, u\right) \text { and } \dot{x}^{\prime \prime}=f^{\prime \prime}(x, u)
$$

Let us denote by $\mathcal{P}$ (resp. $\mathcal{P}^{\prime}$ ) the time minimal problem associated to the system $\dot{x}=f(x, u)$ (resp. $\dot{x}^{\prime}=f^{\prime}\left(x^{\prime}, u\right)$ ) with terminal manifold $N$ (resp. $\left.N^{\prime}\right): \mathcal{P}^{\prime}$ is called the projected problem associated to $\mathcal{P}$. Then let us denote by $x\left(t, x_{0}, u\right)$ the solution to $\dot{x}=f(x, u)$ initiating at initial time $t_{0}$ from $x_{0} \in M$, and by $x^{\prime}\left(t, x_{0}^{\prime}, u\right)$ the solution to $\dot{x}^{\prime}=f^{\prime}\left(x^{\prime}, u\right)$ initiating at initial time $t_{0}$ from $x_{0}^{\prime}=\pi\left(x_{0}\right) \in M^{\prime}$.

\subsubsection{Results FOR The PROBlems STATED IN SECTION 3.1.1.}

Lemma 3.1. The trajectory $x\left(t, x_{0}, u\right)$ defined on $\left[t_{0}(u), 0\right]$ is a solution to $\mathcal{P}$ if and only if $x^{\prime}\left(t, x_{0}^{\prime}, u\right)$ is a solution to $\mathcal{P}^{\prime}$ on $\left[t_{0}(u), 0\right]$.

Proof. From assumptions (i) and (ii) in section 3.1.1, one has at any time $t$ such that the right hand member of (3.1) is defined:

$$
x^{\prime}\left(t, x_{0}^{\prime}, u\right)=\pi\left(x\left(t, x_{0}, u\right)\right)
$$

Moreover $N=\pi^{-1}\left(N^{\prime}\right)$ from (iii) in section 3.1.1. 
Lemma 3.2. Every $B C$-extremal $\left(x^{\prime}, p^{\prime}, u\right)$ of the projected problem $\mathcal{P}^{\prime}$ can be lifted into a $B C$-extremal $(x, p, u)$ of the original problem $\mathcal{P}$ with $x=\left(x^{\prime}, x^{\prime \prime}\right)$ and $p=\left(p^{\prime}, 0\right)$.

Proof. We know from section 3.1.1 that system (3.1) can be locally written as:

$$
\dot{x}^{\prime}=f^{\prime}\left(x^{\prime}, u\right) \text { and } \dot{x}^{\prime \prime}=f^{\prime \prime}(x, u) \text { with } x=\left(x^{\prime}, x^{\prime \prime}\right) .
$$

Moreover the adjoint vector $p=\left(p^{\prime}, p^{\prime \prime}\right)$ of any extremal $(x, p, u)$ of $\mathcal{P}$ satisfies:

$$
\dot{p}^{\prime}=-p^{\prime} \frac{\partial f^{\prime}}{\partial x^{\prime}}\left(x^{\prime}, u\right)-p^{\prime \prime} \frac{\partial f^{\prime \prime}}{\partial x^{\prime}}\left(x^{\prime}, x^{\prime \prime}, u\right) \text { and } \dot{p}^{\prime \prime}=-p^{\prime \prime} \frac{\partial f^{\prime \prime}}{\partial x^{\prime \prime}}\left(x^{\prime}, x^{\prime \prime}, u\right) .
$$

Since any extremal $\left(x^{\prime}, p^{\prime}, u\right)$ of $\mathcal{P}^{\prime}$ satisfies:

$$
\dot{x}^{\prime}=f^{\prime}\left(x^{\prime}, u\right) \text { and } \dot{p}^{\prime}=-p^{\prime} \frac{\partial f^{\prime}}{\partial x^{\prime}}\left(x^{\prime}, u\right),
$$

it can be lifted into $\left(x=\left(x^{\prime}, x^{\prime \prime}\right), p=\left(p^{\prime}, 0\right), u\right)$ [where $x^{\prime \prime}$ is any solution to $\left.\dot{x}^{\prime \prime}=f^{\prime \prime}\left(x^{\prime}, x^{\prime \prime}, u\right)\right]$ which is an extremal of $\mathcal{P}$.

Moreover for both extremals $\left(x^{\prime}, p^{\prime}, u\right)$ of $\mathcal{P}^{\prime}$ and $\left(x=\left(x^{\prime}, x^{\prime \prime}\right), p=\left(p^{\prime}, 0\right), u\right)$ of $\mathcal{P}$ the transversality conditions are:

$$
x^{\prime}(0) \in N^{\prime} \text { and } p^{\prime}(0) \text { is orthogonal to } T_{x^{\prime}(0)} N^{\prime}
$$

since $N$ is identified to the set of all $\left(N^{\prime}, x^{\prime \prime}\right)$.

Remark 3.3. Not every extremal of problem $\mathcal{P}$ can be projected onto an extremal of problem $\mathcal{P}^{\prime}$. So, although from Lemma 3.2 problems $\mathcal{P}$ and $\mathcal{P}^{\prime}$ are equivalent, studying the solutions of the PMP for $\mathcal{P}^{\prime}$ is simpler than for $\mathcal{P}$ because there are less extremals.

\subsubsection{Applications.}

- $\dot{\mathcal{P}}_{1}$ in the case $n_{1}=n_{2}=1$ is the time minimal problem associated to the system (1.17) (cf. section 1.4.1) with terminal manifold ( $z=d_{1}$ ). Then from section 3.1 .2 we can consider the equivalent time minimal projected problem $\dot{\mathcal{P}}_{1}^{\prime}$ associated to the system (1.18) (cf. section 1.4.1) with terminal manifold $\left(z=d_{1}\right.$ ) and control $u$ such that $u_{-} \leq u \leq u_{+}$and $u_{-}<0<u_{+}$.

- $\mathcal{P}_{2}$ is, according to section 1.1, the time minimal problem associated to the system (1.4) with terminal manifold $\left(c_{2}=d_{2}\right)$. Consider the adapted coordinate:

$$
x_{1}=\left\{\begin{array}{l}
\ln c_{1} \text { if } n_{1}=1 \\
\frac{1}{1-n_{1}} c_{1}^{1-n_{1}} \text { if } n_{1}>1
\end{array}\right.
$$

System (1.4) becomes in the state-coordinates $x_{1}, c_{2}, c_{3}$ :

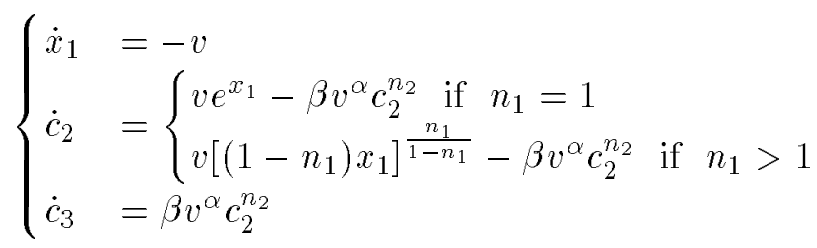


Then from section 3.1.2 we can consider the equivalent time minimal projected problem $\mathcal{P}_{2}^{\prime}$ associated to the system:

$$
\left\{\begin{array}{l}
\dot{x}_{1}=-v \\
\dot{c}_{2}=\left\{\begin{array}{l}
v e^{x_{1}}-\beta v^{\alpha} c_{2}^{n_{2}} \text { if } n_{1}=1 \\
v\left[\left(1-n_{1}\right) x_{1}\right]^{\frac{n_{1}}{1-n_{1}}}-\beta v^{\alpha} c_{2}^{n_{2}} \text { if } n_{1}>1
\end{array}\right.
\end{array}\right.
$$

with terminal manifold $\left(c_{2}=d_{2}\right)$ and control $v$ such that $v_{m} \leq v \leq v_{M}$.

- $\dot{\mathcal{P}}_{2}$ is, according to section 1.1 , the time minimal problem associated to the system (1.5) with terminal manifold $\left(c_{2}=d_{2}\right)$. System (1.5) becomes in the state-coordinates $x_{1}, c_{2}, c_{3}, v$ system (3.3) with the supplementary equation $\dot{v}=h(v) u$. Then from section 3.1.2 we can consider the equivalent time minimal projected problem $\dot{\mathcal{P}}_{2}^{\prime}$ associated to the system:

$$
\left\{\begin{array}{l}
\dot{x}_{1}=-v \\
\dot{c}_{2}=\left\{\begin{array}{l}
v e^{x_{1}}-\beta v^{\alpha} c_{2}^{n_{2}} \text { if } n_{1}=1 \\
v\left[\left(1-n_{1}\right) x_{1}\right]^{\frac{n_{1}}{1-n_{1}}}-\beta v^{\alpha} c_{2}^{n_{2}} \text { if } n_{1}>1 \\
\dot{v}=h(v) u \text { with } h(v)=\left(R v / E_{1}\right) \ln ^{2}\left(v / A_{1}\right)
\end{array}\right.
\end{array}\right.
$$

with terminal manifold $\left(c_{2}=d_{2}\right)$ and control $u$ such that $u_{-} \leq u \leq u_{+}$and $u_{-}<0<u_{+}$.

\subsection{ReducED PROBLEM}

3.2.1. Preliminary. Consider the time minimal problem stated in section 2.1.1 with affine single input system $(2.1)$ in $\mathbb{R}^{n}(n \geq 2)$ and with flatness hypothesis.

For batch reactors:

- it was already noticed in section 2.1.1 that problems $\dot{\mathcal{P}}_{1}$ and $\dot{\mathcal{P}}_{2}$ (with control $\dot{T}$ ) defined in section 1.1 meet all these conditions. Then $T$ (or equivalently $v$ ) is a state variable,

- on the contrary, for problems $\mathcal{P}_{1}$ and $\mathcal{P}_{2}$ (defined also in section 1.1) the dynamical system is not affine with respect to the control $T$ (or equivalently $v$ ) when $\alpha \neq 1$.

The aim of this section 3.2 is to relate singular extremals in both cases. Indeed, a general system of the form: $\dot{x}=f(x, u)$ can be interpreted as an affine system with respect to the new control $\dot{u}, u$ becoming a statevariable. Let us study the converse transformation, which is called Goh's transformation.

\subsubsection{RESULTS FOR LIMIT-PROBLEM.}

Definitions and notations. According to section 2.2.1, consider the limit-problem associated to the problem stated in section 2.1.1 with the same single input affine system $(2.1)$ in $\mathbb{R}^{n}(n \geq 2): \dot{x}=X(x)+u Y(x)$ where the boundary constraints on the control $u$ are relaxed and where the terminal manifold is a point (so the flatness hypothesis does not make sense any more).

ESAIM: CoCV, DeCEMBer 1998, Vol. 3, 407-467 
Take $x_{0} \in \mathbb{R}^{n}$ such that $Y\left(x_{0}\right) \neq 0$. Hence, from the flow-box theorem, there exists an open neighborhood $U$ of $x_{0}$ and local coordinates $\left(x^{1}, \ldots, x^{n}\right)$ in $U$ such that $\left.Y\right|_{U}=\frac{\partial}{\partial x^{n}}$. Introducing $\tilde{x}=\left(x^{1}, \ldots x^{n-1}\right)^{t}$ and $\tilde{X}$ such that:

$$
X(x)=\sum_{i=1}^{n} X^{i}(x) \frac{\partial}{\partial x^{i}}=\tilde{X}(x) \frac{\partial}{\partial \tilde{x}}+X^{n}(x) \frac{\partial}{\partial x^{n}},
$$

the restriction of $\dot{x}=X(x)+u Y(x)$ to $U$ becomes:

$$
\dot{\tilde{x}}=\tilde{X}\left(\tilde{x}, x^{n}\right) \quad, \quad \dot{x}^{n}=X^{n}\left(\tilde{x}, x^{n}\right)+u
$$

Then the system $\dot{\tilde{x}}=\tilde{X}\left(\tilde{x}, x^{n}\right)$ is called a reduced system associated to the original affine system $\dot{x}=X(x)+u Y(x), x^{n}$ being the control variable and $\tilde{x}$ belonging to $\tilde{U}$ open subset of $\mathbb{R}^{n-1}$. Note that such a reduced system is not unique.

For the original (resp. reduced) problem, let $p=\left(p_{1}, \ldots, p_{n}\right)^{t}$ (resp. $\tilde{p}=$ $\left(\tilde{p}_{1}, \ldots, \tilde{p}_{n-1}\right)^{t}$ be the dual variables of the state variables $x$ (resp. $\tilde{x}$ ) and let $H(x, p, u)=\langle p, X(x)+u Y(x)\rangle\left(\operatorname{resp} . \tilde{H}\left(\tilde{x}, \tilde{p}, x^{n}\right)=\left\langle\tilde{p}, \tilde{X}\left(\tilde{x}, x^{n}\right)\right\rangle\right)$ be the Hamiltonian.

LEMMA 3.4. The pair $(x, p)$ is the projection on the space of state and adjoint variables of a solution $(x, p, u)$ to:

$$
\dot{x}=\frac{\partial H}{\partial p}(x, p, u) \quad, \quad \dot{p}=-\frac{\partial H}{\partial x}(x, p, u) \quad, \quad \frac{\partial H}{\partial u}(x, p, u) \equiv 0
$$

if and only if $p_{n} \equiv 0$ and $\left(\tilde{x}, \tilde{p}=\left(p_{1}, \ldots, p_{n-1}\right)^{t}, x^{n}\right)$ is a solution to:

$$
\dot{\tilde{x}}=\frac{\partial \tilde{H}}{\partial \tilde{p}}\left(\tilde{x}, \tilde{p}, x^{n}\right) \quad, \quad \dot{\tilde{p}}=-\frac{\partial \tilde{H}}{\partial \tilde{x}}\left(\tilde{x}, \tilde{p}, x^{n}\right) \quad, \quad \frac{\partial \tilde{H}}{\partial x^{n}}\left(\tilde{x}, \tilde{p}, x^{n}\right) \equiv 0
$$

and then the following relations are satisfied:

$$
\begin{array}{r}
\left.\frac{d}{d t} \frac{\partial H}{\partial u}\right|_{(x, p, u)}=\langle p,[X, Y](x)\rangle=-\left.\frac{\partial \tilde{H}}{\partial x^{n}}\right|_{\left(\tilde{x}, \tilde{p}, x^{n}\right)} \\
\left.\frac{\partial}{\partial u} \frac{d^{2}}{d t^{2}} \frac{\partial H}{\partial u}\right|_{(x, p, u)}=-\left\langle p, a d^{2} Y(X)(x)\right\rangle=-\left.\frac{\partial^{2} \tilde{H}}{\left(\partial x^{n}\right)^{2}}\right|_{\left(\tilde{x}, \tilde{p}, x^{n}\right)}
\end{array}
$$

Proof. System (3.7) can be written as:

$$
\begin{cases}\dot{\tilde{x}}=\tilde{X}\left(\tilde{x}, x^{n}\right), \quad \dot{x}^{n}=X^{n}\left(\tilde{x}, x^{n}\right)+u & \text { [i.e. } \left.\dot{x}=\frac{\partial H}{\partial p}(x, p, u)\right] \\ \forall j \in\{1, \ldots, n\} \dot{p}_{j}=-\sum_{i=1}^{n} p_{i} \frac{\partial}{\partial x^{j}} X^{i}\left(\tilde{x}, x^{n}\right) & \text { [i.e. } \left.\dot{p}=-\frac{\partial H}{\partial x}(x, p, u)\right] \\ p_{n} \equiv 0 & \text { [i.e. } \left.\frac{\partial H}{\partial u}(x, p, u) \equiv 0\right]\end{cases}
$$

which is equivalent, when omitting $u$, to: 
$p_{n} \equiv 0$ and

$$
\left\{\begin{array}{l}
\dot{\tilde{x}}=\tilde{X}\left(\tilde{x}, x^{n}\right), \text { i.e. } \dot{\tilde{x}}=\frac{\partial \tilde{H}}{\partial \tilde{p}}\left(\tilde{x}, \tilde{p}, x^{n}\right) \\
\forall j \in\{1, \ldots, n-1\} \dot{p}_{j}=-\sum_{i=1}^{n-1} p_{i} \frac{\partial}{\partial x^{j}} X^{i}\left(\tilde{x}, x^{n}\right), \text { i.e. } \dot{\tilde{p}}=-\frac{\partial \tilde{H}}{\partial \tilde{x}}\left(\tilde{x}, \tilde{p}, x^{n}\right) \\
\sum_{i=1}^{n-1} p_{i} \frac{\partial}{\partial x^{n}} X^{i}\left(\tilde{x}, x^{n}\right) \equiv 0, \text { i.e. } \frac{\partial \tilde{H}}{\partial x^{n}}\left(\tilde{x}, \tilde{p}, x^{n}\right) \equiv 0 \text { with } \tilde{p}=\left(p_{1}, \ldots, p_{n-1}\right)^{t}
\end{array}\right.
$$

that is $p_{n} \equiv 0$ and (3.8) with $\tilde{p}=\left(p_{1}, \ldots, p_{n-1}\right)^{t}$.

From section 2.1.2 we know that, if $\Phi=\left.\frac{\partial H}{\partial u}\right|_{(x, p, u)}=\langle p, Y(x)\rangle$ denotes the switching function of the affine system $\dot{x}=X(x)+u Y(x)$,

$$
\begin{aligned}
& \dot{\Phi}=\left.\frac{d}{d t} \frac{\partial H}{\partial u}\right|_{(x, p, u)}=\langle p,[X, Y](x)\rangle \quad(c f .(2.3)) \\
& \text { and } \ddot{\Phi}=\left.\frac{d^{2}}{d t^{2}} \frac{\partial H}{\partial u}\right|_{(x, p, u)}=\left\langle p, \operatorname{ad}^{2} X(Y)(x)-u \operatorname{ad}^{2} Y(X)(x)\right\rangle \quad(c f .(2.4)) \\
& \text { so }\left.\frac{\partial}{\partial u} \frac{d^{2}}{d t^{2}} \frac{\partial H}{\partial u}\right|_{(x, p, u)}=-\left\langle p, \operatorname{ad}^{2} Y(X)(x)\right\rangle
\end{aligned}
$$

Moreover (3.7) implies (after computations with $Y=\frac{\partial}{\partial x^{n}}, p_{n} \equiv 0$ and $\left.\tilde{p}=\left(p_{1}, \ldots, p_{n-1}\right)^{t}\right)$ :

$$
\langle p,[X, Y](x)\rangle=-\left.\frac{\partial \tilde{H}}{\partial x^{n}}\right|_{\left(\tilde{x}, \tilde{p}, x^{n}\right)} \quad \text { and }\left\langle p, \operatorname{ad}^{2} Y(X)(x)\right\rangle=-\left.\frac{\partial^{2} \tilde{H}}{\left(\partial x^{n}\right)^{2}}\right|_{\left(\tilde{x}, \tilde{p}, x^{n}\right)},
$$

so (3.9) and (3.10) are proved.

Definition 3.5. A singular extremal $(x, p, u)$ for the original affine system satisfies the Legendre-Clebsch condition if and only if $\frac{\partial}{\partial u} \frac{d^{2}}{d t^{2}} \frac{\partial H}{\partial u} \geq 0$ along $(x, p, u)$.

Corollary 3.6. Relation (3.10) implies that, if $(x, p, u)$ is a singular extremal for the original affine system, the Legenre-Clebsch condition along $(x, p, u)$ is equivalent to the Legendre condition (defined in section 1.3.4) along the associated singular extremal for the reduced system.

LEMMA 3.7 (singular controls). Since the concept of singular extremal is feedback-invariant (see [4]) one may set $X^{n}=0$. Thus the restriction of $\dot{x}=X(x)+u Y(x)$ to $U(c f .(3.6))$ is:

$$
\dot{\tilde{x}}=\tilde{X}\left(\tilde{x}, x^{n}\right) \quad, \quad \dot{x}^{n}=u
$$

Consider $(x, p, u)$ a singular extremal of order 2 for this affine system and assume that:

$$
\frac{\partial}{\partial u} \frac{d^{2}}{d t^{2}} \frac{\partial H}{\partial u}>0 \text { along }(x, p, u)
$$

Then the singular control $u$ is given by (2.5) (cf. section 2.1.4):

$$
u=u_{s}(x, p)=\frac{\left\langle p, a d^{2} X(Y)(x)\right\rangle}{\left\langle p, a d^{2} Y(X)(x)\right\rangle}
$$


Moreover, relation (3.10) implies that (3.12) is equivalent to the strong Legendre condition (defined in section 1.3.4) along the associated singular extremal $\left(\tilde{x}, \tilde{p}, x^{n}\right)$ for the reduced system. Then, as noticed in section 1.3.4, we know from the implicit function theorem that the singular control $x^{n}$ can be locally computed as a function of $(\tilde{x}, \tilde{p})$ by solving $\frac{\partial \tilde{H}}{\partial x^{n}}=0$; equivalently $x^{n}$ can be obtained as solution to the Cauchy problem for $\frac{d}{d t} \frac{\partial \tilde{H}}{\partial x^{n}}=0$, which exists since the strong Legendre condition $\frac{\partial^{2} \tilde{H}}{\left(\partial x^{n}\right)^{2}}<0$ holds along $\left(\tilde{x}, \tilde{p}, x^{n}\right)$.

Finally, it comes from (3.11) that: $\dot{x}^{n}=u$.

Lemma 3.8 (Optimality). Theorem 2.5 gives the optimality status of any singular extremal $(x, p, u)$ satisfying $\left(H_{0}^{\ell}\right)-\left(H_{3}^{\ell}\right)$ for the original affine limitproblem. The associated singular extremal $\left(\tilde{x}, \tilde{p}, x^{n}\right)$ for the reduced problem considered in the previous lemma 3.7 has the same optimality status when changing $\mathcal{C}^{0}$-optimality of $(x, p, u)$ into $\mathcal{C}^{1}$-optimality of $\left(\tilde{x}, \tilde{p}, x^{n}\right)$.

Proof. See [4]. Indeed, if $x=\left(\tilde{x}, x^{n}\right)$ and $x^{\prime}=\left(\tilde{x}^{\prime}, x^{m}\right)$ are in a $\mathcal{C}^{0}$ neighborhood, then $x^{n}$ and $x^{\prime n}$ are in a $\mathcal{C}^{0}$-neighborhood; and $x^{n}, x^{m}$ are the control variables for the considered reduced systems.

\subsubsection{Results For flat minimal-time problem with a target of CODIMENSION ONE IN DIMENSION GREATER THAN TWO.}

Statement of the Problem. According to the preliminary 3.2.1 let us consider the time minimal problem stated in section 2.1.1 with:

- an affine single-input system (2.1): $\dot{x}=X(x)+u Y(x)$ in $\mathbb{R}^{n}(n \geq 3)$, the boundary constraints on the control $u$ being relaxed

- a target $N$ which is a regular analytic submanifold of $\mathbb{R}^{n}$ of codimension one

- $Y$ everywhere tangent to $N$ (flatness hypothesis).

This problem is denoted by $\mathcal{P}_{\text {aff }}$.

Let $\vec{n}$ be a normal vector to $N$ pointing outwards the half-space where the considered trajectories lie. Let $S_{N}$ be the subset of $N$ defined by

$$
S_{N}=\{x \in N ;\langle\vec{n},[X, Y](x)\rangle=0\}
$$

Assumptions. Take a point $x_{0}$ of $S_{N}$ as the origin and assume that:

$\left(H_{0}^{r}\right) Y(0) \neq 0$.

Hence, from the flow-box theorem, there exists an open neighborhood $U$ of 0 in $\mathbb{R}^{n}$ and local coordinates $\left(x^{1}, \ldots x^{n}\right)$ in $U$ such that $\left.Y\right|_{U}=\frac{\partial}{\partial x^{n}}$. Let us introduce $\tilde{x}=\left(x^{1}, \ldots, x^{n-1}\right)^{t}$ and $\tilde{\tilde{x}}=\left(x^{2}, \ldots, x^{n-1}\right)^{t}$

$\left(H_{1}^{r}\right) \quad N$ is identified to $\left(x^{1}=0\right)$.

Then $S_{N}=\left\{\left(0, \tilde{\tilde{x}}, x^{n}\right) \in N ; \frac{\partial X^{1}}{\partial x^{n}}\left(0, \tilde{\tilde{x}}, x^{n}\right)=0\right\}$, so $S_{N}$ is defined on $N$ by an equation $\varphi\left(\tilde{\tilde{x}}, x^{n}\right)=0$ with $\varphi\left(0_{\mathbb{R}^{n-1}}\right)=0$ since $0_{\mathbb{R}^{n}} \in S_{N}$

$\left(H_{2}^{r}\right) Y(0)$ is not included in $S_{N}$. 
This implies that $\varphi\left(0_{\mathbb{R}^{n-2}}, x^{n}\right) \neq 0$. So, considering the equation $\varphi(\tilde{\tilde{Z}}, W)=$ 0 for $\tilde{\tilde{Z}}$ in $\mathbb{C}^{n-2}$ and $W$ in $\mathbb{C}$, we know that $W$ is of finite order $k \in \mathbb{N}^{*}$ at $0_{\mathbb{C}^{n-1}}$ i.e. that there exists an analytic function $\varphi_{1}$ such that $\varphi\left(0_{\mathbb{C}^{n-2}}, W\right)=$ $W^{k} \varphi_{1}(W)$ with $\varphi_{1}(0) \neq 0$. Then from the Weierstrass Preparation Theorem there exist analytic functions $a_{1}, \ldots, a_{k}, \varphi_{2}$ defined on a neighborhood $U^{\prime}$ of $0_{\mathbb{C}^{n-1}}$ in $\mathbb{C}^{n-1}$ such that $\varphi(\tilde{\tilde{Z}}, W)=P(\tilde{\tilde{Z}}, W) \varphi_{2}(\tilde{\tilde{Z}}, W)$ with $\varphi_{2}$ never vanishing in $U^{\prime}$ and $P(\tilde{\tilde{Z}}, W)=W^{k}+\sum_{i=1}^{k} a_{i}(\tilde{\tilde{Z}}) W^{k-i}$. So on $U^{\prime}, \varphi(\tilde{\tilde{Z}}, W)=0$ is equivalent to $P(\tilde{\tilde{Z}}, W)=0$ which is polynomial in $W$.

$\left(H_{3}^{r}\right)$ For any $\left(\tilde{\tilde{Z}}_{0}, W\right)$ in $U^{\prime}$ where $\tilde{\tilde{Z}}_{0} \neq 0_{\mathbb{C}^{n-2}}$ is fixed, there are $k$ distinct solutions to the polynomial equation in $W: P\left(\tilde{\tilde{Z}}_{0}, W\right)=0$.

So the restriction of $\tilde{\pi}:(\tilde{\tilde{Z}}, W) \mapsto \tilde{\tilde{Z}}$ to $\left\{(\tilde{\tilde{Z}}, W) \in U^{\prime} ; P(\tilde{\tilde{Z}}, W)=0\right\}$ is a covering map (with branch-point $0_{\mathbb{C}^{n-2}}$ ) which is $k$-sheeted above $\tilde{\tilde{Z}} \neq 0_{\mathbb{C}^{n-2}}$; any of these $k$ sheets is transverse to $Y$ for $U^{\prime}$ small enough. So comes the following:

Proposition 3.9. Let $(X, Y, N)$ satisfying $\left(H_{0}^{r}\right)-\left(H_{3}^{r}\right)$ and let $\mathcal{G}$ be the pseudo-group of local diffeomorphisms that keep invariant the distribution $\mathbb{R} Y$ and any point of $N$ close enough to $x_{0}=0_{\mathbb{R}^{n}} \in N$ (so specially $0_{\mathbb{R}^{n}}$ is invariant). For the equation $\varphi\left(\tilde{\tilde{x}}, x^{n}\right)=0$ of $S_{N} \subset N$ near $x_{0} \in S_{N}$ (where $N$ is identified to $x^{1}=0$ and where $\left.\tilde{\tilde{x}}=\left(x^{2}, \ldots, x^{n-1}\right)^{t}\right), x^{n}$ is of finite order $k \in \mathbb{N}^{*}$ at $0_{\mathbb{R}^{n-1}}$, this order $k$ being invariant under the action of $\mathcal{G}$. And there exists a neighborhood $U^{\prime}$ of $0_{\mathbb{C}^{n-1}}$ such that, if $\tilde{\pi}$ denotes the projection from $\mathbb{C}^{n-2} \times \mathbb{C}$ onto $\mathbb{C}^{n-2}$ defined by $\tilde{\pi}(\tilde{\tilde{Z}}, W)=\tilde{\tilde{Z}}$, the restriction of $\tilde{\pi}$ to $\left\{(\tilde{\tilde{Z}}, W) \in U^{\prime} ; \tilde{\tilde{Z}} \neq 0_{\mathbb{C}^{n-2}}\right.$ and $\left.\varphi(\tilde{\tilde{Z}}, W)=0\right\}$ is a k-sheeted covering map.

If moreover $(X, Y, N)$ satisfies at $x_{0}$ the so-called strong Legendre-Clebsch condition $\frac{\partial}{\partial u} \frac{d^{2}}{d t^{2}} \frac{\partial H}{\partial u}>0$ (where $H$ denotes the Hamiltonian) i.e. from the transversality condition (cf. section 1.3.1) and from computations (see the proof of Lemma 3.4) $\left\langle\vec{n}, a d^{2} Y(X)\left(x_{0}\right)\right\rangle<0$, it comes $k=1$. Then $\varphi(\tilde{\tilde{Z}}, W)=0$ is an analytic submanifold of $\mathbb{C}^{n-1}$, and $S_{N} \subset N$ can be locally (near $x_{0}=0_{\mathbb{R}^{n}} \in S_{N}$ ) identified to $x^{2}=0$ as shown by Figure 11 for $n=3$

In the general case, for any $\tilde{\tilde{x}}_{0} \in \mathbb{R}^{n-2} \backslash\left\{0_{\mathbb{R}^{n-2}}\right\}$ close enough to $0_{\mathbb{R}^{n-2}}$ and for $x^{n}$ in a compact subset $C$ of $\mathbb{R}$, there exists $U^{\prime \prime}$ neighborhood of $\tilde{\tilde{x}}_{0}$ in $\mathbb{R}^{n-2}$ above which $S_{N}$ is the union of $k^{\prime}$ regular submanifolds of $N$ transverse to $Y$, with $k^{\prime} \leq k$ (see Figure 12 for $n=3$ and $k^{\prime}=2$ )

Corollary 3.10. Assume that, for the considered flat problem $\mathcal{P}_{\text {aff }}$ with target $N,(X, Y, N)$ satisfies $\left(H_{0}^{r}\right)-\left(H_{3}^{r}\right)$. Let $\pi$ be the projection defined from $\mathbb{R}^{n-1} \times \mathbb{R}$ onto $\mathbb{R}^{n-1}$ by $\pi\left(\tilde{x}, x^{n}\right)=\tilde{x}$. Using $\pi$, one can generically relate biunivoquely the singular extremals for the original problem $\mathcal{P}_{\text {aff }}$ (with target $N$ ) and for an associated reduced problem (with target $\pi(N)$ ). 


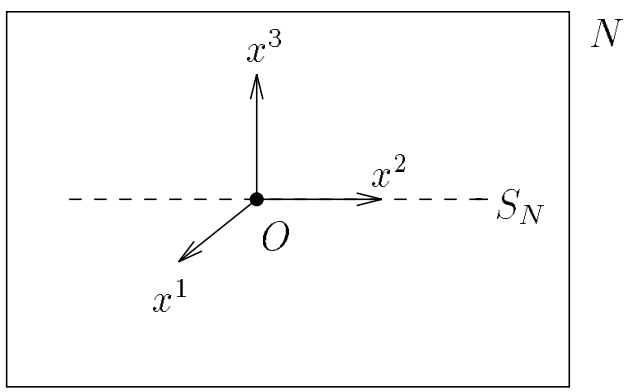

Figure 11
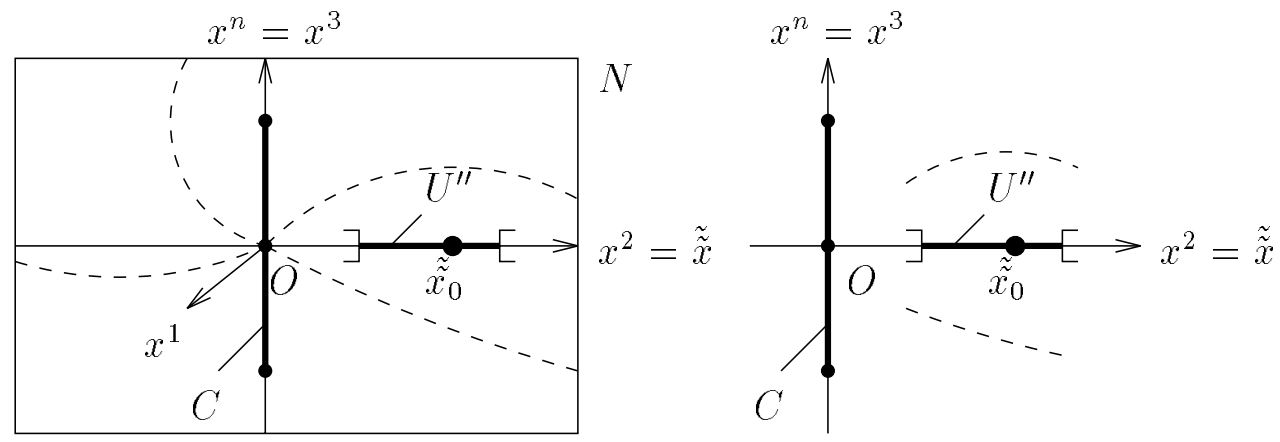

$-\cdots-\cdots S_{N}$

Figure 12

Proof. Since $(X, Y, N)$ satisfies $\left(H_{0}^{r}\right)-\left(H_{3}^{r}\right)$, the restriction of $\dot{x}=X(x)+$ $u Y(x)$ to a neighborhood $U$ of $x_{0}=0_{\mathbb{R}^{n}}$ with local adapted coordinates $\left(\tilde{x}=\left(x^{1}, \ldots, x^{n-1}\right)^{t}, x^{n}\right)$ is:

$$
\dot{\tilde{x}}=\tilde{X}\left(\tilde{x}, x^{n}\right) \quad, \quad \dot{x}^{n}=X^{n}\left(\tilde{x}, x^{n}\right)+u
$$

and as in Lemma 3.7 , one can set $X^{n}=0$ since the concept of singular extremal is feedback invariant.

At final time 0 , singular trajectories for $\mathcal{P}_{a f f}$ arrive on

$$
S_{N}=N \cap(\langle\vec{n},[X, Y](x)\rangle=0)
$$

Indeed, any singular extremal $(x, p, u)$ for $\mathcal{P}_{a f f}$ satisfies: $\langle p, Y(x)\rangle=$ $\langle p,[X, Y](x)\rangle=0$ (cf. section 2.1.4) and the transversality conditions: $x(0) \in N$ and $p(0), \vec{n}$ are colinear (cf. section 1.3.1). Since in $U$ open neighborhood of $0_{\mathbb{R}^{n}}, N$ is $\left(x^{1}=0\right), \vec{n}$ normal vector to $N$ is colinear to $(1,0, \ldots, 0)^{t} \in \mathbb{R}^{n}$; and so the transversality conditions are:

$$
x^{1}(0)=0 \text { and } p_{2}(0)=\cdots=p_{n}(0)=0
$$

Let us associate to $\mathcal{P}_{\text {aff }}$ the time minimal reduced problem $\mathcal{P}_{\text {red }}$ with system $\dot{\tilde{x}}=\tilde{X}\left(\tilde{x}, x^{n}\right)$, control $x^{n}$ and target $\pi(N)$. Computing as in Lemma 3.4, since any singular extremal $(x, p, u)$ for $\mathcal{P}_{a f f}$ satisfies $(3.7)$ and (3.13), we find that $\left(\tilde{x}, \tilde{p}=\left(p_{1}, \ldots, p_{n-1}\right)^{t}, x^{n}\right)$ satisfies (3.8) and that $p_{n} \equiv 0$; so the Hamiltonians $H(x, p, u)=\langle p, X(x)+u Y(x)\rangle$ and $\tilde{H}\left(\tilde{x}, \tilde{p}, x^{n}\right)=\left\langle\tilde{p}, \tilde{X}\left(\tilde{x}, x^{n}\right)\right\rangle$ are equal. Moreover the transversality conditions for $\mathcal{P}_{\text {red }}$ are: $\tilde{x}(0) \in \pi(N)$ 
and $\tilde{p}(0)$ is normal to $\pi(N)$, i.e. (since in $\pi(U)$ open neighborhood of $0_{\mathbb{R}^{n-1}}$, $\pi(N)$ is $\left.\left(x^{1}=0\right)\right) x^{1}(0)=0$ and $p_{2}(0)=\cdots=p_{n-1}(0)=0$, which is equivalent to $(3.13)$ when $p_{n} \equiv 0$.

So $(x, p, u)$ is a singular extremal for $\mathcal{P}_{a f f}$ if and only if $\left(\pi(x), \pi(p), x^{n}\right)$ is a singular extremal for $\mathcal{P}_{\text {red }}$

It appears that $\mathcal{P}_{\text {af } f}$ is a desingularization of $\mathcal{P}_{\text {red }}$ (see Figure 13 for $n=3$ and $k^{\prime}=2$ ) and that, when $k^{\prime}>1$, local maxima of the Hamiltonian $\tilde{H}$ for $\mathcal{P}_{\text {red }}$ may compete at some points of $\pi\left(S_{N}\right)$.

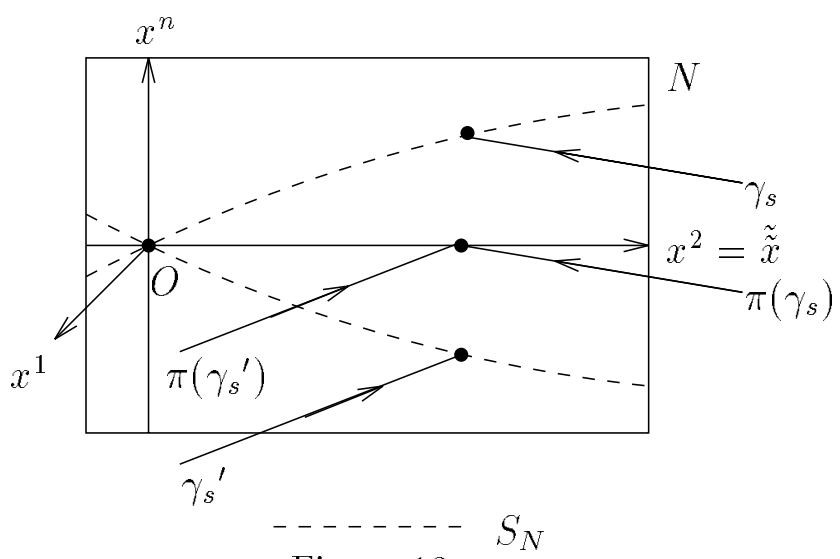

Figure 13

\subsubsection{Applications.}

- Let us consider the flat problem $\dot{\mathcal{P}}_{2}^{\prime}$ in dimension 3 and the associated reduced problem $\mathcal{P}_{2}^{\prime}\left(\mathcal{P}_{2}^{\prime}\right.$ and $\dot{\mathcal{P}}_{2}^{\prime}$ are defined in section 3.1.3). Corollary 3.10 relates biunivoquely the singular extremals for $\dot{\mathcal{P}}_{2}^{\prime}$ and $\mathcal{P}_{2}^{\prime}$.

We shall prove subsequently (cf. Lemma 6.1 in section 6.2.1 and Figure 17) that for $\dot{\mathcal{P}}_{2}^{\prime}$, on the target $N:\left(c_{2}=d_{2}\right)$, there exists a single smooth singular curve transverse to $Y$ : such a flat optimal control problem is said to be simple.

Indeed, keeping the original coordinates $\left(c_{1}, c_{2}\right)$, it comes from sections 1.1 and 3.1 that $\dot{\mathcal{P}}_{2}^{\prime}$ is the time minimal problem associated to the system:

$$
\left\{\begin{array}{l}
\dot{c}_{1}=-v c_{1}^{n_{1}} \\
\dot{c}_{2}=v c_{1}^{n_{1}}-\beta v^{\alpha} c_{2}^{n_{2}} \\
\dot{T}=u \text { which is the control for } \dot{\mathcal{P}}_{2}^{\prime}, \text { while } \mathrm{T} \text { is the control for } \mathcal{P}_{2}^{\prime}
\end{array}\right.
$$

with (cf. equations (1.2) and (1.3))

$$
v=A_{1} \exp \left(-E_{1} / R T\right), \quad \alpha=E_{2} / E_{1}, \quad \beta=A_{2} / A_{1}^{\alpha}
$$

Then assumptions $\left(H_{0}^{r}\right)-\left(H_{3}^{r}\right)$ are satisfied when:

choosing a point $x_{0}=\left(c_{10}, d_{2}, T_{0}\right)$ of

$$
S_{N}=\left\{x=\left(c_{1}, c_{2}=d_{2}, T\right) \in N ; \frac{\partial}{\partial T}\left(v c_{1}^{n_{1}}-\beta v^{\alpha} c_{2}^{n_{2}}\right)=0\right\}
$$

with $\frac{\partial}{\partial T}(v)=E_{1} v / R T^{2}$ and so

$$
S_{N}=\left\{x=\left(c_{1}, c_{2}=d_{2}, T\right) \in N ;\left(c_{1}^{n_{1}}-\alpha \beta v^{\alpha-1} c_{2}^{n_{2}}\right)=0\right\},
$$


and then considering coordinates $\left(x^{1}, x^{2}, x^{3}\right)=\left(c_{2}-d_{2}, c_{1}-c_{10}, T-T_{0}\right)$. Finally $v$ (and so $x^{3}$ ) is of order 1 at $0_{\mathbb{R}^{2}}$ for the equation of $S_{N}$ :

$$
\varphi\left(x^{2}, x^{3}\right)=\left(x^{2}+c_{10}\right)^{n_{1}}-\alpha \beta v^{\alpha-1} d_{2}^{n_{2}}=0
$$

Thus we just proved that the problem $\dot{\mathcal{P}}_{2}^{\prime}$ is simple.

- Let us then consider the time minimal problem for a batch reactor in which three species $X_{1}, X_{2}, X_{3}$ are reacting according to the scheme

$$
X_{1} \leftrightarrows X_{2} \rightarrow X_{3}
$$

each reaction being of the first order, while the final constraint is to obtain a given concentration $d_{2}$ of $X_{2}$.

Similarly as in section 1.1, chemical laws yield the following system:

$$
\left\{\begin{array}{l}
\dot{c}_{1}=-k_{1} c_{1}+k^{\prime}{ }_{1} c_{2} \\
\dot{c}_{2}=k_{1} c_{1}-k^{\prime}{ }_{1} c_{2}-k_{2} c_{2} \\
\dot{c}_{3}=k_{2} c_{2}
\end{array}\right.
$$

with, according to Arrhenius law:

$$
k_{i}=A_{i} \exp \left(-E_{i} / R T\right), i=1,2 \text { and } k^{\prime}{ }_{1}=A^{\prime}{ }_{1} \exp \left(-E^{\prime}{ }_{1} / R T\right)
$$

So, when introducing

$$
v=k_{1}, \alpha=E_{2} / E_{1}, \beta=A_{2} / A_{1}^{\alpha} \alpha^{\prime}=E_{1}^{\prime} / E_{1}, \beta^{\prime}=A_{1}^{\prime} / A_{1}^{\alpha^{\prime}}
$$

and when considering the associated projected problem (cf. section 3.1) with control $T$, we get the following dynamical system

$$
\left\{\begin{array}{l}
\dot{c}_{1}=-v c_{1}+\beta^{\prime} v^{\alpha^{\prime}} c_{2} \\
\dot{c}_{2}=v c_{1}-\beta^{\prime} v^{\alpha^{\prime}} c_{2}-\beta v^{\alpha} c_{2}
\end{array}\right.
$$

whereas the choice of $\dot{T}$ as control 1 gives the supplementary state-variable $T$ satisfying the equation $\dot{T}=u$.

Then assumptions $\left(H_{0}^{r}\right)-\left(H_{3}^{r}\right)$ are satisfied when:

choosing a point $x_{0}=\left(c_{10}, d_{2}, T_{0}\right)$ of

$$
S_{N}=\left\{x=\left(c_{1}, c_{2}=d_{2}, T\right) \in N ; \frac{\partial}{\partial T}\left(v c_{1}-\beta^{\prime} v^{\alpha^{\prime}} c_{2}-\beta v^{\alpha} c_{2}\right)=0\right\}
$$

with $\frac{\partial}{\partial T}(v)=E_{1} v / R T^{2}$ and so

$$
S_{N}=\left\{x=\left(c_{1}, c_{2}=d_{2}, T\right) \in N ; c_{1}-\alpha^{\prime} \beta^{\prime} v^{\alpha^{\prime}-1} c_{2}-\alpha \beta v^{\alpha-1} c_{2}=0\right\},
$$

and then considering coordinates $\left(x^{1}, x^{2}, x^{3}\right)=\left(c_{2}-d_{2}, c_{1}-c_{10}, T-T_{0}\right)$. Finally the equation of $S_{N}$ becomes:

$$
\varphi\left(x^{2}, x^{3}\right)=x^{2}+c_{10}-\left(\alpha^{\prime} \beta^{\prime} v^{\alpha^{\prime}-1}+\alpha \beta v^{\alpha-1}\right) d_{2}=0
$$

So $v$ (and so $x^{3}$ ) is of order 2 at $0_{\mathbb{R}^{2}}$ for this equation of $S_{N}$ when we choose $\alpha=2$ and $\alpha^{\prime}=3$; thus we just proved that in that case the considered problem is not simple. 


\section{TIME MINIMAL SYNTHESIS FOR $\mathcal{P}_{2}$ NEAR THE TARGET}

\subsection{Generalities}

4.1.1. Definitions and notations. According to section 3.1.3 we can consider instead of $\mathcal{P}_{2}$ the equivalent time minimal projected problem $\mathcal{P}_{2}^{\prime}$ with system (3.4), control $v \in\left[v_{m}, v_{M}\right]$ and terminal manifold $N:\left(c_{2}=d_{2}\right)$ to be reached from an initial value $\left(c_{2}\right)_{0} \in\left[0, d_{2}[\right.$. So the state-vector is $\tilde{x}:=\left(x_{1}, c_{2}\right)^{t}, x_{1}$ being related to the original variable $c_{1}$ by (3.2). Note that if $n_{1}>1$ it comes $x_{1}<0$ since (cf. (1.6) in section 1.1.3) $c_{1}>0$.

Let us denote by $\hat{v}$ the singular control, and by $\gamma_{m}$ (resp. $\gamma_{M}, \hat{\gamma}$ ) any arc satisfying (3.4) with control $v_{m}$ (resp. $\left.v_{M}, \hat{v}\right)$.

4.1.2. Adjoint-STATe. Along any $B C$-extremal $(\tilde{x}, \tilde{p}, v)$, the adjoint-vector $\tilde{p}=\left(p_{1}, p_{2}\right)^{t}$ associated to $\tilde{x}=\left(x_{1}, c_{2}\right)^{t}$ satisfies (when taking (3.2) into account):

$$
\left\{\begin{array}{l}
\dot{p}_{1}=-n_{1} v c_{1}^{2 n_{1}-1} p_{2} \\
\dot{p}_{2}=n_{2} \beta v^{\alpha} c_{2}^{n_{2}-1} p_{2}
\end{array}\right.
$$

the corresponding Hamiltonian being:

$$
\tilde{H}(\tilde{x}, \tilde{p}, v)=-v p_{1}+\left(v c_{1}^{n_{1}}-\beta v^{\alpha} c_{2}^{n_{2}}\right) p_{2}
$$

At final time 0 we can take

$$
\tilde{p}(0)=(0,1)
$$

and at any negative time $t, p_{1}(t)>0$ and $p_{2}(t)>0$.

Proof. Since the target $N:\left(c_{2}=d_{2}\right)$ has to be reached from the domain $\left(c_{2} \in\left[0, d_{2}[)\right.\right.$, the PMP (maximization of the Hamiltonian and transversality conditions) implies in the non-exceptional case that $\tilde{p}(0)$ is a normal vector to $N$ pointing outwards $\left(c_{2}<d_{2}\right)$; so we can take $\tilde{p}(0)=(0,1)$.

Note that in the exceptional case (i.e. [cf. section 1.3.2] when the Hamiltonian is zero, or equivalently when the trajectory is tangent to $N$ at final time 0$)$ PMP allows $\tilde{p}(0)=(0,-1)$ as well as $\tilde{p}(0)=(0,1)$; the value $(0,-1)$ is discarded for optimal trajectories (this result is proved in the same way as the orientation principle [2]).

From (4.1), at any time $t, p_{2}(t)=\left[\exp \int_{0}^{t} n_{2} \beta v^{\alpha}(u) c_{2}^{n_{2}-1}(u) d u\right] p_{2}(0)$; and from $(4.3) p_{2}(0)=1>0$, so $p_{2}(t)>0$ : this implies that at any negative time $t \dot{p}_{1}(t)<0$ (from (4.1), with $c_{1}(t)>0$ from (1.6) in section 1.1.3, $\left.0<v_{m} \leq v(t)\right)$; so, since from $(4.3) p_{1}(0)=0$, it comes $p_{1}(t)>0$.

4.1.3. Accessibility. Let $A$ be the set of points of the target $N:\left(c_{2}=d_{2}\right)$ that are accessible from $\left(c_{2}>d_{2}\right)$. When $\alpha<1$ (resp. $\left.\alpha>1\right), A$ is included in $A^{\prime}=\left\{\left(c_{1}, d_{2}\right) \in N ; c_{1} \geq c_{1 M}\right.$ (resp. $\left.\left.c_{1 m}\right)\right\}$ where $c_{1 M}=\left(\beta d_{2}^{n_{2}} v_{M}^{\alpha-1}\right)^{\frac{1}{n 1}}$ (resp. $c_{1 m}=\left(\beta d_{2}^{n_{2}} v_{m}^{\alpha-1}\right)^{\frac{1}{n_{1}}}$ ) (see Figures 4 and 5 in section 1.4.2).

Proof. For any $v_{0} \in\left[v_{m}, v_{M}\right]$ (so $v_{0}>0$ ), any point $a=\left(x_{1}, d_{2}\right)$ of $A$ reached with final control $v_{0}$ has to satisfy $\tilde{H}\left(a, \tilde{p}(0), v_{0}\right) \geq 0$, i.e. (from (4.2) and (4.3) in section 4.1.2) $v_{0} c_{1}^{n_{1}}-\beta v_{0}^{\alpha} d_{2}^{n_{2}} \geq 0$, i.e. $c_{1} \geq\left(\beta d_{2}^{n_{2}} v_{0}^{\alpha-1}\right)^{\frac{1}{n_{1}}}$, and so ESAIM: CoCV, DeCEMBER 1998, Vol. 3, 407-467 
comes the result since $\alpha-1<0$ and $0<v_{0} \leq v_{M}$ (resp. since $\alpha-1>0$ and $\left.0<v_{m} \leq v_{0}\right)$.

4.1.4. Singular $B C$-eXtremals. There is no singular BC-extremal when $\alpha<1$; indeed the (necessary) Legendre condition is satisfied if and only if $\alpha>1$.

Proof. From section 1.3.4 and (4.2) any singular BC-extremal has to satisfy the Legendre condition $\left.\frac{\partial^{2} \tilde{H}}{\partial v^{2}}\right|_{\tilde{x}, \tilde{p}, \nu)} \leq 0$, i.e. $-\alpha(\alpha-1) \beta v^{\alpha-2} c_{2}^{n_{2}} p_{2} \leq 0$, which is true if and only if $\alpha>1$ since at any non-positive time $t p_{2}(t)>0$ (cf. section 4.1.2), $v(t) \geq v_{m}>0$, and $c_{2}(t) \geq 0$ from (1.6) in section 1.1.3 with $c_{2}(t)>0$ if $t$ is not the initial time.

\subsection{LOCAL SYNTHESIS FOR $\alpha<1$}

THEOREM 4.1. Locally (near the target $N$ ), as shown by Figure 4 in section 1.4.2, the optimal trajectories are the $\gamma_{M}$ arcs arriving on $A^{\prime}=\left\{\left(c_{1}, d_{2}\right) \in\right.$ $\left.N ; c_{1} \geq c_{1 M}\right\}$ defined in section 4.1.3. Note that the point $E_{M}$ defined in section 1.4.2 as the point of the target such that $\dot{c}_{2}=0$ when $v=v_{M}$, is $E_{M}=\left(c_{1 M}, d_{2}\right)$ arrival point of an optimal exceptional trajectory $\gamma_{M}$.

Proof. When $\alpha<1$, we know from section 4.1.4 that any $B C$-extremal $(\tilde{x}, \tilde{p}, v)$ is regular.

Let us assume that $c_{2}>0$, which is not restrictive in this local study near $N:\left(c_{2}=d_{2}>0\right)$.

Moreover, for any $B C$-extremal $(\tilde{x}, \tilde{p}, v)$, it comes from (4.2) in section 4.1.2:

$$
\left.\frac{\partial \tilde{H}}{\partial v}\right|_{(\tilde{x}, \tilde{p}, v)}=-p_{1}+\left(c_{1}^{n_{1}}-\alpha \beta v^{\alpha-1} c_{2}^{n_{2}}\right) p_{2}
$$

with

$$
-p_{1}+c_{1}^{n_{1}} p_{2}>0
$$

since $\tilde{H}(\tilde{x}, \tilde{p}, v)=v\left(-p_{1}+c_{1}^{n_{1}} p_{2}\right)-\beta v^{\alpha} c_{2}^{n_{2}} p_{2}<v\left(-p_{1}+c_{1}^{n_{1}} p_{2}\right)$ [indeed $v \geq v_{m}>0$, we assumed that $c_{2}>0$, and $p_{2}$ is positive valued from 4.1.2] and [from PMP] $\tilde{H}(\tilde{x}, \tilde{p}, v) \geq 0$ with $v \geq v_{m}>0$.

Let us then introduce

$$
\hat{v}=\left[\frac{1}{\alpha \beta c_{2}^{n_{2}}}\left(c_{1}^{n_{1}}-\frac{p_{1}}{p_{2}}\right)\right]^{\frac{1}{\alpha-1}}
$$

which is the solution to the equation in $v: \frac{\partial \tilde{H}}{\partial v}(\tilde{x}, \tilde{p}, v)=0$.

Let us consider a fixed $(\tilde{x}, \tilde{p})$ satisfying $(4.5)$ and such that $c_{1}>0, c_{2}>0$, $p_{1} \geq 0$ and $p_{2}>0$; let us look then for the variations of $v \mapsto \tilde{H}(\tilde{x}, \tilde{p}, v)$ for $v>0$ :

from (4.2) in section 4.1 .2 it comes

$$
\lim _{v \rightarrow 0^{+}} \tilde{H}(\tilde{x}, \tilde{p}, v)=0
$$


and, since moreover $\alpha<1$, we get:

$$
\begin{aligned}
& \frac{\partial^{2} \tilde{H}}{\partial v^{2}}(\tilde{x}, \tilde{p}, v)=-\alpha(\alpha-1) \beta v^{\alpha-2} c_{2}^{n_{2}} p_{2}>0 \\
& \lim _{v \rightarrow+\infty} \tilde{H}(\tilde{x}, \tilde{p}, v)=\lim _{v \rightarrow+\infty} v\left[-p_{1}+c_{1}^{n_{1}} p_{2}-\beta v^{\alpha-1} c_{2}^{n_{2}} p_{2}\right]=+\infty
\end{aligned}
$$

So comes the following table 1 which gives the variations of $v \mapsto \tilde{H}(\tilde{x}, \tilde{p}, v)$ for $v>0$.

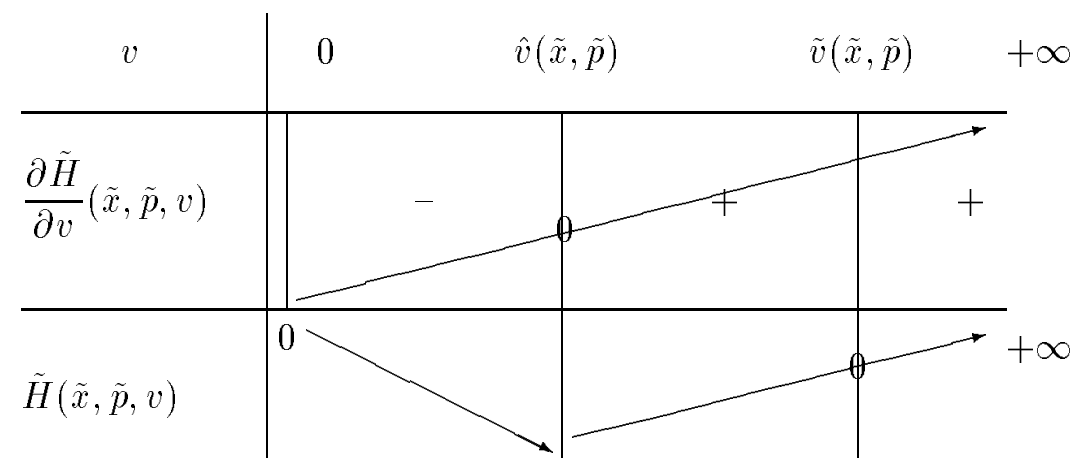

Table 1

Computing as in the proof of result 4.1.3, we find that at any point $a=\left(c_{1}, d_{2}\right)$ of $A^{\prime}$ with $\tilde{p}(0)=(0,1)$ according to (4.3) in section 4.1.2, $\tilde{H}\left(a, \tilde{p}(0), v_{M}\right) \geq 0$ and that $\tilde{H}\left(a, \tilde{p}(0), v_{M}\right)=0$ if and only if $c_{1}=c_{1 M}$. Then:

- for any point $a=\left(c_{1}, d_{2}\right)$ of $A^{\prime}$ such that $c_{1}>c_{1 M}$ we know from Table 1 that $v_{M}>\tilde{v}(a, \tilde{p}(0))$ and that $v_{M}$ maximizes $v \mapsto \tilde{H}(a, \tilde{p}(0), v)$ for $v \in\left[v_{m}, v_{M}\right]$. So locally the $\gamma_{M}$ trajectory arriving at $a$ is $B C$ extremal.

- since $\tilde{p}(0)=(0,1), \tilde{H}\left(a, \tilde{p}(0), v_{M}\right)$ is equal to $\dot{c}_{2}$ at point a with $v=v_{M}$; so $\left(c_{1 M}, d_{2}\right)$ is the point of the target such that $\dot{c}_{2}=0$ with $v=v_{M}$, i.e. $\left(c_{1 M}, d_{2}\right)=E_{M}$ introduced in section 1.4 .2 , and the arc $\gamma_{M}$ arriving at $E_{M}$ is tangent to $N$.

And since $\tilde{H}\left(E_{M}, \tilde{p}(0), v_{M}\right)=0$, we know from Table 1 that $\tilde{v}\left(E_{M}, \tilde{p}(0)\right)=$ $v_{M}$ and that $v_{M}$ maximizes $v \mapsto \tilde{H}\left(E_{M}, \tilde{p}(0), v\right)$ for $v \in\left[v_{m}, v_{M}\right]$.

Moreover, in that case, $\dot{c}_{2}(0)=0$ with $v=v_{M}$ and $\tilde{x}(0)=E_{M}$, i.e. (cf. section 4.1.3) $c_{1}(0)=c_{1 M}$ and $c_{2}(0)=d_{2}$. So, since from (1.4) in section 1.1.2

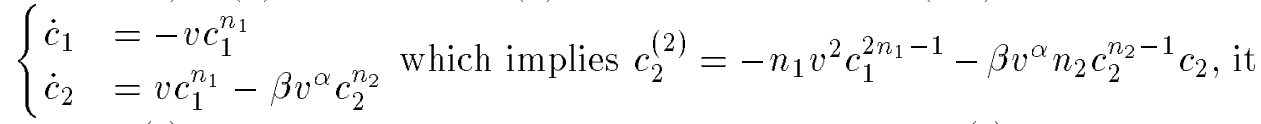
comes $c_{2}^{(2)}(0)=-n_{1} v_{M}^{2} c_{1 M}^{2 n_{1}-1}<0$. Since $\dot{c}_{2}(0)=0$ and $c_{2}^{(2)}(0)<0$, we get from Taylor's expansion of $c_{2}$ at time 0 that for any time $t$ close enough to $0 c_{2}(t)<c_{2}(0)=d_{2}$.

So the $\gamma_{M}$ trajectory arriving at $E_{M}$ is locally $B C$-extremal.

Recapitulating, we get the result. 


\subsection{LOCAL SYNTHESIS FOR $\alpha>1$}

4.3.1. Stratification of the target $N$. Locally (near the target $N$ ) as shown by Figure 5 in section 1.4.2 any point of $A^{\prime}=\left\{\left(c_{1}, d_{2}\right) \in N ; c_{1} \geq\right.$ $\left.c_{1 m}\right\}$ defined in section 4.1 .3 is the arrival point of a $B C$-extremal trajectory. The point $E_{m}$, defined in section 1.4.2 as the point of $N$ such that $\dot{c}_{2}=0$ when $v=v_{m}$, is $E_{m}=\left(c_{1 m}, d_{2}\right)$ arrival point of an exceptional BC-extremal trajectory with final control $v_{m}$.

The point $S_{m}$ (resp. $S_{M}$ ) is, as in section 1.4.D, the point of $N$ where the final singular control is $v_{m}$ (resp. $\left.v_{M}\right)$.

The final control of the BC-extremal trajectories arriving on $N$ is then shown by Figure 14

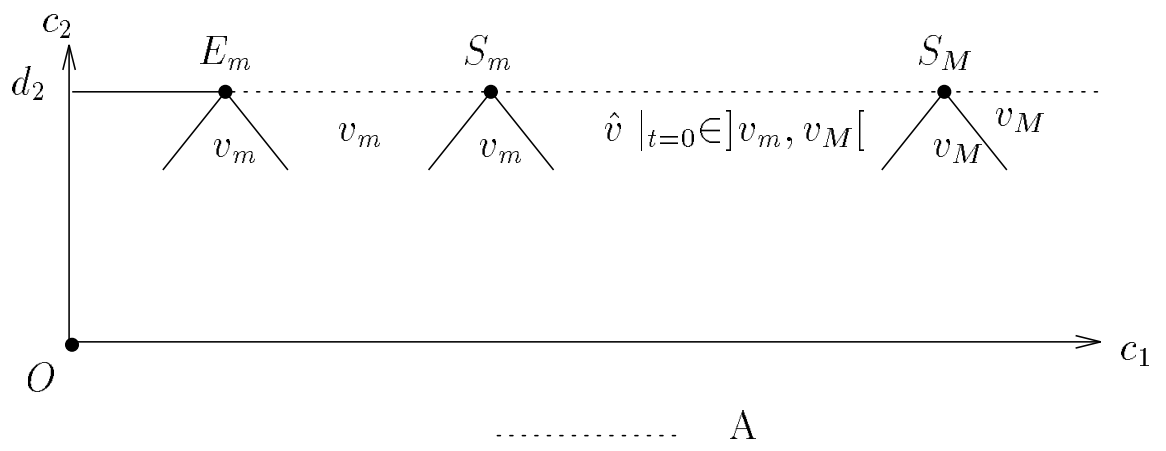

Figure 14

Proof. As in the proof of Theorem 4.1:

We assume that $c_{2}>0$, that any $B C$-extremal $(\tilde{x}, \tilde{p}, v)$ satisfies $(4.4)$ and (4.5), and we consider $\hat{v}$ defined by (4.6) which is the solution to the equation in $v: \frac{\partial \tilde{H}}{\partial v}(\tilde{x}, \tilde{p}, v)=0$.

Let us consider a fixed $(\tilde{x}, \tilde{p})$ satisfying (4.5) and such that $c_{1}>0, c_{2}>0$, $p_{1} \geq 0$ and $p_{2}>0$; let us look then for the variations of $v \mapsto \tilde{H}(\tilde{x}, \tilde{p}, v)$ for $v>0$ :

from (4.2) in section 4.1.2 it comes $\lim _{v \rightarrow 0^{+}} \tilde{H}(\tilde{x}, \tilde{p}, v)=0$; and since moreover $\alpha>1$, we get:

$$
\begin{gathered}
\frac{\partial^{2} \tilde{H}}{\partial v^{2}}(\tilde{x}, \tilde{p}, v)=-\alpha(\alpha-1) \beta v^{\alpha-2} c_{2}^{n_{2}} p_{2}<0 \\
\lim _{v \rightarrow+\infty} \tilde{H}(\tilde{x}, \tilde{p}, v)=\lim _{v \rightarrow+\infty} v^{\alpha}\left[-\beta c_{2}^{n_{2}} p_{2}+v^{1-\alpha}\left(-p_{1}+c_{1}^{n_{1}} p_{2}\right)\right]=-\infty
\end{gathered}
$$

So comes the following table 2 which gives the variations of $v \mapsto \tilde{H}(\tilde{x}, \tilde{p}, v)$ for $v>0$.

Computing as in the proof of result 4.1.3, we find that at any point $a=$ $\left(c_{1}, d_{2}\right)$ of $A^{\prime}$, with $\tilde{p}(0)=(0,1)$ according to $(4.3)$ in section 4.1.2, $\tilde{H}\left(a, \tilde{p}(0), v_{m}\right) \geq 0$ and that $\tilde{H}\left(a, \tilde{p}(0), v_{m}\right)=0$ if and only if $c_{1}=c_{1 m}$.

Moreover (4.6) implies that $\left.\hat{v}\right|_{t=0}$, which is $\hat{v}(a, \tilde{p}(0))$, is equal to $\left(\frac{c_{1}^{n_{1}}}{\alpha \beta d_{2}^{n_{2}}}\right)^{\frac{1}{\alpha-1}}$ and so is an increasing function of $c_{1}$. 


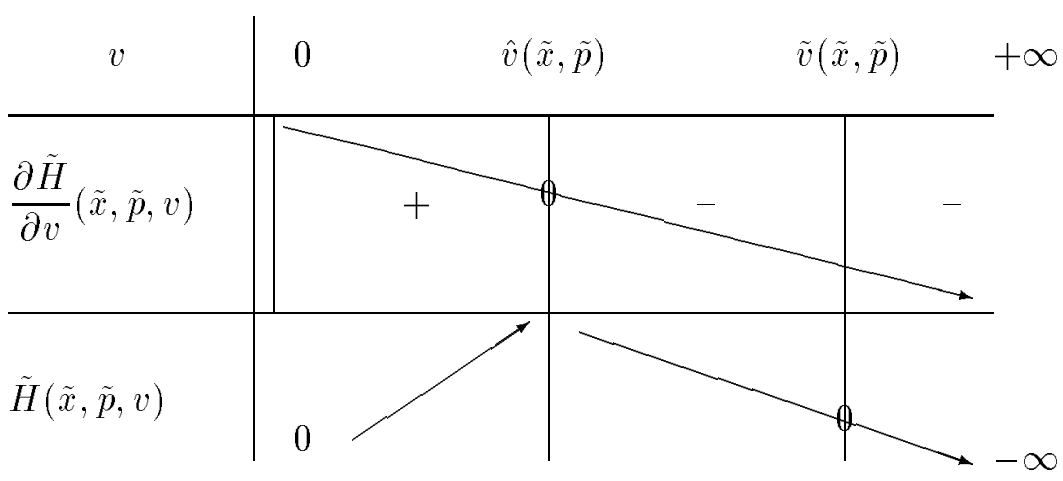

Table 2

Then for any point $a=\left(c_{1}, d_{2}\right)$ of $A^{\prime}$ such that $c_{1}>c_{1 m}$ we know from Table 2 that $v_{m}<\tilde{v}(a, \tilde{p}(0))$ and that $v \mapsto \tilde{H}(a, \tilde{p}(0), v)$ for $v \in\left[v_{m}, v_{M}\right]$ is maximized by:

- $v_{m}$ if $\left.\hat{v}\right|_{t=0} \leq v_{m}$, i.e. if $\left.\left.a \in\right] E_{m}, S_{m}\right]$

- $\left.\hat{v}\right|_{t=0}$ if $\left.\left.\hat{v}\right|_{t=0} \in\right] v_{m}, v_{M}[$, i.e. if $a \in] S_{m}, S_{M}[$

- $v_{M}$ if $\left.\hat{v}\right|_{t=0} \geq v_{M}$, i.e. if $a$ is on the right of $S_{M}$.

The proof of the results claimed about $E_{m}$ is similar to the proof given in section 4.2 about $E_{M}$.

\subsubsection{Synthesis.}

THEOREM 4.2. Each optimal trajectory is of the form $\gamma_{M} \hat{\gamma} \gamma_{m}$, where each arc of the sequence may be empty, and each open-loop control is $\mathcal{C}^{0}$.

Numerical simulations (cf. section 8) show that the optimal synthesis is given by Figure 5 in section 1.4.2.

Proof. As in the proofs of sections 4.2 and 4.3.1 we assume that $c_{2}>0$. Along any $B C$-extremal $(\tilde{x}, \tilde{p}, v)$, let us study the variations of the singular control $\hat{v}$ (cf. (4.6) in section 4.2) which are the same as the variations of $\hat{w}=\alpha \beta \hat{v}^{\alpha-1}$ since $\alpha>1$ and $\beta>0$.

From (4.6) it comes $\hat{w}=\frac{1}{c_{2}^{n_{2}}}\left(c_{1}^{n_{1}}-\frac{p_{1}}{p_{2}}\right)$ and so computations taking into account (1.4) in section 1.1.2 and (4.1), (4.2) in section 4.1.2 yield:

$$
\frac{d \hat{w}}{d t}=-\frac{n_{2} c_{1}^{n_{1}} \tilde{H}(\tilde{x}, \tilde{p}, v)}{c_{2}^{n_{2}+1} p_{2}}
$$

Moreover we know that: $c_{1}>0$ (cf. (1.6) in section 1.1.3), $p_{2}>0$ (cf. section 4.1.2) and that $t \mapsto \tilde{H}(\tilde{x}(t), \tilde{p}(t), v(t))$ is a non-negative constant (cf. $\mathrm{PMP})$. And we assumed that $c_{2}>0$.

If $\tilde{H}(\tilde{x}, \tilde{p}, v) \equiv 0$, which (from section 4.3.1) occurs only for the exceptional $B C$-extremal arriving at $E_{m}$ with final control $v_{m}, \hat{w}$ is constant along this $B C$-extremal and so does $\hat{v}$. So, considering Table 2 in section 4.3.1, at any non-positive time $t$ close enough to $0, \hat{v}<v_{m}$ since $\left.\hat{v}\right|_{t=0}<v_{m}=$ $\tilde{v}\left(E_{m}, \tilde{p}(0)\right)$; and so $v_{m}=\tilde{v}(\tilde{x}(t), \tilde{p}(t))$ maximizes $v \mapsto \tilde{H}(\tilde{x}(t), \tilde{p}(t), v)$. Thus $E_{m}$ is the arrival point of an optimal exceptional trajectory $\gamma_{m}$. 
If $\tilde{H}(\tilde{x}, \tilde{p}, v)>0$, which (from section 4.3.1) occurs for $B C$-extremals arriving on $N$ strictly on the right of $E_{m}, \hat{w}$ is a decreasing function of time along any of these $B C$-extremals and so does $\hat{v}$.

We already noticed in section 4.1 .4 that $c_{2} \geq 0$ with $c_{2}(t)>0$ if $t$ is not the initial time $t_{0}$ (from (1.6) in section 1.1.3); and we assumed in sections 4.2 and 4.3 that $c_{2}>0$. If $c_{2}\left(t_{0}\right)=0$, it comes from section 4.1.2 and (4.6) that $\lim _{t \rightarrow t_{0}^{+}} \hat{v}(\tilde{x}(t), \tilde{p}(t))=+\infty$; otherwise let us prove that $\lim _{t \rightarrow t^{+}} \hat{v}(\tilde{x}(t), \tilde{p}(t))=+\infty$ with $t_{0}=-\infty$, so that we can conclude in any case that $\hat{v}$ relates biunivoquely and decreasingly the considered times $\left.] t_{0}, 0\right]$ onto $\left[\left.\hat{v}\right|_{t=0},+\infty[\right.$. Indeed at any time $t \leq 0$ :

- in the considered case $t \mapsto \tilde{H}(\tilde{x}(t), \tilde{p}(t), v(t))$ is a positive constant (say $\tilde{H})$; and $c_{2}(t)>0$;

- moreover $c_{1}(t)>0$ [from (1.6) in section 1.1.3]; and $p_{1}(t) \geq 0, p_{2}(t)>0$ [from section 4.1.2].

- So (1.4) in section 1.1.2, (4.1) and (4.2) in section 4.1.2 imply that $\dot{c}_{1}(t)<0, \dot{p}_{2}(t)>0,0<\tilde{H} \leq \dot{c}_{2}(t) p_{2}(t)$ and so $\dot{c}_{2}(t)>0$. Thus, since $p_{2}(0)=1$ from (4.3) in section 4.1.2, it comes from (4.7):

$$
\frac{d \hat{w}}{d t} \leq-\frac{n_{2} c_{1}^{n_{1}}(0) \tilde{H}}{c_{2}^{n_{2}+1}(0)} \text { which is a negative constant }
$$

so we get $\lim _{t \rightarrow-\infty} \hat{w}(\tilde{x}(t), \tilde{p}(t))=+\infty$, and so $\lim _{t \rightarrow-\infty} \hat{v}(\tilde{x}(t), \tilde{p}(t))=+\infty$.

Finally we find the following results:

- if $\left.\hat{v}\right|_{t=0} \geq v_{M}$, which (from section 4.3.1) occurs for $B C$-extremals arriving on the right of $S_{M}$, at any time $\left.\left.t \in\right] t_{0}, 0\right]$ close enough to $0, \hat{v} \geq v_{M}$ and so (from Table 2 in section 4.3.1) $v_{M}$ maximizes $v \mapsto \tilde{H}(\tilde{x}(t), \tilde{p}(t), v)$. Thus any point on the right of $S_{M}$ is the arrival point of an optimal trajectory $\gamma_{M}$,

- if $\left.\hat{v}\right|_{t=0} \in\left[v_{m}, v_{M}\right.$, which (from section 4.3.1) occurs for $B C$-extremal arriving on $\left[S_{m}, S_{M}\left[\right.\right.$, there exists a single time $\left.t_{M} \in\right] t_{0}, 0\left[\right.$ such that $\left.\hat{v}\right|_{t=t_{M}}=$ $v_{M}$. And at any time $\left.\left.t \in\right] t_{0}, 0\right]$ close enough to 0 : for $t \leq t_{M}$ (resp. $t>t_{M}$ ), $\hat{v} \geq v_{M}$ (resp. $\hat{v} \in\left[v_{m}, v_{M}\left[\right.\right.$ ) and so (from Table 2 in section 4.3.1) $v_{M}$ (resp. $\hat{v})$ maximizes $v \mapsto \tilde{H}(\tilde{x}(t), \tilde{p}(t), v)$. Thus any point of $\left[S_{m}, S_{M}[\right.$ is the arrival point of an optimal trajectory $\gamma_{M} \hat{\gamma}$,

- if $\left.\hat{v}\right|_{t=0}<v_{m}$, which (from section 4.3.1) occurs in the considered case for $B C$-extremals arriving on $] E_{m}, S_{m}\left[\right.$, there exists a single time $t_{M}$ (resp. $t_{m}$ ) in $] t_{0}, 0\left[\right.$ such that $\left.\hat{v}\right|_{t=t_{M}}=v_{M}$ (resp. $\left.\hat{v}\right|_{t=t_{m}}=v_{m}$ ). And at any time $\left.t \in] t_{0}, 0\right]$, close enough to 0 : for $t \leq t_{M}$ (resp. $\left.t \in\right] t_{M}, t_{M}\left[, t \geq t_{m}\right), \hat{v} \geq v_{M}$ (resp. $\hat{v} \in] v_{m}, v_{M}\left[, \hat{v} \leq v_{m}\right.$ ) and so (from Table 2 in section 4.3.1), $v_{M}$ (resp. $\hat{v} \in] v_{m}, v_{M}\left[, v_{m}\right)$ maximizes $v \mapsto \tilde{H}(\tilde{x}(t), \tilde{p}(t), v)$. Thus any point of ]$E_{m}, S_{m}$ [ is the arrival point of an optimal trajectory $\gamma_{M} \hat{\gamma} \gamma_{m}$. 
5. Time minimal synthesis For $\dot{\mathcal{P}}_{1}$ IN The CASE $n_{1}=n_{2}=1$

\subsection{Generalities}

5.1.1. Definitions and notations. According to section 3.1.3 we can consider instead of $\dot{\mathcal{P}}_{1}$ in the case $n_{1}=n_{2}=1$ the equivalent time minimal projected problem $\dot{\mathcal{P}}_{1}^{\prime}$ with system (1.18) (cf. section 1.4.1), control $u \in\left[u_{-}, u_{+}\right]$with $u_{-}<0<u_{+}$and terminal manifold $N:\left(z=d_{1}\right)$ to be reached from an initial value $z_{0} \in\left[0, d_{1}\right.$ [. So the state-vector is $x=(z, v)^{t}$, with $v \in] 0, A_{1}\left[\right.$ (cf. (1.6) in section 1.1.3). Let us denote by $u_{s}$ the singular control and by $\gamma_{-}$(resp. $\gamma_{+}, \gamma_{s}$ ) any arc satisfying (1.18) with control $u_{-}$ (resp. $u_{+}, u_{s}$ ).

5.1.2. Adjoint-STate. Along any $B C$-extremal $(x, p, u)$, the adjoint vector $p=\left(p_{1}, p_{2}\right)^{t}$ associated to $x=(z, v)^{t}$ satisfies:

$$
\left\{\begin{array}{l}
\dot{p}_{1}=\left(\beta v^{\alpha}-v\right) p_{1} \\
\dot{p}_{2}=\left(\alpha \beta v^{\alpha-1} z-z-1\right) p_{1}-\frac{d h}{d v}(v) u p_{2}
\end{array}\right.
$$

the corresponding Hamiltonian being

$$
H(x, p, u)=\left(v+v z-\beta v^{\alpha} z\right) p_{1}+h(v) u p_{2}
$$

At final time 0 , we can take

$$
p(0)=(1,0)
$$

and at any non positive time $t, p_{1}(t)>0$.

Proof. Equations (5.1), (5.2) come from section 1.3.1 applied to the optimal control problem $\dot{\mathcal{P}}_{1}^{\prime}$ stated in section 5.1.1.

The proof of $(5.3)$ is similar to the proof of $\tilde{p}(0)=(0,1)$ in section 4.1.2. And from (5.1), at any non-positive time $t, p_{1}(t)=\left[\exp \int_{0}^{t}\left(\beta v^{\alpha}(u)-\right.\right.$ $v(u)) d u] p_{1}(0)$ with $p_{1}(0)=1>0$, so $p_{1}(t)>0$.

5.1.3. Accessibility. Let $A$ be the set of points of the target $N:\left(z=d_{1}\right)$ that are accessible from $\left(z<d_{1}\right)$. Let $G$ be the set of points $(z>0, v \in$ ]0, $A_{1}[$ ) such that $\dot{z}=0$ (cf. (1.18) in section 1.4.1) as in section 1.4.1.

When $\alpha<1$ (resp. $\alpha>1$ ), $A$ is included in $A^{\prime}=\left\{\left(d_{1}, v \in\right] 0, A_{1}[) \in\right.$ $N ; v \geq v_{A}$ (resp. $\left.\left.v \leq v_{A}\right)\right\}$ where $v_{A}=\left(\frac{\beta d_{1}}{1+d_{1}}\right)^{\frac{1}{1-\alpha}}$ is the v-coordinate of $G \cap\left(z=d_{1}\right)$. Note that when $\alpha<1$ and $v_{A} \geq A_{1}, A^{\prime}$ is empty and so $A$ is empty.

Proof. For any $u_{0} \in\left[u_{-}, u_{+}\right]$, any point $a=\left(d_{1}, v\right)$ of $A$ reached with final control $u_{0}$ has to satisfy $H\left(a, p(0), u_{0}\right) \geq 0$, i.e. from $(5.2),(5.3)$ in section $5.1 .2 v+v d_{1}-\beta v^{\alpha} d_{1} \geq 0$ i.e. (since $\beta d_{1}>0$ from section 1.1 and since $v>0$ from (1.6) in section 1.1.3) $v^{\alpha-1} \leq \frac{1+d_{1}}{\beta d_{1}}$, and so comes the result according to the sign of $\alpha-1$. Note that $v+v d_{1}-\beta v^{\alpha} d_{1}=\left.\dot{z}\right|_{x=a}$ and so $v=v_{A}$ is equivalent to $\dot{z}=0$ at the arrival point $a$. 
5.1.4. SWITCHIng FUnCtion. The switching function of $\dot{\mathcal{P}}_{1}^{\prime}$ is

$$
\Phi: t \mapsto h(v(t)) p_{2}(t)
$$

Thus (5.3) in section 5.1.2 implies that $\Phi(0)=0$. Indeed we can notice that problem $\dot{\mathcal{P}}_{1}^{\prime}$ is flat.

Then at any time:

$$
\dot{\Phi}=-h(v)\left(1+z-\alpha \beta v^{\alpha-1} z\right) p_{1}
$$

Proof. Computations, applying equations (2.2)-(2.4) of section 2.1.2 to problem $\dot{\mathcal{P}}_{1}^{\prime}$ stated in section 5.1.1 with affine single-input system (1.18).

5.1.5. Singular $B C$-extremals. As in section 1.4.1, let $S$ be the set of points $(z>0, v \in] 0, A_{1}[)$ such that $\frac{d}{d t} \frac{\partial H}{\partial u}=0$.

LEMMA 5.1. Any singular arc $\gamma_{s}$ of a $B C$-extremal trajectory is included in $S$, with $S=\left\{(z>0, v \in] 0, A_{1}[)\right.$ such that $\left.z\left(\alpha \beta v^{\alpha-1}-1\right)=1\right\}$; the singular control is $u_{s}=-\frac{v^{2}}{\alpha z h(v)}<0$ and so $v$ is a decreasing function of time along $\gamma_{s}$.

Proof. From section 1.3.4 any singular $B C$-extremal has to satisfy $\frac{d}{d t} \frac{\partial H}{\partial u}=$ 0 , so $\gamma_{s}$ is included in $S$.

From (5.2) in section 5.1.2, $\frac{d}{d t} \frac{\partial H}{\partial u}=0$ is equivalent to $\frac{d}{d t}\left(h(v) p_{2}\right)=0$ which is equivalent from (1.18) in section 1.4 .1 and (5.1) in section 5.1.2 to $\left(\alpha \beta v^{\alpha-1} z-z-1\right) h(v) p_{1}=0$. We thus find the expected equation of $S$ since $h(v)$ is always positive (from (1.7) in section 1.1.3) and since $p_{1}$ is never zero (from section 5.1.2).

Applying (2.5) (cf. section 2.1.4) to system (1.18), we get the value of the singular control $u_{s}$ which is negative since $\alpha>0$ (from section 1.1) and $v^{2} z h(v)>0$ (from $(1.6),(1.7)$ in section 1.1.3). Finally, since $\dot{v}=h(v) u$ (cf. (1.18)) with $h(v)>0$ (from (1.7) in section 1.1.3), $u_{s}<0$ implies $\dot{v}<0$ along $\gamma_{s}$.

LEMMA 5.2. Let us consider $S_{1}=S \cap N$ as in section 1.4.1.

- If $\alpha<1$, as shown by Figure 2 in section 1.4.1, $S_{1}$ is not accessible from $\left(z<d_{1}\right)$.

- If $\alpha>1$, any singular $B C$-extremal arriving on $N$ (with admissible singular control $u_{s}$ ) arrives at $S_{1}$ and is hyperbolic. Along $S, u_{s}$ relates biunivoquely and decreasingly the considered $v \in](\alpha \beta)^{\frac{1}{1-\alpha}}, A_{1}[$ onto $]-\infty, 0[;$ so there exists a single point of $S$ (denoted by $S_{\text {sat }}$ as in section 1.4.1) where $u_{s}=u_{-}$. And there is no exceptional singular $B C$-extremal arriving on $N$.

Proof. From the equation of $S$ given in Lemma 5.1, $S_{1}=\left(d_{1}, v_{1}\right)$ satisfies:

$$
1+d_{1}-\alpha \beta d_{1} v_{1}^{\alpha-1}=0
$$

So if $\alpha<1, S_{1} \notin A^{\prime}$ (defined in section 5.1.3) which contains the points of $N$ that are accessible from $\left(z<d_{1}\right)$. 
From Lemma 5.1, any singular $B C$-extremal arriving on $N$ arrives at $N \cap S=$ $S_{1}$; moreover its Hamiltonian is $H\left(S_{1}, p(0),\left.u_{s}\right|_{t=0}\right)=v_{1}+v_{1} d_{1}-\beta v_{1}^{\alpha} d_{1}$ (from (5.2), (5.3) in section 5.1.2) and so is positive when $\alpha>1$ from (5.6).

Then in that case $\alpha>1$, according to the definition recalled in section 2.1.4, $\gamma_{s}$ is hyperbolic if and only if $\left\langle p, \operatorname{ad}^{2} Y(X)\right\rangle<0$; and indeed, since $\gamma_{s}$ is included in $S$ (cf. Lemma 5.1), computations give:

$$
\left\langle p, \operatorname{ad}^{2} Y(X)\right\rangle=-\alpha(\alpha-1) \beta v^{\alpha-2} z h^{2}(v) p_{1}
$$

with $\alpha(\alpha-1) \beta>0$ (from section 1.1 and since $\alpha>1$ ), $v^{\alpha-2} z h^{2}(v)>0$ (from (1.6) and (1.7) in section 1.1.3) and $p_{1}>0$ (from section 5.1.2). So $\gamma_{s}$ is hyperbolic, with positive Hamiltonian (thus $\gamma_{s}$ is not exceptional).

Finally, according to Lemma 5.1, at any point $\left(z=\frac{1}{\alpha \beta v^{\alpha-1}-1}, v\right)$ of $S, u_{s}=-\frac{E_{1}}{\alpha R} \frac{v\left(\alpha \beta v^{\alpha-1}-1\right)}{\ln ^{2}\left(v / A_{1}\right)}$ is a function of $\left.v \in\right](\alpha \beta)^{\frac{1}{1-\alpha}}, A_{1}[$ (indeed $z>0$ and $v \in] 0, A_{1}[)$ such that $\lim _{v \rightarrow(\alpha \beta)^{\frac{1}{1-\alpha}}} u_{s}=0, \lim _{v \rightarrow A_{1}^{-}} u_{s}=-\infty$ and

$$
\frac{d}{d v}\left(u_{s}\right)=-\frac{E_{1}}{\alpha R \ell n^{3}\left(v / A_{1}\right)}\left[\left(\alpha^{2} \beta v^{\alpha-1}-1\right) \ln \left(v / A_{1}\right)-2\left(\alpha \beta v^{\alpha-1}-1\right)\right]
$$

so (since $v \in] 0, A_{1}\left[, \frac{E_{1}}{R}>0\right.$ and $\beta>0$ from section 1.1 and since $\alpha>1$ ),

$$
\frac{d}{d v}\left(u_{s}\right)<-\frac{E_{1}}{\alpha R \ell n^{3}\left(v / A_{1}\right)}\left(\alpha \beta v^{\alpha-1}-1\right)\left[\ln \left(v / A_{1}\right)-2\right]
$$

which has the sign of $-\left(\alpha \beta v^{\alpha-1}-1\right)<0$ since on $S, z=\frac{1}{\alpha \beta v^{\alpha-1}-1}$ is positive. So we get the result, specially for $S_{\text {sat }}$ since $u_{-}$is negative.

\subsubsection{Regular $B C$-extremals.}

LEMMA 5.3. - If $\alpha<1$, any point $\left(d_{1}, v \in\right] 0, A_{1}[)$ of $N$ such that $v>v_{A}$ $\left[v_{A}\right.$ is defined in section 5.1.3] is the arrival point of a $B C$-extremal with final control $u_{+}$.

- If $\alpha>1$, any point $\left(d_{1}, v \in\right] 0, A_{1}[)$ of $N$ such that $0<v<v_{1}$ (resp. $\left.v_{1}<v<v_{A}\right)\left[v_{1}\right.$ is defined in section 5.1.5] is the arrival point of a $B C$-extremal with final control $u_{+}$(resp. $u_{-}$).

Proof. Let $a=\left(d_{1}, v\right)$ be any point of $N$ belonging to $A^{\prime}$ with $v \neq v_{A}$ : then we know (from the proof of section 5.1.3) that for any $u_{0} \in\left[u_{-}, u_{+}\right]$, $H\left(a, p(0), u_{0}\right)>0$. And $\Phi(0)=0$ (cf. 5.1.4); so Corollary 2.2 implies that if $\dot{\Phi}(0) \neq 0$ there is only one $B C$-extremal arriving at $a$, the control being locally $u_{\varepsilon}$ with $\varepsilon=-\operatorname{sign}(\dot{\Phi}(0))$. Then comes the result according to the sign of $\alpha-1$, since $\dot{\Phi}(0)=-h(v)\left(1+d_{1}-\alpha \beta d_{1} v^{\alpha-1}\right)$ (cf. (5.3) in section 5.1.2 and (5.5) in section 5.1.4) and $h(v)>0$ (cf. (1.7) in section 1.1.3) with $v_{1}$ satisfying (5.6) in section 5.1.5.

LEMMA 5.4. If it exists, the point $G \cap N=\left(d_{1}, v_{A} \in\right] 0, A_{1}[)$ is not accessible from $\left(z<d_{1}\right)$. So $A=\left\{\left(d_{1}, v\right) \in A^{\prime} ; v \neq v_{A}\right\}, A^{\prime}$ and $v_{A}$ being defined in section 5.1.3. 
Proof. Let $a_{1}=\left(d_{1}, v_{A} \in\right] 0, A_{1}[)$ be $G \cap N$ if it exists. From the proof of section 5.1.3, $v_{A}$ is defined so that, for any $u_{0} \in\left[u_{-}, u_{+}\right], H\left(a_{1}, p(0), u_{0}\right)=0$.

And similarly as in Lemma 5.3 it turns out that, when arriving at $a_{1}$, the extremal control is locally $u_{\varepsilon}$ with $\varepsilon=-\operatorname{sign}(\dot{\Phi}(0))=\operatorname{sign}\left(1+d_{1}-\right.$ $\alpha \beta d_{1} v_{A}^{\alpha-1}$ ); thus, since (from section 5.1.3) $1+d_{1}=\beta d_{1} v_{A}^{\alpha-1}$ and since $1+d_{1}>0$, it comes $\varepsilon=\operatorname{sign}(1-\alpha)$.

But here we are in the exceptional case, so we have to check the position of the considered $\gamma_{\varepsilon}$ arc arriving at $a_{1}$ with respect to $N$ : indeed for this arc $z(0)=d_{1}, \dot{z}(0)=0$ (cf. section 5.1.3) and so the expansion of $z$ at time 0 to the second order implies that locally $\operatorname{sign}\left(z(t)-d_{1}\right)=\operatorname{sign}\left(z^{(2)}(0)\right)$. Moreover (1.18) (cf. section 1.4.1), with $u=u_{\varepsilon}, x(0)=\left(d_{1}, v_{A}\right)^{t}$ and so $\dot{z}(0)=0$, implies $z^{(2)}(0)=h\left(v_{A}\right) u_{\varepsilon}(1-\alpha)\left(1+d_{1}\right)$. So $z^{(2)}(0)>0$ since we know that $\varepsilon=\operatorname{sign}(1-\alpha)$, since $\varepsilon=\operatorname{sign}\left(u_{\varepsilon}\right)$ [indeed $u_{-}<0<u_{+}$was recalled in section 5.1.1] and since $h\left(v_{A}\right)>0$ from (1.7) in section 1.1.3. Finally we get for any time $t$ close enough to $0: z(t)-d_{1}>0$ and so comes the result.

\subsection{LOCAL SYNTHESIS}

In this section 5.2 we shall asume that $v_{A}<A_{1}\left[v_{A}\right.$ is the $v$-coordinate of $(\dot{z}=0) \cap N$ defined in section 5.1.3]; note that if this assumption does not hold (i.e. if $A_{1} \leq v_{A}$ ), the corresponding synthesis is simply obtained by restricting the following results to $\left(v<A_{1}\right)$.

5.2.1. CASE $\alpha<1$. Locally (near the target $N$ ), as shown by Figure 2 in section 1.4.1, the optimal trajectories are the $B C$-extremal trajectories arriving on $A=\left\{\left(d_{1}, v \in\right] 0, A_{1}[) \in N ; v>v_{A}\right\}$ which are $\gamma_{+}$arcs.

Proof. Lemmas 5.2 and 5.4 in the case $\alpha<1$ give all the $B C$-extremals, which are $\gamma_{+}$trajectories arriving on $A$.

\subsubsection{CASE $\alpha>1$. Locally (near the target $N$ ), the synthesis is shown by} Figure 15.

Proof. The synthesis near $G \cap N$ is formed by $\gamma_{-}$arcs according to Lemma 5.3. Since from Lemma 5.4, $G \cap N$ is not accessible from $\left(z<d_{1}\right)$, Figure 10 in section 5.3 of $[5]$ in the case $X_{2}(0)<1$ shows the corresponding local synthesis (indeed, it is the case where the exceptional trajectory is not admissible).

If $z_{\text {sat }} \geq d_{1}$, we know from Lemma 5.2 that $u_{s}<u_{-}$at any point of $S \cap\left(z<d_{1}\right)$ which is included in $S \cap\left(v>v_{\text {sat }}\right)$; so any singular arc, included in $S$ from Lemma 5.1, is parabolic (cf. section 2.3). The synthesis near $S_{1}$ is shown by Figure 15(i) according to Lemma 5.3 and to Figure 9 in section 5.2.4 of [5] in the case $a<0$ (indeed we know from section 5.2.1 of [5] that $a<0$ is the case where the singular arc arriving on $N$ is hyperbolic when the singular control is admissible, which is the considered case from Lemma 5.2 ).

If $z_{\text {sat }}<d_{1}$, we know from Lemma 5.2 that $u_{s} \leq u_{-}$at any point of $S \cap\left(z \leq z_{\text {sat }}\right)=S \cap\left(v \geq v_{\text {sat }}\right)$, that $\left.u_{s} \in\right] u_{-}, 0[\subset] u_{-}, u_{+}$[ at any point of $S \cap\left(z_{\text {sat }}<z \leq d_{1}\right)=S \cap\left(v_{1} \leq v<v_{\text {sat }}\right)$ and that any singular arc included 


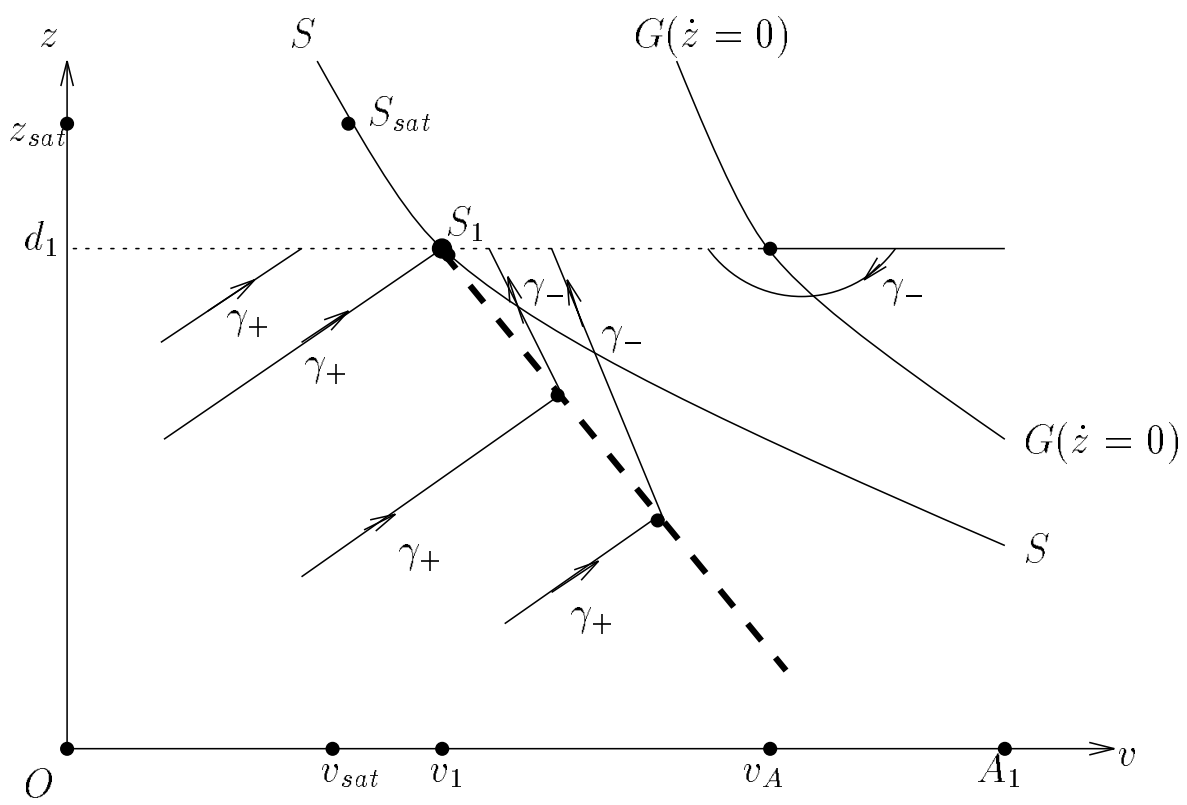

Figure $15(\mathrm{i}): z_{\text {sat }} \geq d_{1}$

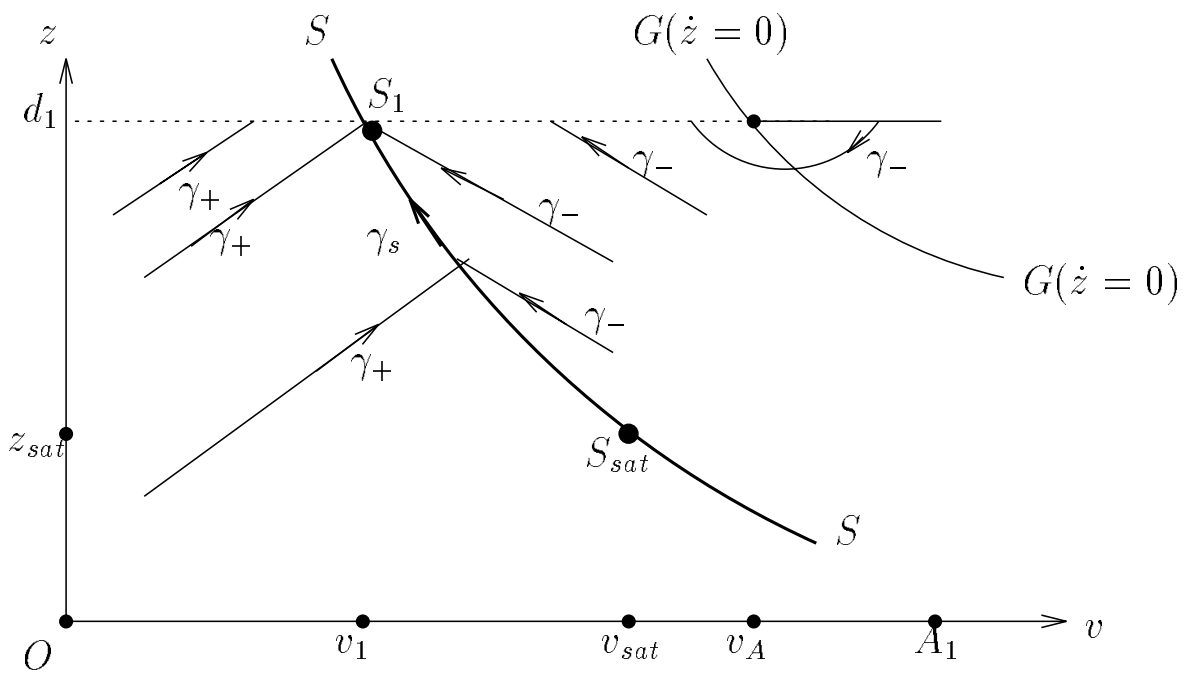

Figure 15(ii) : $z_{\text {sat }}<d_{1}$

A, _ _ - set of switching points for $\gamma_{+} \gamma_{-}$

Figure 15

in the arc $\left(S_{\mathrm{sat}} S_{1}\right)$ of $S$ is hyperbolic. The synthesis near $S_{1}$ is thus shown by Figure 15 (ii) according to section 5.2 .2 of [5]. 


\subsection{Global switching Results in the case $\alpha>1$}

5.3.1. Switching ReSUlt FOR $\gamma_{+}$EXTRemal arCs. Let $\gamma_{+}$, defined for times $t \in\left[t_{1}, t_{2}\right]$, be a non-empty subarc of a $B C$-extremal trajectory; then, if $t_{2}$ is a switching time, $t_{1}$ cannot be a switching time.

Proof. From section 2.1.3 $\gamma_{+}$satisfies $\Phi(t)>0$ at any time $\left.t \in\right] t_{1}, t_{2}[$; moreover, if $t_{1}$ and $t_{2}$ are switching times, $\Phi\left(t_{1}\right)=\Phi\left(t_{2}\right)=0$ (cf. Corollary 2.2), and so we should have $\dot{\Phi}\left(t_{1}\right) \geq 0$ and $\dot{\Phi}\left(t_{2}\right) \leq 0$.

Let us consider $\psi=\dot{\Phi} / h(v)$. Then, since $h(v)>0$ (cf. (1.7) in section 1.1.3), we should have $\psi\left(t_{1}\right) \geq 0$ and $\psi\left(t_{2}\right) \leq 0$. And computations taking into account (5.5) in section 5.1 .4 and (1.18) in section 1.4 .1 yield $\dot{\psi}=$ $(\alpha-1) \beta v^{\alpha-2}\left[v^{2}+\alpha z h(v) u_{+}\right] p_{1} ;$ so, since $(\alpha-1) \beta>0$ (from section 1.1 and since $\alpha>1$ ), $v>0, z \geq 0, h(v)>0, u_{+}>0$ (from section 1.1) and $p_{1}>0$ (from section 5.1.2), it comes $\dot{\psi}>0$ along $\gamma_{+}$: this is contradictory with $\psi\left(t_{1}\right) \geq 0$ and $\psi\left(t_{2}\right) \leq 0$ knowing that $t_{1}<t_{2}$.

5.3.2. Optimal trajectories. Optimal trajectories are shown by Figure 3 in section 1.4.1.

Indeed, $z_{\text {sat }}$ denoting the z-coordinate of the unique point $S_{\text {sat }}$ of $S$ where $u_{s}=u_{-}$(cf. section 5.1.5), optimal trajectories are:

- if $z_{\text {sat }} \geq d_{1}: \gamma_{+} \gamma_{-}$(see Figure $3(i)$ )

- if $z_{\text {sat }}<d_{1}: \gamma_{+} \gamma_{-} \gamma_{s}$ (see Figure $3(i i)$ )

where each arc of these sequences may be empty.

Notice that if $z_{\text {sat }} \geq d_{1}$, the optimal policy is just an extension of the local synthesis described in section 5.2.2; whereas if $z_{\text {sat }}<d_{1}$ a supplementary switch may occur for $\gamma_{-}$arcs when globalizing.

Proof. • If $z_{\text {sat }} \geq d_{1}$, sections 5.2.2 and 5.3.1 imply that any optimal trajectory is $\gamma_{+} \gamma_{-}$(where each arc of this sequence may be empty) and that these optimal trajectories are shown by Figure 3(i).

- If $z_{\text {sat }}<d_{1}$, from sections 5.2.2, 5.3.1 and section 6.2 .7 of [5], a sufficient condition for the result is that no sequence $\gamma_{s} \gamma_{-}$(with $\gamma_{s}$ and $\gamma_{-}$ non-empty) can appear in a $B C$-extremal trajectory.

Indeed in that case $z_{\text {sat }}<d_{1}$, if there exists an extremal sequence $\gamma_{s} \gamma_{-}$ (with $\gamma_{s}$ and $\gamma_{-}$non-empty), let us denote by $\sigma=\left(z_{\sigma}, v_{\sigma}\right)$ the switching point from $\gamma_{s}$ to $\gamma_{-}$and $t_{\sigma}$ the corresponding switching time. Then, as shown by Figure 16:

the point $\sigma$ belongs necessarily to the open arc $\left(S_{\mathrm{sat}} S_{1}\right)$ of $S$ from Lemma 5.2, with $\sigma \neq S_{1}\left(\operatorname{resp} . S_{\text {sat }}\right)$ for $\gamma_{-}\left(\operatorname{resp} . \gamma_{s}\right)$ not to be empty; so $\left.\left.u_{s}\right|_{x=\sigma} \in\right] u_{-}, 0[$; thus from (1.18) in section 1.4.1, it comes for the extremal sequence $\gamma_{s} \gamma_{-}$ at the switching time $t_{\sigma}: \lim _{t \rightarrow t_{\sigma}^{+}} \dot{v}(t)=h\left(v_{\sigma}\right) u_{-}<\lim _{t \rightarrow t_{\sigma}^{-}} \dot{v}(t)=\left.h\left(v_{\sigma}\right) u_{s}\right|_{x=\sigma}$ while $\dot{z}$ is continuous at time $t_{\sigma}$; so at any time $t>t_{\sigma}$ close enough to $t_{\sigma}$, the arc $\gamma_{-}$starting from point $\sigma$ is on the left of $S$;

but from section 5.2.2 and section 6.2 .7 of [5], the arc $\left(S_{\mathrm{sat}} S_{1}\right)$ of $S$ separates optimal arcs $\gamma_{+}$(on the left of $S$ ) and $\gamma_{-}$(on the right of $S$ );

so no extremal arc $\gamma_{-}$can be found on the left of $S$ near $\sigma$.

Thus, optimal trajectories are shown by Figure 3(ii). 


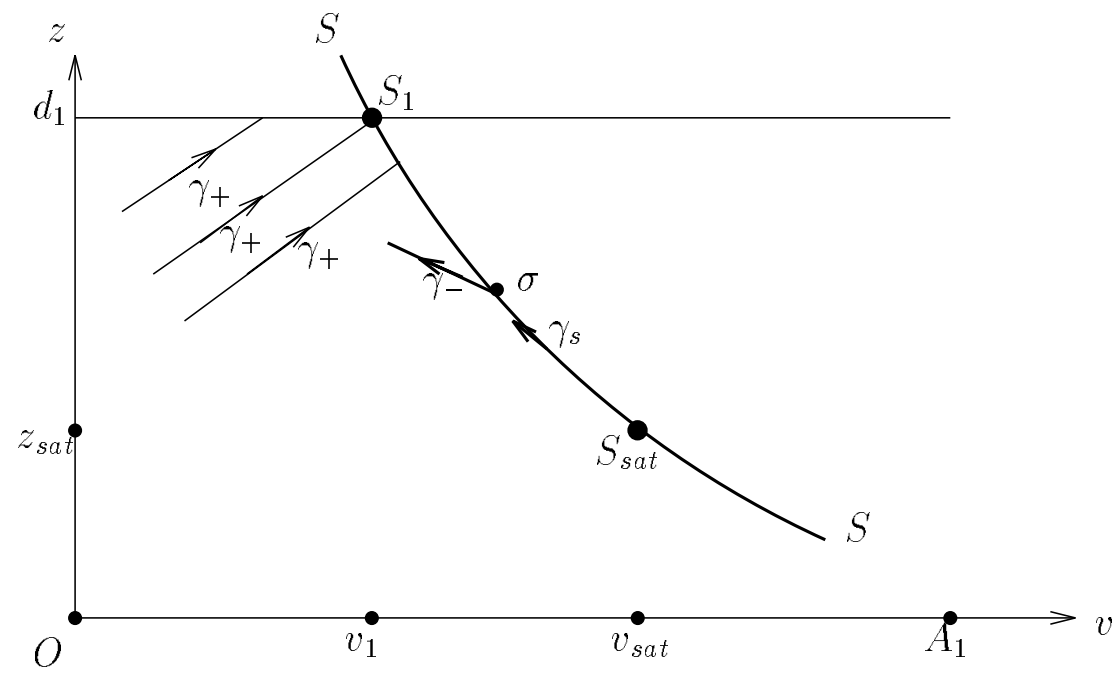

Figure 16

\section{Time Minimal SyNThesis FOR $\dot{\mathcal{P}}_{2}$}

\subsection{Generalities}

6.1.1. Definitions and notations. According to section 3.1.3, we can consider instead of $\dot{\mathcal{P}}_{2}$ the equivalent time minimal projected problem $\dot{\mathcal{P}}_{2}^{\prime}$ with system (3.5), control $u \in\left[u_{-}, u_{+}\right]$with $u_{-}<0<u_{+}$and terminal manifold $N:\left(c_{2}=d_{2}\right)$ to be reached from an initial value $\left(c_{2}\right)_{0} \in\left[0, d_{2}\right.$. So the state-vector is $x=\left(x_{1}, c_{2}, v\right)^{t}, x_{1}$ being related to the original variable $c_{1}$ by (3.2). Note that if $n_{1}>1$ it comes $x_{1}<0$ since (cf. (1.6) in section 1.1.3) $c_{1}>0$.

Let us denote by $u_{s}$ the singular control and by $\gamma_{-}\left(\operatorname{resp} . \gamma_{+}, \gamma_{s}\right)$ any arc satisfying (3.5) with control $u_{-}$(resp. $u_{+}, u_{s}$ ).

6.1.2. Adjoint state. Along any $B C$-extremal $(x, p, u)$, the adjoint vector $p=\left(p_{1}, p_{2}, p_{3}\right)^{t}$ associated to $x=\left(x_{1}, c_{2}, v\right)^{t}$ satisfies (when taking (3.2) into account):

$$
\left\{\begin{array}{l}
\dot{p}_{1}=-n_{1} v c_{1}^{2 n_{1}-1} p_{2} \\
\dot{p}_{2}=n_{2} \beta v^{\alpha} c_{2}^{n_{2}-1} p_{2} \\
\dot{p}_{3}=p_{1}+\left(-c_{1}^{n_{1}}+\alpha \beta v^{\alpha-1} c_{2}^{n_{2}}\right) p_{2}-\frac{d h}{d v}(v) u p_{3}
\end{array}\right.
$$

the corresponding Hamiltonian being:

$$
H(x, p, u)=-v p_{1}+\left(v c_{1}^{n_{1}}-\beta v^{\alpha} c_{2}^{n_{2}}\right) p_{2}+h(v) u p_{3}
$$

At final time 0 , we can take

$$
p(0)=(0,1,0)
$$

and at any negative time $t, p_{1}(t)>0$ and $p_{2}(t)>0$.

Proof. Similar to the proof of section 4.1.2. 
6.1.3. Accessibility. Let $A$ be the set of points of the target $N:\left(c_{2}=d_{2}\right)$ that are accessible from $\left(c_{2}<d_{2}\right)$. Then $A$ is included in $A^{\prime}=\left\{\left(c_{1}>\right.\right.$ $\left.\left.0, d_{2}, v \in\right] 0, A_{1}[) \in N ; c_{1}^{n_{1}}-\beta v^{\alpha-1} d_{2}^{n_{2}} \geq 0\right\}$, the frontier of which is $\mathcal{E}=$ $\left\{\left(c_{1}>0, d_{2}, v \in\right] 0, A_{1}[) \in N ; c_{1}^{n_{1}}-\beta v^{\alpha-1} d_{2}^{n_{2}}=0\right.$ i.e. $\left.\dot{c}_{2}=0\right\}$.

Proof. For any $u_{0} \in\left[u_{-}, u_{+}\right]$any point $a=\left(c_{1}, d_{2}, v\right)$ of $A$ reached with final control $u_{0}$ has to satisfy $H\left(a, p(0), u_{0}\right) \geq 0$ i.e., from $(6.2),(6.3)$ in section $6.1 .2 v c_{1}^{n_{1}}-\beta v^{\alpha} d_{2}^{n_{2}} \geq 0$, and so comes the result since $v>0$ from (1.6) in section 1.1.3.

6.1.4. SWitching FUnCtion. The switching function of $\dot{\mathcal{P}}_{2}^{\prime}$ is

$$
\Phi: t \mapsto h(v(t)) p_{3}(t)
$$

So (1.7) in section 1.1.3 implies that

$$
\Phi(t)=0 \Leftrightarrow p_{3}(t)=0
$$

And at time $0, \Phi(0)=0$ (cf. (6.3) in section 6.1.2). Indeed we can notice that problem $\dot{\mathcal{P}}_{2}^{\prime}$ is flat.

\section{Moreover}

$$
\dot{\Phi}=\left[p_{1}+\left(-c_{1}^{n_{1}}+\alpha \beta v^{\alpha-1} c_{2}^{n_{2}}\right) p_{2}\right] h(v)
$$

Proof. Computations, applying equations (2.2), (2.3) of section 2.1 .2 to problem $\dot{\mathcal{P}}_{2}^{\prime}$ stated in section 6.1 .1 with affine single-input system (3.5).

\subsection{Stratification of the target $N$}

\subsubsection{Singular $B C$-extremals.}

Lemma 6.1. As in section 1.4.3, let $S_{2}$ be the set of points $\left(c_{1}>0, d_{2}, v \in\right.$ ]0, $A_{1}[)$ of $N$ such that $\frac{d}{d t} \frac{\partial H}{\partial u}=0$. Then $S_{2}=\left\{\left(c_{1}>0, d_{2}, v \in\right] 0, A_{1}[) \in\right.$ $\left.N ; c_{1}^{n_{1}}-\alpha \beta v^{\alpha-1} d_{2}^{n_{2}}=0\right\}$. And any singular $B C$-extremal arriving on $N$ arrives on $S_{2}$. So, when $\alpha<1$, since $S_{2} \cap A^{\prime}=\emptyset$, there is no singular $B C$-extremal arriving on $N$.

Proof. From section 1.3.4 any singular $B C$-extremal has to satisfy $\frac{d}{d t} \frac{\partial H}{\partial u}=$ 0 , so any singular $B C$-extremal arriving on $N$ arrives on $S_{2}$.

From (6.2) in section $6.1 .2, \frac{d}{d t} \frac{\partial H}{\partial u}=0$ is equivalent to $\frac{d}{d t}\left(h(v) p_{3}\right)=0$ which is equivalent from (3.5) in section 3.1 .3 and $(6.1)$ in section 6.1 .2 to $h(v)\left[c_{1}^{n_{1}} p_{1}-\left(c_{1}^{n_{1}}-\alpha \beta v^{\alpha-1} c_{2}^{n_{2}}\right) p_{2}\right]=0$. We thus find the expected result from (1.7) in section 1.1.3, (6.3) in section 6.1 .2 and $c_{2}(0)=d_{2}$ since we assume that the considered trajectory arrives on $N$ at final time 0 .

Finally, when $\alpha<1$, any point $\left(c_{1}, d_{2}, v\right)$ of $S_{2}$ satisfies $c_{1}^{n_{1}}-\beta v^{\alpha-1} d_{2}^{n_{2}}<0$ and so $S_{2} \cap A^{\prime}=\emptyset$ : this implies that $S_{2} \cap A=\emptyset\left(A \subset A^{\prime}\right.$ was proved in section 6.1.3) and thus there is no singular $B C$-extremal arriving on $N$. 
Lemma 6.2. Let us assume that $\alpha>1$ and $c_{2}>0$. Along any singular $B C$-extremal arriving on $N$ the singular control is:

$$
u_{s}=-\frac{n_{2}}{\alpha} \frac{c_{1}^{n_{1}} v^{2}}{c_{2} h(v)}<0
$$

and specially at the arrival point $x(0) \in S_{2}$, the final singular control is:

$$
\left.u_{s}\right|_{t=0}=-n_{2} \beta d_{2}^{n_{2}-1} \frac{v(0)^{\alpha+1}}{h(v(0))}<0
$$

Then $\left.v(0) \mapsto u_{s}\right|_{t=0}$ relates biunivoquely and decreasingly $] 0, A_{1}[$ onto ]$-\infty, 0\left[;\right.$ so there exists a single point of $S_{2}$ (denoted by $S_{\text {sat }}$ as in section 1.4.3) where $\left.u_{s}\right|_{t=0}=u_{-}$.

Proof. Equation (6.7) comes from section 2.4 applied to problem $\dot{\mathcal{P}}_{2}^{\prime}$ with affine single-input system (3.5): indeed we find

$$
\left\{\begin{array}{l}
D=\alpha(\alpha-1) \beta c_{2}^{n_{2}} v^{\alpha-2} h^{4}(v) \\
D^{\prime}=(\alpha-1) \beta n_{2} c_{1}^{n_{1}} c_{2}^{n_{2}-1} v^{\alpha} h^{3}(v) \\
D^{\prime \prime}=(\alpha-1) \beta c_{2}^{n_{2}} v^{\alpha} h^{2}(v)
\end{array}\right.
$$

Since $\beta>0$ from section $1.1, c_{1}>0$ and $v>0$ from (1.6) in section 1.1.3, $h(v)>0$ from (1.7) in section 1.1 .3 and since we assume that $\alpha>1$ and $c_{2}>0$, it comes $D \neq 0$ and then (6.7) when applying section 2.4 again.

Equation (6.8) comes from (6.7) considered at time 0 , knowing from Lemma 6.2 that $x(0) \in S_{2}$.

Then from the value of $h(v)$ (cf. (3.5) in section 3.1.3) and from (6.8) we get

$$
\left.u_{s}\right|_{t=0}=-\frac{n_{2} E_{1} \beta}{R} d_{2}^{n_{2}-1} \frac{v(0)^{\alpha}}{\ln ^{2}\left(v(0) / A_{1}\right)}
$$

which is a function of $v(0) \in] 0, A_{1}[$ such that

$$
\left.\lim _{v(0) \rightarrow 0^{+}} u_{s}\right|_{t=0}=0,\left.\quad \lim _{v(0) \rightarrow A_{1}^{-}} u_{s}\right|_{t=0}=-\infty
$$

and

$$
\frac{d}{d v(0)}\left(\left.u_{s}\right|_{t=0}\right)=-\frac{n_{2} E_{1} \beta}{R} d_{2}^{n_{2}-1} \frac{\left[\alpha \ln \left(v(0) / A_{1}\right)-2\right] v^{\alpha-1}(0)}{\ln ^{3}\left(v(0) / A_{1}\right)}<0
$$

since $v \in] 0, A_{1}\left[, \frac{E_{1} \beta}{R}>0\right.$ and $d_{2}>0$ from section 1.1 . So we get the result, specially for $S_{\text {sat }}$ since $u_{-}$is negative.

LeMMA 6.3. Let us assume that $\alpha>1$ and $c_{2}>0$.

Along the singular arc (denoted by $\gamma_{\text {sat }}$ ) arriving at $S_{\text {sat }}, u_{s}<u_{-}$at any negative time close enough to $0:$ so $\gamma_{\text {sat }}$ is not extremal.

Any singular $B C$-extremal arriving on $N$ (with admissible singular control $\left.u_{s}\right)$ is hyperbolic and arrives at a point $\left(c_{1}=\alpha \beta v^{\alpha-1} d_{2}, d_{2}, v\right)$ of $S_{2}$ such that $v<v_{\text {sat }}$, where $v_{\text {sat }}$ denotes the $v$-coordinate of $S_{\text {sat }}$.

Any point $\left(c_{1}=\alpha \beta v^{\alpha-1} d_{2}, d_{2}, v\right)$ of $S_{2}$ such that $v>v_{\text {sat }}$ is a parabolic fold point. 
Proof. From (6.9) it comes $D D^{\prime \prime}=\alpha(\alpha-1)^{2} \beta^{2} c_{2}^{2 n_{2}} v^{2 \alpha-2} h^{6}(v)$ which implies that $D D^{\prime \prime}>0$ since $\left.v \in\right] 0, A_{1}[$ and $\beta>0$ from section $1.1, h(v)>0$ from (1.7) in section 1.1.3 and since we assume that $\alpha>1$ and $c_{2}>0$.

Then we know from Corollary 2.6 and from section 2.3 that any point of $S_{2}$ is an hyperbolic (resp. parabolic) fold point if $\left.\left.u_{s}\right|_{t=0} \in\right] u_{-}, u_{+}$[ (resp. $\left.\left.u_{s}\right|_{t=0} \notin\left[u_{-}, u_{+}\right]\right)$.

So Lemma 6.2 yields the result claimed about points of $S_{2}$ that are not $S_{\text {sat }}$.

The singular arc $\gamma_{\text {sat }}$ arrives at $S_{\text {sat }}$ with saturated final control $\left.u_{s}\right|_{t=0}=$ $u_{-}$: the local synthesis near such points is described in section 6.2.7 of [5]. Notice that in the case where singular arcs arriving on $N$ are hyperbolic when the singular control is admissible (cf. sections 6.2 .3 and 6.2 .4 of [5]), which is just proved to be considered case, the study of the synthesis splits into two subcases, depending on the admissibility of the singular control along the trajectory that arrives at the saturation point; to find out about it, let us study the sign of $\left\langle X\left(S_{\mathrm{sat}}\right)+u_{-} Y\left(S_{\mathrm{sat}}\right),\left(\nabla_{x} u_{s}\right)\left(S_{\mathrm{sat}}\right)\right\rangle$ : it appears that if this sign is plus $u_{s}<u_{-}$along $\gamma_{\text {sat }}$ at any negative time close enough to 0 .

Indeed at any point $\left(c_{1}>0, c_{2}>0, v \in\right] 0, A_{1}[)$, with $x=\left(x_{1}, c_{2}, v\right)^{t}$ and $x_{1}$ related to $c_{1}$ by $(3.2)$ (cf. section 3.1 .3 ), it comes:

$$
\begin{aligned}
& \left\langle X(x)+u_{-} Y(x), \nabla_{x} u_{s}\right\rangle=\frac{n_{2}}{\alpha}\left(n_{1} \frac{c_{1}^{2 n_{1}-1}}{c_{2}}+\frac{c_{1}^{2 n_{1}}}{c_{2}^{2}}\right) \frac{v^{3}}{h(v)}- \\
& \frac{n_{2}}{\alpha}\left[\beta c_{1}^{n_{1}} c_{2}^{n_{2}-2} \frac{v^{\alpha+2}}{h(v)}+u_{-} \frac{c_{1}^{n_{1}}}{c_{2}} v\left(1-\frac{2}{\ln \left(v / A_{1}\right)}\right)\right]
\end{aligned}
$$

So for $x=S_{\text {sat }}$ such that $c_{2}=d_{2}$ (since $\left.S_{\text {sat }} \in N\right), c_{1}^{n_{1}}=\alpha \beta d_{2}^{n_{2}} v^{\alpha-1}$ (since $S_{\mathrm{sat}} \in S_{2}$ and from Lemma 6.1$)$ and $-n_{2} \beta d_{2}^{n_{2}-1} \frac{v^{\alpha+1}}{h(v)}=u_{-}$(since $u_{s}=u_{-}$ at $S_{\text {sat }}$ and from (6.8) in section 6.2.1) it comes:

$$
\begin{aligned}
& \left\langle X\left(S_{\mathrm{sat}}\right)+u_{-} Y\left(S_{\mathrm{sat}}\right),\left(\nabla_{x} u_{)}\left(S_{\mathrm{sat}}\right)\right\rangle=\right. \\
& -\frac{n_{2} u_{-} v}{\alpha}\left[\frac{n_{1}}{n_{2} \beta} \frac{c_{1}^{2 n_{1}-1} v^{1-\alpha}}{d_{2}^{n_{2}}}+\frac{(\alpha-1) c_{1}^{n_{1}}}{n_{2} d_{2}}+\frac{c_{1}^{n_{1}}}{d_{2}}\left(1-\frac{2}{\ln \left(v / A_{1}\right)}\right)\right]
\end{aligned}
$$

which is positive since $\left.c_{1}>0, d_{2}>0, v \in\right] 0, A_{1}\left[, u_{-}<0\right.$ and $\beta>0$ from section 1.1 and since we are in the case $\alpha>1$.

6.2.2. Regular BC-EXtremals. Any point of $A^{\prime} \backslash \mathcal{E}$ strictly above (resp. under) $S_{2}$ is the arrival point of a $B C$-extremal with final control $u_{+}$(resp $\left.u_{-}\right)$.

Proof. Let $a=\left(c_{1}, d_{2}, v\right)$ be any point of $A^{\prime} \backslash \mathcal{E}$. Then we know (from the proof of section 6.1.3) that for any $u_{0} \in\left[u_{-}, u_{+}\right] H\left(a, p(0), u_{0}\right)>0$. And $\Phi(0)=0$ (from section 6.1.4); so Corollary 2.2 implies that if $\dot{\Phi}(0) \neq 0$ there is only one $B C$-extremal arriving at $a$, the control being locally $u_{\varepsilon}$ with $\varepsilon=-\operatorname{sign}(\dot{\Phi}(0))$. Then comes the result, from the value of $\dot{\Phi}(0)$ (cf. (6.3) in section 6.1 .2 and $(6.6)$ in section 6.1 .4 ) and from the equation of $S_{2}$ (cf. Lemma 6.1). 


\subsubsection{EXcEPTIONAL CASE.}

LEMMA 6.4. Let $E$ be the exceptional locus, defined as the set of final points of BC-extremal trajectories tangent to $N$. Then $E$ is included in $\mathcal{E}$ (defined in section 6.1.3 as the frontier of $A^{\prime}$ ). If $\alpha<1$ (resp. $\alpha>1$ ) the final control on $E$ is $u_{+}$(resp. $u_{-}$). And as $S_{2} \cap \mathcal{E}=\emptyset$ there is no singular exceptional $B C$-extremal arriving on $N$.

Proof. For a $B C$-extremal $(x, p, u)$, we are in the exceptional case if and only if the Hamiltonian $H$ is zero, i.e. (from PMP) if and only if $\left.H\right|_{t=0}=0$ which from $(6.2)$ and $(6.3)$ in section 6.1 .2 is equivalent to $v(0) c_{1}^{n_{1}}(0)-\beta v^{\alpha}(0) d_{2}^{n_{2}}=$ 0 : thus the arrival point $x(0)$ belongs to $\mathcal{E}$ defined in section 6.1 .3 since $v(0) \in] 0, A_{1}[$ is not zero.

Similarly as in section 6.2 .2 , it turns out that, when arriving on $E$, the extremal control is locally $u_{\varepsilon}$ with $\varepsilon=-\operatorname{sign}(\dot{\Phi}(0))=+$ (resp. - ) if $\alpha<1$ (resp. $\alpha>1$ ) since $S_{2}$ is under (resp. above) $\mathcal{E}$.

$S_{2} \cap \mathcal{E}=\emptyset$ from equations of $\mathcal{E}$ (cf. section 6.1.3) and $S_{2}$ (cf. Lemma 6.1). So comes the result since we just proved that any exceptional $B C$ extremal arriving on $N$ arrives on $\mathcal{E}$ and since from Lemma 6.1 any singular $B C$-extremal arriving on $N$ arrives on $S_{2}$.

LeMMa 6.5. There exists a single point of $\mathcal{E}$ [denoted as in section 1.4.3 $\mathcal{E}_{+}$(resp. $\mathcal{E}_{-}$) when $\alpha<1$ (resp. $\alpha>1$ )] such that the exceptional arc arriving at this point has with $N$ a contact of order greater than two; and the exceptional locus $E$ is the set of points of $\mathcal{E}$ such that $v_{+}<v<A_{1}$ (resp. $\left.v_{-} \leq v<A_{1}\right)$, where $v_{+}$(resp. $v_{-}$) denotes the $v$-coordinate of $\mathcal{E}_{+}$(resp. $\left.\mathcal{E}_{-}\right)$.

Proof. At any point $a=\left(c_{1}, d_{2}, v\right)$ of $\mathcal{E}$, we have $\left.\dot{c}_{2}\right|_{x=a}=0$ i.e. $c_{1}^{n_{1}}=$ $\beta v^{\alpha-1} d_{2}^{n_{2}}$ (cf. section 6.1.3). And from Lemma 6.4, if a $B C$-extremal arrives at $a$, the final control is $u_{\varepsilon}$ with $\varepsilon=\operatorname{sign}(1-\alpha)$. Thus at final time 0 (i.e. at point $a$ ) it comes from (3.5) in section 3.1.3:

$$
\begin{aligned}
& c_{2}^{(2)}(0)=\beta d_{2}^{n_{2}} v^{\alpha} \Gamma(v) \text { with } \\
& \Gamma(v)=(1-\alpha) u_{\varepsilon} \frac{R}{E_{1}} \ln ^{2}\left(\frac{v}{A_{1}}\right)-n_{1} \beta^{1-\frac{1}{n_{1}}} d_{2}^{n_{2}\left(1-\frac{1}{n_{1}}\right)} v^{\alpha+\frac{1}{n_{1}}(1-\alpha)} \text {. Then, since } \\
& \left.\frac{E_{1}}{R}>0, \alpha>0, \beta>0, v \in\right] 0, A_{1}[\text { from section } 1.1 \text { and since } \\
& (1-\alpha) u_{\varepsilon}>0, \\
& \lim _{v \rightarrow 0^{+}} \Gamma(v)=+\infty, \\
& \lim _{v \rightarrow A_{1}^{-}} \Gamma(v)=-n_{1} \beta^{1-\frac{1}{n_{1}}} d_{2}^{n_{2}\left(1-\frac{1}{n_{1}}\right)} A_{1}^{\alpha+\frac{1}{n_{1}}(1-\alpha)}<0 \text { and } \\
& \frac{d}{d v} \Gamma(v)=2(1-\alpha) u_{\varepsilon} \frac{R}{E_{1} v} \ln \left(\frac{v}{A_{1}}\right) \\
& \quad-n_{1} \beta^{1-\frac{1}{n_{1}}} d_{2}^{n_{2}\left(1-\frac{1}{n_{1}}\right)}\left(\frac{1}{n_{1}}+\alpha\left(1-\frac{1}{n_{1}}\right)\right) v^{(\alpha-1)\left(1-\frac{1}{n_{1}}\right)}<0 .
\end{aligned}
$$

So there exists a single value $v_{\varepsilon}$ of $\left.v \in\right] 0, A_{1}\left[\right.$ such that $c_{2}^{(2)}(0)=0$; and for any $v \in] 0, v_{\varepsilon}[$ (resp. $] v_{\varepsilon}, A_{1}[) c_{2}^{(2)}(0)>0$ (resp. $\left.c_{2}^{(2)}(0)<0\right)$ : thus the $\gamma_{\varepsilon}$ arc arriving at $a$ is locally in $\left(c_{2}>d_{2}\right)$ (resp. in $\left(c_{2}<d_{2}\right)$ ) from the expansion ESAIM: CoCv, DeCEMBer 1998, Vol. 3, 407-467 
of $c_{2}$ at time 0 to the second order with $c_{2}(0)=d_{2}$ and $\dot{c}_{2}(0)=0$, and so comes the result at any point $a$ of $\mathcal{E}$ such that $a \neq \mathcal{E}_{\varepsilon}$.

And for $a=\mathcal{E}_{\varepsilon}$, the expansion of $c_{2}$ at time 0 to the third order with $c_{2}(0)=d_{2}$ and $\dot{c}_{2}(0)=c_{2}^{(2)}(0)=0$ implies that at any negative time $t$ close enough to $0 \operatorname{sign}\left(c_{2}(t)-d_{2}\right)=-\operatorname{sign}\left(c_{2}^{(3)}(0)\right)$. Computations give in this case $c_{2}^{(3)}(0)=\beta u_{\varepsilon} d_{2}^{n_{2}} v^{\alpha} h(v) \frac{d}{d v} \Gamma(v)$ and so, since $\left.\beta>0, \operatorname{sign}\left(u_{\varepsilon}\right)=\varepsilon, v \in\right] 0, A_{1}[$ from section 1.1, $h(v)>0$ from (1.7) in section 1.1.3 and since $\frac{d}{d v} \Gamma(v)<0$, $\operatorname{sign}\left(c_{2}(t)-d_{2}\right)=\varepsilon$. Thus, since $\varepsilon=\operatorname{sign}(1-\alpha)$, if $\alpha<1(\operatorname{resp} . \alpha>1)$ the $\gamma_{\varepsilon}$ arc arriving at $\mathcal{E}_{\varepsilon}$ is locally included in $\left(c_{2}>d_{2}\right)$ (resp. $\left(c_{2}<d_{2}\right)$ ).

6.2.4. Stratification of $N$. Figure 17 summarizes the previous results about final control on the target $N$, where $\bar{A}^{\prime}$ denotes the complementary of $A^{\prime}$ in the physical domain $\left(c_{1}>0, v \in\right] 0, A_{1}[)$ of $N$.

\subsection{LOCAL SYNTHESIS}

6.3.1. CASE $\alpha<1$. Locally (near the target $N$ ), the synthesis is shown by Figure 7 in section 1.4 .3

Proof. Lemma 6.1, sections 6.2.2 and 6.2.3 give all the $B C$-extremals, which are $\gamma_{+}$trajectories arriving on $A=\left(A^{\prime} \backslash \mathcal{E}\right) \cup\left\{\left(c_{1}>0, d_{2}, v\right) \in \mathcal{E} ; v_{\mathcal{E}_{+}}<v<\right.$ $\left.A_{1}\right\}$. Since $\mathcal{E}_{+}$is not accessible from $\left(c_{2}<d_{2}\right)$ (cf. Lemma 6.5 ) we have to refer to Figure 8 in section 3 of [14]

6.3.2. CASE $\alpha>1$. Locally (near the target $N$ ), the synthesis is shown by Figures 8 and 9 in section 1.4.3.

Proof. As noticed in the proof of Lemma 6.3, the local synthesis near $S_{2}$ is described in section 6.2 .7 of [5] and specially is shown by Figure 17 since the singular control is not admissible along the trajectory that arrives at the saturation point. So the synthesis near $S_{2}$ is shown by Figure 8 in section 1.4.3.

Since (according to Lemma 6.5$) \mathcal{E}_{-}$is accessible from $\left(c_{2}<d_{2}\right)$ we have to refer to Figure 5 in section 3 of [14]; and since the synthesis near $\mathcal{E}$ is formed of $\gamma_{-}$arcs (cf. Lemma 6.4 ), it is shown by Figure 9 in section 1.4.3.

\subsection{Global switching Results in the case $\alpha>1$}

6.4.1. Switching ReSUlt FOR $\gamma_{+}$EXTREMAL ARCS. Let $\gamma_{+}$, defined for times $t \in\left[t_{1}, t_{2}\right]$, be a non-empty subarc of a $B C$-extremal trajectory; then, if $t_{2}$ is a switching time, $t_{1}$ cannot be a switching time.

Proof. From section 2.1.3, $\gamma_{+}$satisfies $\Phi(t)>0$ at any time $\left.t \in\right] t_{1}, t_{2}[$; moreover, if $t_{1}$ and $t_{2}$ are switching times, $\Phi\left(t_{1}\right)=\Phi\left(t_{2}\right)=0$ (cf. Corollary 2.2 ), and so we should have $\dot{\Phi}\left(t_{1}\right) \geq 0$ and $\dot{\Phi}\left(t_{2}\right) \leq 0$.

Let us consider $\psi=\dot{\Phi} / h(v)$. Then, from (1.7) in section 1.1.3, we should have $\psi\left(t_{1}\right) \geq 0$ and $\psi\left(t_{2}\right) \leq 0$. And computations taking into account $(6.4)$ and $(6.6)$ in section $6.1 .4,(3.5)$ in section 3.1 .3 and $(6.1)$ in section 6.1 .2 yield $\psi=p_{1}-c_{1}^{n_{1}} p_{2}+\alpha \beta c_{2}^{n_{2}} v^{\alpha-1} p_{2}$, and:

$$
\dot{\psi}=(\alpha-1) \beta\left[n_{2} c_{1}^{n_{1}} c_{2}^{n_{2}-1} v^{\alpha}+\alpha u c_{2}^{n_{2}} v^{\alpha-2} h(v)\right] p_{2}
$$




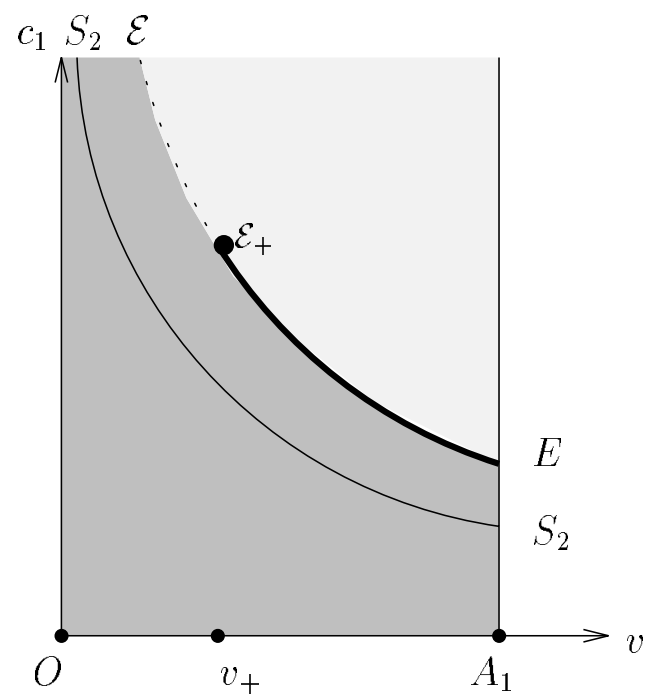

Figure 17 (i) : $\alpha<1$

non-accessible points:

and on $\mathcal{E}$, with $\mathcal{E}_{+}$non-accessible

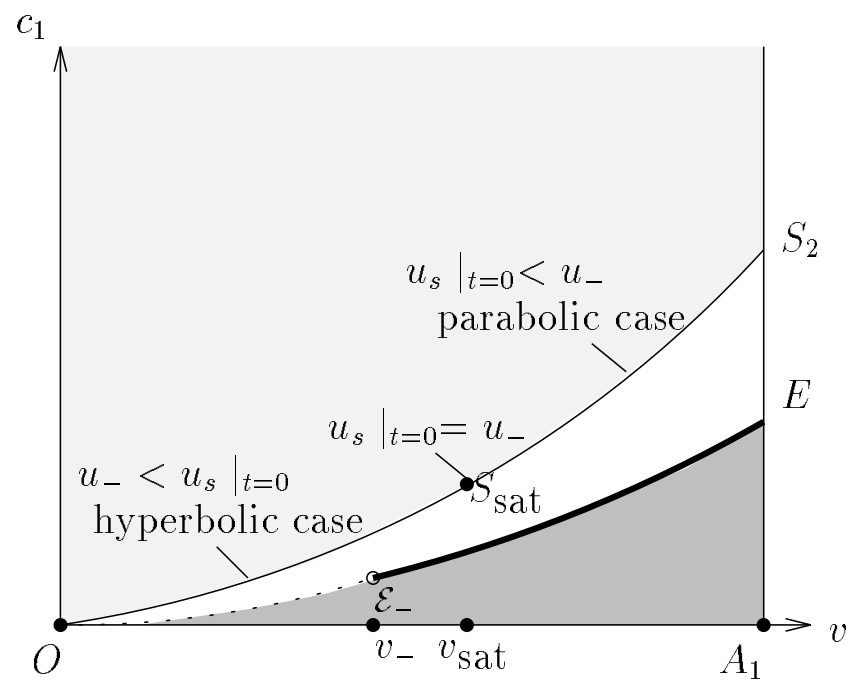

Figure 17 (ii) : $\alpha>1$

non-accessible points:

and on $\mathcal{E}$, with $\mathcal{E}_{-}$accessible

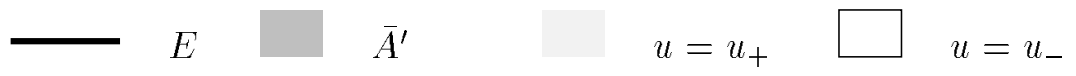

Figure 17

with $u=u_{+}$along $\gamma_{+}$. So, since $(\alpha-1) \beta>0$ (from section 1.1 and since $\alpha>1), v>0, c_{2} \geq 0, c_{1}>0, u_{+}>0$ (from section 1.1 ),$h(v)>0$ (from $(1.7)$ in section 1.1.3) and $p_{2}>0$ (from section 6.1.2), it comes $\dot{\psi}>0$ along $\gamma_{+}$: this is contradictory with $\psi\left(t_{1}\right) \geq 0$ and $\psi\left(t_{2}\right)<0$ knowing that $t_{1}<t_{2}$. 
THEOREM 6.6. Let us assume that the positive integers $n_{1}, n_{2}$ do not satisfy $\left(n_{2}=1\right.$ and $\left.n_{1} \geq 2\right)$.

Each optimal control law has at most two switchings and each optimal trajectory is of the form $\gamma_{+} \gamma_{-} \gamma_{s}$, where each arc of this sequence may be empty. So we have the following result:

- For optimal trajectories arriving near $S_{2}$, the synthesis can be topologically described by an invariant foliation $\left(v=v_{0}\right)$. Let $v_{\text {sat }}$ denote the $v$-coordinate of the saturation point $S_{\text {sat }}$; the syntheses in each leaf $\left(v=v_{0}\right)$ are shown by Figure 8 in section 1.4.3:

if $v_{0}<v_{\text {sat }}$, optimal trajectories are of the form $\gamma_{+} \gamma_{-} \gamma_{s}$ (see Figure 8 (i)) if $v_{0} \geq v_{\text {sat }}$, optimal trajectories are of the form $\gamma_{+} \gamma_{-}$(see Figure 8 (ii)) where each arc of these sequences may be empty.

- For optimal trajectories arriving near $\mathcal{E}$, the situation is intricated. Let $v_{-}$ denote the v-coordinate of $\mathcal{E}_{-}$defined in Lemma 6.5. Near a point $\mathcal{E}_{0} \neq \mathcal{E}_{-}$ of $\mathcal{E}$, the optimal synthesis is described by a $\mathcal{C}^{0}$ invariant foliation $\mathcal{F}: v=v_{0}$ the leaves of which are given by Figures 9 (i) and (ii); near $\mathcal{E}_{-}$there is no such foliation and the synthesis is given by Figure 9 (iii).

To prove this theorem, we have to set preliminary lemmas, which hold for any positive integers $n_{1}, n_{2}$.

Lemma 6.7. To prove Theorem 6.6, it is sufficient to show that no sequence $\gamma_{s} \gamma_{-}\left(\right.$with $\gamma_{s}$ and $\gamma_{-}$non-empty) can appear in a BC-extremal trajectory.

Proof. Consider sections 6.3.2 and 6.4.1.

LEMMA 6.8. Let $\gamma_{s} \gamma_{-}$(with $\gamma_{s}$ and $\gamma_{-}$non-empty) be a sequence appearing in a BC-extremal trajectory according to Figure 18

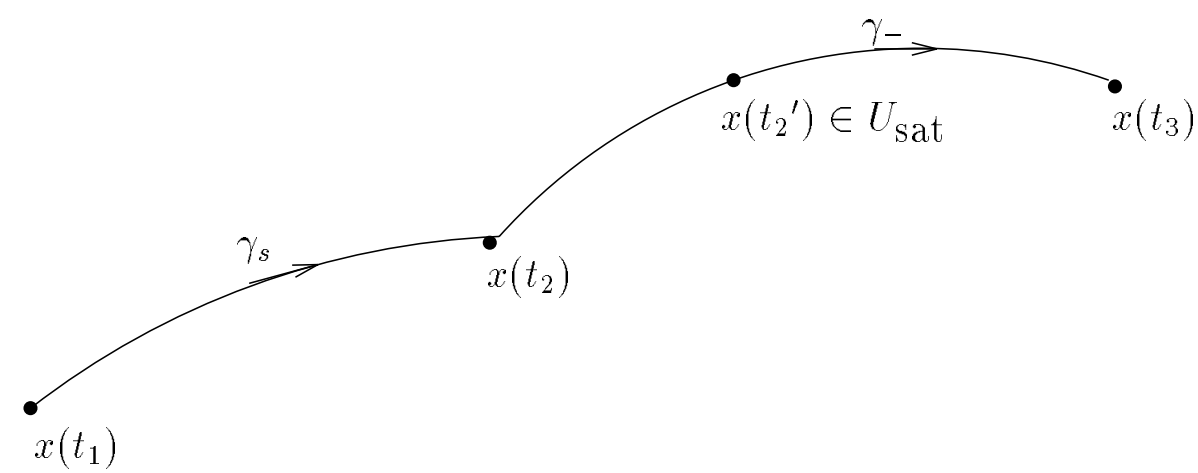

Figure 18

where $t_{2}$ and $t_{3}$ are switching times.

Consider the saturating set $\left(u_{s}=u_{-}\right)$denoted by $U_{\text {sat }}$. Then, on the considered arc $\gamma_{-}, \lim _{t \rightarrow t_{2}^{+}} \dot{\psi}(t) \leq 0$ and there exists $\left.t_{2}^{\prime} \in\right] t_{2}, t_{3}\left[\right.$ such that $\dot{\psi}\left(t_{2}^{\prime}\right)=0$, i.e. $x\left(t_{2}^{\prime}\right) \in U_{\text {sat }}$.

Proof. From (6.10) in section 6.4.1, we know that along $\gamma_{-}$, at any time in ]$t_{2}, t_{3}[$

$$
\dot{\psi}=(\alpha-1) \beta c_{2}^{n_{2}-1} v^{\alpha-2}\left[n_{2} c_{1}^{n_{1}} v^{2}+\alpha u_{-} c_{2} h(v)\right] p_{2} .
$$


Moreover, since $x\left(t_{2}\right) \in \gamma_{s}, u_{s}\left(t_{2}\right)$ is admissible and thus $u_{s}\left(t_{2}\right) \geq u_{-}$, that is $-\frac{n_{2}}{\alpha} \frac{c_{1}^{n_{1}} v^{2}}{c_{2} h(v)} \geq u_{-}$(cf. (6.7) in section 6.2.1). Hence we get $\lim _{t \rightarrow t_{2}^{+}} \dot{\psi}(t) \leq 0$ since $\beta>0, c_{2} \geq 0, v>0$ from section $1.1, p_{2}>0$ from section 6.1 .2 and since we assume $\alpha>1$.

Since $x\left(t_{2}\right) \in \gamma_{s}, \Phi\left(t_{2}\right)=\dot{\Phi}\left(t_{2}\right)=0$; and $\Phi\left(t_{3}\right)=0$ since $t_{3}$ is a switching time.

Then, $\Phi$ being $\mathcal{C}^{1}$ on the considered $B C$-extremal, Rolle's Theorem yields the existence of $t^{\prime}$ in $] t_{2}, t_{3}\left[\right.$ such that $\dot{\Phi}\left(t^{\prime}\right)=0$ since $\Phi\left(t_{2}\right)=\Phi\left(t_{3}\right)=0$. So we get from $\psi=\dot{\Phi} / h(v)$ (cf. proof of section 6.4.1) that $\psi\left(t_{2}\right)=\psi\left(t^{\prime}\right)=0$.

Then, $\psi$ being continuous on the considered $B C$-extremal and differentiable except at switching points, Rolle's Theorem yields the existence of $t_{2}^{\prime}$ in $] t_{2}, t^{\prime}[\subset] t_{2}, t_{3}\left[\right.$ such that $\dot{\psi}\left(t_{2}^{\prime}\right)=0$.

Finally we get the result, since it comes from (6.10) in section 6.4.1 and (6.7) in section 6.2.1 that:

$$
\text { along } \gamma_{-}\left[\text {where } u=u_{-}\right] \dot{\psi}=\alpha(\alpha-1) \beta c_{2}^{n_{2}} v^{\alpha-2}\left(u_{-}-u_{s}\right) h(v)
$$

and since $\beta>0, c_{2}>0, v>0$ from section 1.1 (indeed the initial time is lower than $\left.t_{2}\right), h(v)>0(\mathrm{cf}$. (1.7) in section 1.1.3) and since we assume $\alpha>1$.

Lemma 6.9. Any singular arc of a BC-extremal trajectory satisfies $\dot{c}_{2}>0$.

Proof. Along any singular extremal $\dot{\Phi} \equiv 0$ (cf. section 2.1 .4 and $(2.3)$ in section 2.1.2) that is, from the value of $\dot{\Phi}$ (cf. (6.6) in section 6.1.4) and from (1.7) in section 1.1.3:

$$
p_{1}=\left(c_{1}^{n_{1}}-\alpha \beta v^{\alpha-1} c_{2}^{n_{2}}\right) p_{2}
$$

Then

- at any negative time, since $p_{1}>0$ and $p_{2}>0$ (cf. section 6.1.2), it comes $c_{1}^{n_{1}}-\alpha \beta v^{\alpha-1} c_{2}^{n_{2}}>0$, which implies in the considered case $\alpha>1$, $\dot{c}_{2}=v\left(c_{1}^{n_{1}}-\beta v^{\alpha-1} c_{2}^{n_{2}}\right)>0$ (indeed $c_{2} \geq 0$ and $v>0$ from (1.6) in section $1.1 .3)$

- at time 0 (eventually), (6.12) becomes $c_{1}^{n_{1}}(0)=\alpha \beta v^{\alpha-1}(0) d_{2}^{n_{2}}$ (cf. (6.3) in section 6.1.2) which implies in the considered case $\alpha>1, \dot{c}_{2}(0)=$ $v(0)\left(c_{1}^{n_{1}}(0)-\beta v^{\alpha-1}(0) d_{2}^{n_{2}}\right)>0$ (indeed $d_{2}>0$ and $v(0)>0$ from section $1.1)$.

Lemma 6.10. Consider the adapted coordinate:

$$
y=c_{2} / c_{1}^{n_{1}}
$$

introduced because of the saturating set $U_{\text {sat }}$ defined in the previous Lemma 6.8, the equation $\left(u_{s}=u_{-}\right)$of which becomes:

$$
y=n_{2} \sigma(v) \text { with } \sigma(v)=-\frac{v^{2}}{\alpha u_{-} h(v)}
$$

Then

$$
\dot{y}=\frac{\dot{c}_{2}}{c_{1}^{n_{1}}}+n_{1} \frac{c_{2}}{c_{1}} v
$$


Proof. Equation (6.14) comes from the value of $u_{s}$ (cf. (6.7) in section 6.2.1) and equation (6.15) comes from (6.13) and from system (1.5) in section 1.1.2.

Proof of Theorem 6.6. Let us assume that the positive integers $n_{1}, n_{2}$ do not satisfy $\left(n_{2}=1\right.$ and $\left.n_{1} \geq 2\right)$.

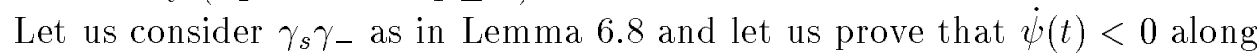
$\gamma_{-}$at any time $\left.\left.t \in\right] t_{2}, t_{3}\right]$, which is contradictory with $\dot{\psi}\left(t_{2}^{\prime}\right)=0$ : thus we prove that such a sequence $\gamma_{s} \gamma_{-}$cannot exist, and from Lemma 6.7 the theorem is proved.

Indeed $\gamma_{s}$ satisfies $\dot{c}_{2}>0$ (cf. Lemma 6.9 ) and so $\dot{c}_{2}\left(t_{2}\right)>0$, which implies $\dot{y}\left(t_{2}\right)>0$ (cf. (6.15) in Lemma 6.10, with $c_{1}>0, c_{2} \geq 0, v>0$ from (1.6) in section 1.1.3). Let us then prove that $\dot{y} \geq 0$ along $\gamma_{-}$by proving that the existence of $\left.\tau \in] t_{2}, t_{3}\right]$ with $\dot{y}(\tau)<0$ is impossible.

Indeed, if such a $\tau$ exists, there exists $\left.\tau^{\prime} \in\right] t_{2}, \tau\left[\right.$ such that $\dot{y}\left(\tau^{\prime}\right)=0$ and $\dot{y}<0$ on $\left.] \tau^{\prime}, \tau\right]$ since $\dot{y}$ is a continuous function of time with $\dot{y}\left(t_{2}\right)>0$ and $\dot{y}(\tau)<0$; and this is impossible since $\dot{y}\left(\tau^{\prime}\right)=0$ with $\left.\tau^{\prime} \in\right] t_{2}, t_{3}[$ implies that $y^{(2)}\left(\tau^{\prime}\right)>0$ [and so at any time $t>\tau^{\prime}$ close enough to $\tau^{\prime}, \dot{y}\left(\tau^{\prime}\right) \geq 0$ ]: indeed, from (6.13) and (6.15) in Lemma 6.10 and from (1.5) in section 1.1 .2

$$
\dot{y}=v\left[1-\beta y^{n_{2}} c_{1}^{n_{1}\left(n_{2}-1\right)} v^{\alpha-1}+n_{1} y c_{1}^{n_{1}-1}\right]
$$

and so, at time $\left.\tau^{\prime} \in\right] t_{2}, t_{3}\left[\right.$ such that $\dot{y}\left(\tau^{\prime}\right)=0$, computations yield

$$
\begin{aligned}
& y^{(2)}\left(\tau^{\prime}\right)=n_{1}\left(n_{1}\left(n_{2}-1\right)-\left(n_{1}-1\right)\right) c_{1}^{n_{1}-2}\left(\tau^{\prime}\right) c_{2}\left(\tau^{\prime}\right) v^{2}\left(\tau^{\prime}\right) \\
& +n_{1}\left(n_{2}-1\right) c_{1}^{n_{1}-1}\left(\tau^{\prime}\right) v^{2}\left(\tau^{\prime}\right)-(\alpha-1) \beta \frac{c_{2}^{n_{2}}\left(\tau^{\prime}\right)}{c_{1}^{n_{1}}\left(\tau^{\prime}\right)} v^{\alpha-1}\left(\tau^{\prime}\right) h\left(v\left(\tau^{\prime}\right)\right) u_{-}
\end{aligned}
$$

and so $y^{(2)}\left(\tau^{\prime}\right)>0$ since $\beta>0, u_{-}<0, c_{1}\left(\tau^{\prime}\right)>0, c_{2}\left(\tau^{\prime}\right)>0, v\left(\tau^{\prime}\right)>0$ from section 1.1 (indeed the initial time is lower than $\left.t_{2}\right), h\left(v\left(\tau^{\prime}\right)\right)>0$ (cf. (1.7) in section 1.1.3) and since $n_{1}\left(n_{2}-1\right)-\left(n_{1}-1\right) \geq 0$ under the assumption that the positive integers $n_{1}, n_{2}$ do not satisfy $n_{2}=1$ and $n_{1} \geq 2$.

Then:

- since we know from $(6.11)$ that along $\gamma_{-}, \operatorname{sign}(\dot{\psi})=\operatorname{sign}\left(u_{-}-u_{s}\right)$ with:

$$
u_{-}-u_{s}=u_{-}+\frac{n_{2} E_{1}}{\alpha R} \frac{v}{y \ln ^{2}\left(v / A_{1}\right)}
$$

from (6.7) in section 6.2.1.

- since we just proved that $\dot{y} \geq 0$ along $\gamma_{-}$, and since

$$
\frac{d}{d t}\left(\frac{v}{\ln ^{2}\left(v / A_{1}\right)}\right)=\frac{\left(\ln \left(v / A_{1}\right)-2\right) h(v) u_{-}}{\ln ^{3}\left(v / A_{1}\right)}<0
$$

with $v \in] 0, A_{1}\left[\right.$ and $y>0$, so that along $\gamma_{-}, u_{-}-u_{s}$ is a decreasing function of time,

- since from Lemma $6.8 \lim _{t \rightarrow t_{2}^{+}} \dot{\psi}(t) \leq 0$ it finally turns out that at any time $\left.t \in] t_{2}, t_{3}\right] \dot{\psi}(t)<0$ (see Figure 19 ). 


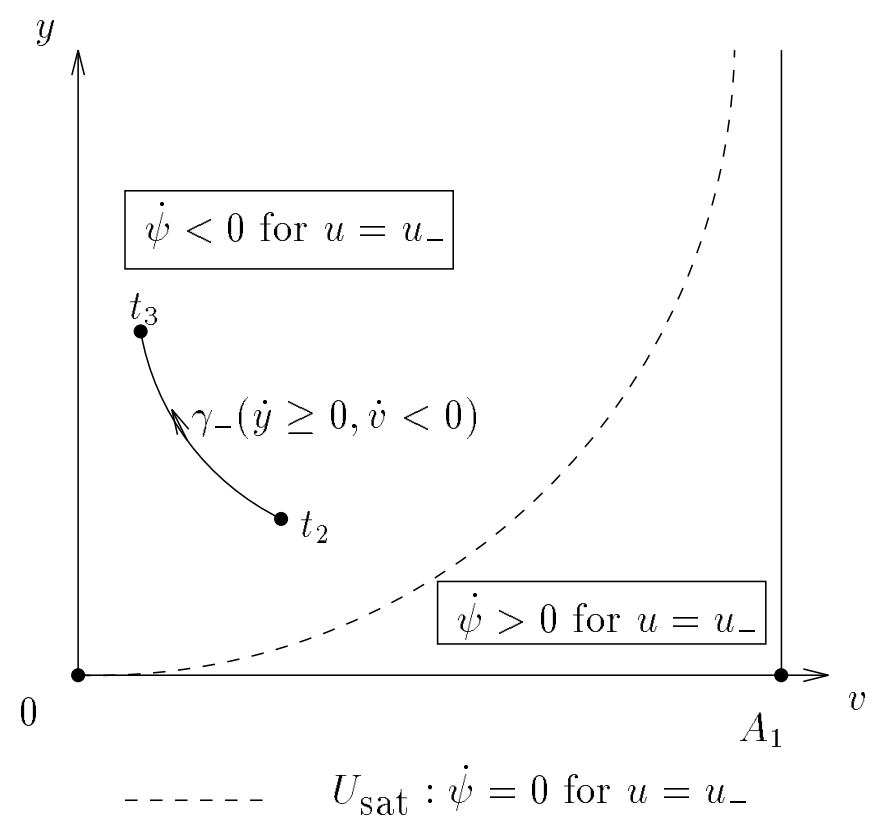

Figure 19

\section{Conjugate and FOCAL POINT ALONG A Singular EXTREmal}

\subsection{Preliminaries}

In this section we present the results of [3], [4] concerning the concept and the computation of conjugate and focal point. Our aim is to apply this theory to chemical batch reactors, hence for simplicity we restrict our study to systems of the form

$$
\dot{v}=X(v)+u Y(v) \quad, \quad u(t) \in \mathbb{R}
$$

where $X, Y$ are analytic vector fields in $\mathbb{R}^{3}$ and where $v=(x, y, z)$, but the results can be easily extended to single-input systems in $\mathbb{R}^{n}$. The class of admissible controls is the set $\mathcal{U}$ defined in section 2.1.1. Let $\gamma$ be a reference singular extremal defined on $\left[0, t_{F}\right]$; we assume that $\gamma$ satisfies the assumptions $\left(H_{0}^{\ell}\right)-\left(H_{3}^{\ell}\right)$ defined in section 2.2.2. Moreover we suppose that $\gamma$ is hyperbolic. Using the notations $D=-\operatorname{det}\left(Y,[X, Y], \operatorname{ad}^{2} Y(X)\right)$, $D^{\prime}=\operatorname{det}\left(Y,[X, Y], \operatorname{ad}^{2} X(Y)\right)$ and $D^{\prime \prime}=\operatorname{det}(Y,[X, Y], X)$ introduced in section $2.4, \gamma$ is solution to the differential equation in $\mathbb{R}^{3}$

$$
\dot{v}=S(v)
$$

where $S(v)=X(v)+u_{s}(v) Y(v), u_{s}(v)=-D^{\prime}(v) / D(v)$ is the singular control feedback and $\gamma$ is contained in the set $D^{\prime \prime}(v) D(v)>0$.

According to theorem 2.5 we denote by $t_{1 c}$ the first conjugate time along $\gamma$, that is $\gamma\left(t_{1 c}\right)$ is the first point where $\gamma$ ceases to be a time-minimizer in the $\mathcal{C}^{0}$ topology with respect to the set of curves having the same initial and final points. In [3] we describe an algorithm to compute such a point. We can generalize this algorithm to deal with general end-points problems and this leads to the concept of focal point.

ESAIM: CoCv, DECEMBER 1998, VoL. 3, 407-467 


\subsection{First method of COMPUting CONJUgate POINTS}

We briefly recall the method of [3], [4] to compute the conjugate points in the hyperbolic situation

7.2.1. Normal Form. Using adapted coordinates and a feedback, the system (7.1) can be written

$$
\left\{\begin{array}{l}
\dot{x}=1+Q_{1}(x, y, z) \\
\dot{y}=z+Q_{2}(x, y, z) \\
\dot{z}=u
\end{array}\right.
$$

where the $Q_{i}$ 's are power series of the variable $(y, z)$ with coefficients in $\mathbb{R}[x]$ and containing monomials of degree $\geq 2$, the reference trajectory $\gamma$ being identified to $t \mapsto(t, 0,0)$ and corresponding to the zero control. We write $Q_{1}(x, y, z)=a(x) z^{2}+2 b(x) y z+c(x) y^{2}+R$ where the remaining term $R$ is of the form $o(y, z)^{2}$, and we introduce $L(t, y, z)=a(t) z^{2}+2 b(t) y z+c(t) y^{2}$.

By assumption $\gamma$ is hyperbolic and hence $a(t)<0$ for $t \in\left[0, t_{F}\right]$. From [4], to characterize the optimality of the reference trajectory $\gamma$ we can restrict our study to the model:

$$
\left\{\begin{array}{l}
\dot{x}=1+L(t, y, z) \\
\dot{y}=z \\
\dot{z}=u
\end{array}\right.
$$

7.2.2. BASIC FACT. By definition $\gamma: t \mapsto(t, 0,0)$ is time optimal on $\left[0, t_{F}\right]$ if for any $t \in\left[0, t_{F}\right]$ the point $(t, 0,0)$ is not accessible from $(0,0,0)$ in a time $t^{\prime}<t$.

Hence, let $v(t)=(t, 0,0)+\varphi(t)$ with $\varphi=\left(\varphi_{1}, \varphi_{2}, \varphi_{3}\right)$ be a solution to the model (7.4), then $\varphi(t)$ is solution to

$$
\dot{\varphi}_{1}=L\left(t, \varphi_{2}, \varphi_{3}\right), \dot{\varphi_{2}}=\varphi_{3}, \dot{\varphi_{3}}=u
$$

Observe that the condition $v\left(t^{\prime}\right)=(t, 0,0)$ implies $\varphi_{2}\left(t^{\prime}\right)=\varphi_{3}\left(t^{\prime}\right)=0$ and hence $\gamma$ is a time minimizer on $\left[0, t_{F}\right]$ if and only if the functional

$$
J(t)=\int_{0}^{t}\left(a(s) \varphi_{3}^{2}+2 b(s) \varphi_{2} \varphi_{3}+c(s) \varphi_{2}^{2}\right) d s
$$

satisfies $J(t) \leq 0$ for any $t \in\left[0, t_{F}\right]$ when evaluated on the set of curves $\varphi_{2}, \varphi_{3}$ solutions to the equations: $\dot{\varphi}_{2}=\varphi_{3}, \dot{\varphi}_{3}=u$ with the boundary conditions: $\varphi_{2}(0)=\varphi_{3}(0)=\varphi_{2}\left(t_{F}\right)=\varphi_{3}\left(t_{F}\right)=0$.

Now, since $u \in \mathbb{R}$, the variable $\varphi_{3}$ can be taken as the control (this corresponds to the concept of reduced system defined in section 3.2) and we have to study the sign of $J$ on the set $\mathcal{C}$ of non-zero smooth curves $\varphi_{2}$ with $\dot{\varphi}_{2}=\varphi_{3}$ (control) and satisfying the boundary conditions: $\varphi_{2}(0)=\varphi_{2}\left(t_{F}\right)=$ 0 (the constraints on $\varphi_{3}$ have been relaxed).

7.2.3. Definition. Let $t_{1 c}$ be the first time $\left.\left.t \in\right] 0, t_{F}\right]$ such that the maximum of $J(t)$ on $\mathcal{C}$ is zero.

According to classical calculus of variations we have: 
LEMMA 7.1. If $t<t_{1 c}$ then $J(t)<0$ on $\mathcal{C}$ and if $t>t_{1 c}$ the maximum of $J(t)$ on $\mathcal{C}$ is $+\infty$. In particular if $t<t_{1 c}$ then $\gamma$ is a time minimizer on $\left[0, t_{F}\right]$ and if $t>t_{1 c} \gamma$ is no more a minimizer. Hence $\gamma\left(t_{1 c}\right)$ is the first conjugate point to $\gamma(0)$. The time $t_{1 c}$ is the first time $t>0$ such that there exists a non-trivial solution $\varphi_{2}$ to Euler-Lagrange equation:

$$
\frac{d}{d s} \frac{\partial L}{\partial \dot{\varphi}_{2}}-\frac{\partial L}{\partial \varphi_{2}}=0
$$

with $\varphi_{2}(0)=\varphi_{2}\left(t_{F}\right)=0$ and $L\left(s, \varphi_{2}, \dot{\varphi}_{2}\right)=a(s) \dot{\varphi}_{2}^{2}+2 b(s) \varphi_{2} \dot{\varphi}_{2}+c(s) \varphi_{2}^{2}$

\subsection{Conjugate points and the synthesis problem}

7.3.1. Preliminaries. Let $0<M \leq+\infty$ and consider now the control system $\dot{v}=X+u Y$, where the set of admissible controls is the set $\mathcal{U}_{M}$ of measurable mappings taking their values in $[-M, M]$.

Recall that $S=X-\left(D^{\prime} / D\right) Y$ is the vector field whose non-periodic trajectories are singular extremals satisfying $\left(H_{0}^{\ell}\right)-\left(H_{3}^{\ell}\right)$. Let $\gamma$ be such a reference trajectory defined on $\left[0, t_{F}\right]$ and corresponding to a control taking its values in $]-M, M\left[\right.$; and let us assume that $\gamma$ is hyperbolic. Let $V(t), t \in\left[0, t_{F}\right]$ be the solution to the variational equation

$$
\delta \dot{v}(t)=\frac{\partial S}{\partial v}(\gamma(t)) \delta v(t)
$$

with initial condition $V(0)=Y(\gamma(0))$.

If $Z$ is a vector field, we denote by exp $t Z$ the local one-parameter group associated to $Z$. Let $\varepsilon, \varepsilon^{\prime}= \pm 1$ and let $g$ be the mapping:

$g:\left(t_{1}, t_{2}, t_{3}, \varepsilon, \varepsilon^{\prime}\right) \mapsto \exp t_{3}\left(X+\varepsilon^{\prime} M Y\right) . \exp t_{2} S . \exp t_{1}\left(X+\varepsilon^{\prime} M Y\right)(\gamma(0))$

From section 2.3 , since $\gamma$ is hyperbolic, such a trajectory is an extremal for $t_{1}, t_{3} \geq 0$ and small. Let $\mathcal{F}$ be the image of $g$ for $t_{2} \in\left[0, t_{F}\right]$ and $t_{1}, t_{3}$ sufficiently small.

According to [3] if $\operatorname{det}(V(\gamma(t)), Y(\gamma(t)), S(\gamma(t)))$ is never vanishing on ] $\left.0, t_{F}\right]$ then $\mathcal{F}$ is an extremal field about the arc $\gamma$ in the following sense: there exists a $\mathcal{C}^{0}$-neighborhood $U$ of $\gamma$ such that each point of $U$ is the image of an unique $\left(t_{1}, t_{2}, t_{3}, \varepsilon, \varepsilon^{\prime}\right)$. Moreover it is the time optimal synthesis in a neighborhood of the reference trajectory for the fixed end point problem.

7.3.2. Notation. Let us denote by $t_{1 c}^{\prime}$ the first time $\left.\left.t \in\right] 0, t_{F}\right]$ such that $\operatorname{det}(V(\gamma(t)), Y(\gamma(t)), S(\gamma(t)))$ vanishes. The following lemma is proved in $[3]$.

LEMMA 7.2. We have the following results:

(i) $V(t) \in \operatorname{Span}\{Y(\gamma(t)),[X, Y](\gamma(t))\}$

(ii) $\operatorname{det}(V(\gamma(t)), Y(\gamma(t)), S(\gamma(t)))=0$ for $t \in] 0, t_{F}$ if and only if $V(t)$ and $Y(\gamma(t))$ are colinear

(iii) $t_{1 c}=t_{1 c}^{\prime}$

7.3.3. Curvature. Assume that the system is in the normal form described in section 7.2.1, where $\gamma$ is identified to $t \mapsto(t, 0,0)$ and corresponds to the zero control. The variational equation along $\gamma$ takes the form:

$$
\delta \dot{x}=0, \quad \delta \dot{y}=\delta z, \quad \delta \dot{z}=\frac{c-\dot{b}}{a} \delta y-\frac{\dot{a}}{a} \delta z
$$


and the last two equations can be written as the second-order differential equation

$$
\delta \ddot{y}+\frac{\dot{a}}{a} \delta \dot{y}+\frac{\dot{b}-c}{a} \delta y=0
$$

The existence of conjugate point means that there exists a non trivial solution to (7.9) satisfying $\delta y(0)=\delta y\left(t_{1 c}\right)=0$. If we set $A=\frac{\dot{a}}{a}, B=\frac{\dot{b}-c}{a}$ and $\delta y(t)=C(t) J(t)$ where $C(t)=\exp \int_{0}^{t}-\frac{A(s)}{2} d s$ the previous equation can be written in the normal form:

$$
\ddot{Y}+K Y=0
$$

where $K=\ddot{C}+A \dot{C}+B C, C=1 / \sqrt{|a|}, K$ being defined on the set $D D^{\prime \prime}>0$ and corresponds to the concept of curvature in the hyperbolic case.

7.3.4. Geometric interpretation. First, let us assume that the system coincides with the model:

$$
\dot{x}=1+L(t, y, z), \dot{y}=z, \dot{z}=u
$$

and the associated reduced system defined in section 3.2 is then:

$$
\dot{x}=1+L(t, y, z), \dot{y}=z
$$

where $z$ is the control. By definition of $t_{1 c}$ there exists a curve $\bar{y}$ such that $\dot{\bar{y}}(0)=1, y(0)=y\left(t_{1 c}\right)=0, \bar{y}(t) \neq 0$ on $] 0, t_{1 c}\left[\right.$ and $\int_{0}^{t_{1 c}} L(t, y, \bar{y}) d t=$ 0 . Hence the corresponding solution $(\bar{x}, \bar{y})$ initiating from $(0,0)$ satisfies $x\left(t_{1 c}\right)=t_{1 c}$ and $(\bar{x}, \bar{y})$ intersects the set $\left(\left[0, t_{1 c}\right], 0\right)$ only at $\left(t_{1 c}, 0\right)$.

Let $\varepsilon \in \mathbb{R}$ and let $\bar{x}_{\varepsilon}$ be the solution starting from 0 and corresponding to the control $z=\varepsilon \bar{y}$. Our analysis shows that the family of curves $\left(\bar{x}_{\varepsilon}, \varepsilon \bar{y}\right)$ intersects (] $\left.\left.0, t_{1 c}\right], 0\right)$ only at $\left(t_{1 c}, 0\right)$.

Consider now the system (7.1) written in the normal form (7.3) where $Y$ is identified to $\frac{\partial}{\partial z}$ and $\gamma$ to $t \mapsto(t, 0,0)$. Let $\Pi$ be the projection $(x, y, z) \mapsto(x, y)$. Let $\varepsilon \in \mathbb{R}$ and let $\left(\tilde{x}_{\varepsilon}, \tilde{y}_{\varepsilon}\right)$ be the solution initiating from 0 and corresponding to the control $z=\varepsilon \bar{y}$. Our study shows that the point $\left(t_{1 c}, 0\right)=\Pi(\gamma)\left(t_{1 c}\right)$ is the limit point of the intersections of curves $\left(\tilde{x}_{\varepsilon}, \tilde{y}_{\varepsilon}\right)$ with the axis $y=0$. This corresponds to the geometric interpretation of conjugate points in classical calculus of variations : $\Pi(\gamma)\left(t_{1 c}\right)$ is the limit point of the intersections of the extremals in a neighborhood of the reference extremal for the reduced system.

\subsection{FOCAL POINTS}

We can extend the previous concept of conjugate point to deal with optimal problems where the end point belongs to a manifold. For simplicity we restrict our study to a terminal manifold of codimension one. 
7.4.1. Normal Form. We consider system $(7.1) \dot{v}=X(v)+u Y(v)$ and we suppose now that the admissible controls are the measurable mappings satisfying $|u| \leq 1$. We assume that the terminal manifold is a regular submanifold of codimension one and moreover we suppose that $Y$ is tangent everywhere to $N$ (flat case). Let $\gamma$ be an extremal satisfying the assumptions $\left(H_{0}^{\ell}\right)-\left(H_{3}^{\ell}\right)$; and let us assume that $\gamma$ is hyperbolic and that the singular control $u_{s}$ corresponding to $\gamma$ is such that $\left.\left.u_{s}\right|_{\gamma} \in\right]-1,+1[$. We identify the terminal point $\gamma(0)$ to 0 and we assume that $\gamma$ is a BC-extremal, that is it satisfies the transversality condition which takes the form: the tangent space to $N$ at 0 is spanned by $Y(0),[X, Y](0)$. Moreover we assume that the set of points where $[\mathrm{X}, \mathrm{Y}]$ is tangent to $N$ is near 0 the image of a simple curve $\delta: \varepsilon \mapsto \mathbb{R}^{3}, \delta(0)=0$, transverse to $Y$. Straightforward computations show that the system can be written in a tubular neighborhood of $\gamma$ in adapted coordinates in the following normal form:

$$
\left\{\begin{array}{l}
\dot{x}=1+a(x) z^{2}+2 b(x) y z+c(x) y^{2}+R_{1} \\
\dot{y}=d(x)+e(0) z+R_{2} \\
\dot{z}=\left(u-\left.u_{s}\right|_{\gamma}(x)\right)+f(x) y+g(0) z+R_{3}
\end{array}\right.
$$

where $b(0)=0, e(0) \neq 0$ and $R_{1}$ (resp. $R_{2}, R_{3}$ ) are remaining terms of order $\geq 3$ (resp. $\geq 2$ ) in $(y, z)$. The reference extremal $\gamma$ is identified to $t \mapsto(t, 0,0)$, the target $N$ to $x=0$ and the image of $\delta$ to the axis $0 y$. Since $\gamma$ is hyperbolic we have $a(x)<0$ for $x \in\left[t_{0}, 0\right]$.

7.4.2. Optimal synthesis and fOcal points. From the classification of $[5], \gamma(0)=0$ is an hyperbolic point and the optimal synthesis for the time minimal problem with terminal manifold $N$ can be easily described near 0 . We shall extend this synthesis to a tubular neighborhood of $\gamma$. For $t \leq 0$, let $\Sigma_{t}$ be the set of BC-singular extremals ending on $\operatorname{Im} \delta$ in a time $|s|$, $0 \geq s \geq t$. It can be written as $\Sigma_{t}=\bigcup_{0 \geq s \geq t}(\exp s S)(\operatorname{Im} \delta)$ where $S$ is the vector field defined in section 7.1 whose solutions are the singular extremals. For $t$ small enough, $\Sigma_{t}$ is a smooth surface. By assumption $W=\delta^{\prime}(0)$ belongs to $\operatorname{Span}\{Y(0),[X, Y](0)\}$. Let $\lambda_{1}, \lambda_{2}$ be two scalars such that $W=\lambda_{1} Y(0)+\lambda_{2}[X, Y](0)$ and let $Z$ be the vector field $\lambda_{1} Y+\lambda_{2}[X, Y]$. Let $t$ be fixed, the derivative at 0 of the curve $\varepsilon \mapsto \delta_{t}(\varepsilon)=(\operatorname{exptS})(\delta(\varepsilon))$ is denoted $W(t)$ and is given by:

$$
W(t)=\left.\frac{d}{d \varepsilon}\right|_{\varepsilon=0}(\exp t S . \exp \varepsilon Z(0))
$$

For $t \in\left[t_{0}, 0\right], \gamma(t)=\exp t S(0)$ and we can write $\exp t S . \exp \varepsilon Z(0)=$ $\exp t S . \exp \varepsilon Z$. exp $-t S(\gamma(t))$ and computing the derivative at $\varepsilon=0$, we get

$$
W(t)=\sum_{k \geq 0}(-1)^{k} \frac{t^{k}}{k !} a d^{k} S(Z)(\gamma(t))
$$

Since $S$ is the singular vector field and $Y,[X, Y]$ are independant along $\gamma$ we have:

$$
\left.\left.a d^{k} S(Y)\right|_{\gamma} \in \operatorname{Span}\{Y,[X, Y]\}\right|_{\gamma} \text { for } k \geq 0
$$

We have the following lemma

ESAIM: CoCV, DeCEMBER 1998, Vol. 3, 407-467 
LEMMA 7.3. The vector $W(t)$ is solution to the variational equation:

$$
\delta \dot{v}(t)=\frac{\partial S}{\partial v}(\gamma(t)) \delta v
$$

with initial condition $W(0)=\lambda_{1} Y(0)+\lambda_{2}[X, Y](0)$ tangent vector to $\delta$ at $\gamma(0)=0$. It belongs to $\operatorname{Span}\{Y(\gamma(t)),[X, Y](\gamma(t))\}$

7.4.3. Definition. Let $t_{1 f}$ be the first time $t \in\left[t_{0}, 0[\right.$ such that

$$
\operatorname{det}(W(t) Y(\gamma(t)), S(\gamma(t)))=0
$$

Then $\gamma\left(t_{1 f}\right)$ is called the first focal point along $\gamma$.

As for the fixed end point problem we have the following result:

Proposition 7.4. Let $0>t>t_{1 f}$, then $\Sigma_{t}$ is along $\left.\gamma\right|_{[0, t]}$ a smooth surface separating $\mathbb{R}^{3}$ into two domains. On a tubular neighborhood of $\gamma$, the optimal synthesis using the normal coordinates of section 7.4 .1 is the following: on the surface $\Sigma_{t}$ we apply the singular control, otherwise in the domain containing $x=y=0, z<0$ (resp. $z>0$ ) the optimal control is $u=+1$ (resp. $u=-1$ )

7.4.4. Geometric interpretation. Assume $Y$ identified to $\frac{\partial}{\partial z}$ and let $\Pi$ be the projection $(x, y, z) \mapsto(x, y)$. At $t=t_{1 c}$ the vector $W(t)$ becomes colinear to $Y(\gamma(t))$ and hence the surface $\Pi\left(\Sigma_{t}\right)$ has a singular point. The point $\Pi\left(\gamma\left(t_{1 c}\right)\right)$ is the limit point of the intersections of the singular extremals of the reduced system with the reference extremal. This corresponds to concept of focal point in classical calculus of variations.

As in [4] we can prove, using the normal form, the following result:

Proposition 7.5. At $t=t_{1 f}$ the reference trajectory $\Pi(\gamma)$ of the reduced system ceases to be optimal.

7.4.5. Algorithms. We have the following algorithms to compute conjugate and focal points.

- conjugate point At $t=t_{c}, V(T)$ becomes colinear to $Y(\gamma(t))$

- focal point At $t=t_{f}, W(T)$ becomes colinear to $Y(\gamma(t))$

7.4.6. Example FOR Batch ReACTORS. Consider problem $\dot{\mathcal{P}}_{1}$ when $n_{1}=$ $n_{2}=1$. The analysis of section 5 shows that $\Pi\left(\Sigma_{t}\right)$ is a smooth surface along a reference singular extremal for each time $t$ and the problem is without focal point. This explains the simplicity of the closed loop optimal control. On the contrary, numerical computations given in [3] show the existence of conjugate points in a domain of the state space.

\section{Numerical Simulations}

\subsection{Problem $\mathcal{P}_{2}$}

According to section 1.1 problem $\mathcal{P}_{2}$ is the time-minimal problem of reaching the target $\left(c_{2}=d_{2}\right)$ from an initial value $\left(c_{2}\right)_{0} \in\left[0, d_{2}\right.$ [ for the system (1.4) where the control is $v=A_{1} \exp \left(-E_{1} / R T\right)$ with $T_{m} \leq T \leq T_{M}$ and so

$$
v_{m}=A_{1} \exp \left(-E_{1} / R T_{m}\right) \leq v \leq \underset{\text { ESAIM: Cocv, December } 1998, \text { Vol. } 3,407-467}{v_{M}=A_{1} \exp \left(-E_{1} / R T_{M}\right)}
$$


From section 3.2 we know that $\mathcal{P}_{2}$ is equivalent to the projected problem $\mathcal{P}_{2}^{\prime}$ obtained by restricting our study to the planar system

$$
\left\{\begin{array}{l}
\dot{c}_{1}=-v c_{1}^{n_{1}} \\
\dot{c}_{2}=v c_{1}^{n_{1}}-\beta v^{\alpha} c_{2}^{n_{2}}
\end{array}\right.
$$

Then the adjoint vector $p=\left(p_{1}, p_{2}\right)$ associated to the state vector $x=$ $\left(c_{1}, c_{2}\right)$ satisfies

$$
\left\{\begin{array}{l}
\dot{p}_{1}=-n_{1} v c_{1}^{n_{1}-1}\left(p_{1}-p_{2}\right) \\
\dot{p}_{2}=n_{2} \beta v^{\alpha} c_{2}^{n_{2}-1} p_{2}
\end{array}\right.
$$

with at final time 0

$$
p(0)=(0,1)
$$

the corresponding Hamiltonian being:

$$
H(x, p, v)=-v c_{1}^{n_{1}} p_{1}+\left(v c_{1}^{n_{1}}-\beta v^{\alpha} c_{2}^{n_{2}}\right) p_{2}
$$

So the singular control $\hat{v}$ solution to the equation in $v: \frac{\partial H}{\partial v}(x, p, v)=0$ satisfies:

$$
\hat{v}=\left[\frac{c_{1}^{n_{1}}\left(p_{2}-p_{1}\right)}{\alpha \beta c_{2}^{n_{2}} p_{2}}\right]^{\frac{1}{\alpha-1}}
$$

As in sections 1.4 .2 and 4.3 .1 , let us consider on the target $\left(c_{2}=d_{2}\right)$ the points:

- $E_{m}=\left(\left(\beta d_{2}^{n_{2}} v_{m}^{\alpha-1}\right)^{\frac{1}{n_{1}}}, d_{2}\right)$ such that $\dot{c}_{2}=0$ when $v=v_{m}$

- $S_{m}=\left(\left(\alpha \beta d_{2}^{n_{2}} v_{m}^{\alpha-1}\right)^{\frac{1}{n_{1}}}, d_{2}\right)$ such that the final singular control is $v_{m}$

- $S_{M}=\left(\left(\alpha \beta d_{2}^{n_{2}} v_{M}^{\alpha-1}\right)^{\frac{1}{n_{1}}}, d_{2}\right)$ such that the final singular control is $v_{M}$

Giving particular values to constants and solving systems according to the joined Scilab ${ }^{1}$ file p2.s we found Figure 20, which corroborates Figure 5 in the whole physical space.

\subsection{Problem $\dot{\mathcal{P}}_{2}$}

8.2.1. Near the singular locus. According to section 1.1 problem $\dot{\mathcal{P}}_{2}$ is the time-minimal problem of reaching the target $\left(c_{2}=d_{2}\right)$ from an initial value $\left(c_{2}\right)_{0} \in\left[0, d_{2}\right.$ [ for the system (1.5) where the control is $u=\dot{T}$ with $u_{-} \leq u \leq u_{+}$and $u_{-}<0<u_{+}$From section 3.2 we know that $\dot{\mathcal{P}}_{2}$ is equivalent to the projected problem $\dot{\mathcal{P}}_{2}^{\prime}$ obtained by restricting our study to the system

$$
\left\{\begin{array}{l}
\dot{c}_{1}=-v c_{1}^{n_{1}} \\
\dot{c}_{2}=v c_{1}^{n_{1}}-\beta v^{\alpha} c_{2}^{n_{2}} \\
\dot{v}=h(v) u \text { with } h(v)=\left(R v / E_{1}\right) \ln ^{2}\left(v / A_{1}\right)
\end{array}\right.
$$

\footnotetext{
${ }^{1}$ Scilab is a package available on the Web site http://www-rocq.inria.fr/scilab ESAIM: CoCv, DeCEMBer 1998, VoL. 3, 407-467
} 


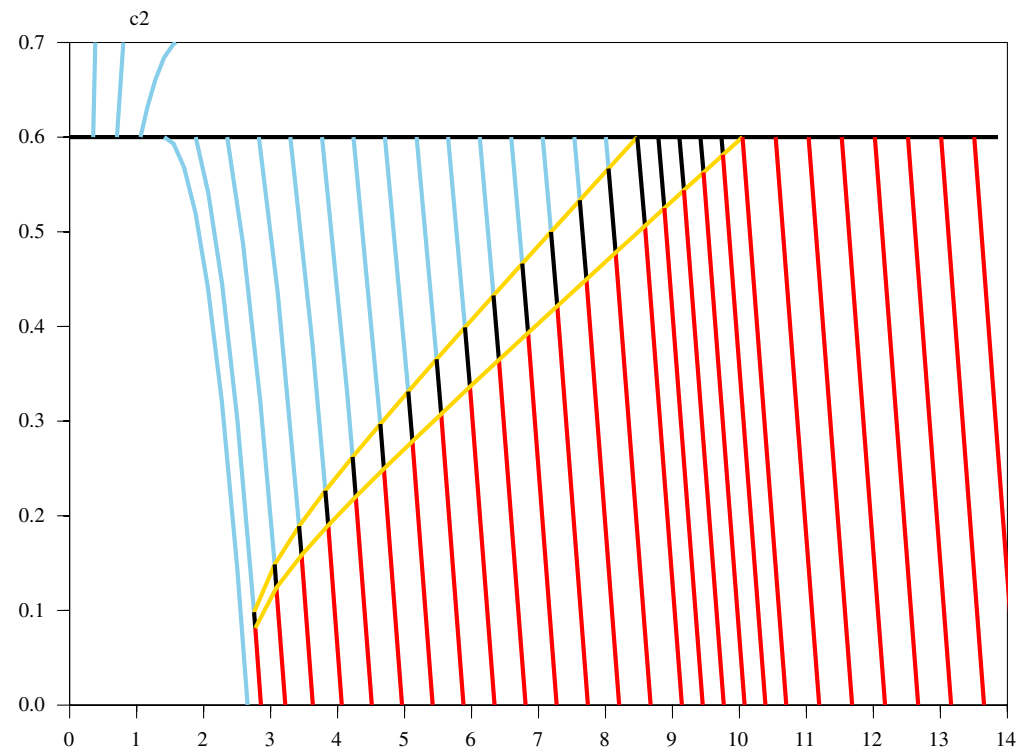

Figure 20: $\hat{\gamma}$ are black, $\gamma_{m}$ are blue, $\gamma_{M}$ are red and switching curves are yellow

Then the adjoint vector $p=\left(p_{1}, p_{2}, p_{3}\right)$ associated to the state vector $x=$ $\left(c_{1}, c_{2}, v\right)$ satisfies

$$
\left\{\begin{array}{l}
\dot{p}_{1}=n_{1} v c_{1}^{n_{1}-1}\left(p_{1}-p_{2}\right) \\
\dot{p}_{2}=n_{2} \beta v^{\alpha} c_{2}^{n_{2}-1} p_{2} \\
\dot{p}_{3}=c_{1}^{n_{1}} p_{1}+\left(-c_{1}^{n_{1}}+\alpha \beta v^{\alpha-1} c_{2}^{n_{2}}\right) p_{2}-\frac{d h}{d v}(v) u p_{3}
\end{array}\right.
$$

with at final time 0

$$
p(0)=(0,1,0)
$$

the corresponding Hamiltonian being:

$$
H(x, p, v)=-v c_{1}^{n_{1}} p_{1}+\left(v c_{1}^{n_{1}}-\beta v^{\alpha} c_{2}^{n_{2}}\right) p_{2}+h(v) u p_{3}
$$

Then, as in Lemma 6.2 in section 6.2.1, computations applying section 2.4 to the dynamical system (8.4) give the singular control $u_{s}$ :

$$
u_{s}=-\frac{n_{2}}{\alpha} \frac{c_{1}^{n_{1}} v^{2}}{c_{2} h(v)}<0
$$

As in Lemma 6.1 in section 6.2 .1 , let us consider on the target $\left(c_{2}=d_{2}\right)$ the set $S_{2}$ of points $\left(c_{1}>0, d_{2}, v \in\right] 0, A_{1}[)$ satisfying $\frac{d}{d t} \frac{\partial H}{\partial u}=0$, so that any singular $B C$-extremal arriving on $N$ arrives on $S_{2}$. Taking (8.4)-(8.7) into account, we get:

$$
S_{2}=\left\{\left(c_{1}>0, d_{2}, v \in\right] 0, A_{1}[) \in N ; c_{1}^{n_{1}}-\alpha \beta v^{\alpha-1} d_{2}^{n_{2}}=0\right\}
$$


Let us then consider (as in in Lemma 6.2 in section 6.2.1) the point $S_{\text {sat }}$ of $S_{2}$ where the final singular control is equal to $u_{-}$. From (8.8) and (8.9) it comes:

$$
S_{\mathrm{sat}}=\left(\left(\alpha \beta v_{\mathrm{sat}}^{\alpha-1} d_{2}^{n_{2}}\right)^{\frac{1}{n_{1}}}, d_{2}, v_{\mathrm{sat}}\right) \text { such that } u_{-}=-n_{2} \beta d_{2}^{n_{2}-1} \frac{v_{\mathrm{sat}}^{\alpha+1}}{h\left(v_{\mathrm{sat}}\right)}
$$

Giving particular values to constants we computed saturation points on singular trajectories and switching points on $\gamma_{-}$and $\gamma_{+}$arcs (cf. the joined Scilab file sing.s): these computations corroborate in the whole physical space the time minimal synthesis found in section 6 and shown by Figures 8 and 17(ii); in particular we found that there is no switch on $\gamma_{+}$arcs. Figure 21 (obtained when executing the joined Scilab file plsing.s after sing.s) shows the surface separating $\left(u^{*}=u_{-}\right)$from $\left(u^{*}=u_{+}\right)$, where $u^{*}$ denotes the optimal control: this surface is constituted of singular trajectories (black), of switching points for $\gamma_{+} \gamma_{-}$trajectories (blue (resp. green) when the initial point is in the parabolic (resp. hyperbolic) domain) and of first switching points for $\gamma_{+} \gamma_{-} \gamma_{s}$ trajectories (red).

Note that $\sum_{i=1}^{3} c_{i}(t)$ is constant. So, for the sake of simplicity, as we chose $n_{1}=$ $n_{2}=1$, we considered relative concentrations $c_{i}^{\prime}=c_{i} / \sum_{i=1}^{3} c_{i}(0), \mathrm{i}=1,2$. Then the state system (8.4) becomes:

$$
\left\{\begin{array}{l}
\dot{c}_{1}^{\prime}=-v c_{1}^{\prime} \\
\dot{c}_{2}^{\prime}=v c_{1}^{\prime}-\beta v^{\alpha} c_{2}^{\prime} \\
\dot{v}=h(v) u \text { with } h(v)=\left(R v / E_{1}\right) \ln ^{2}\left(v / A_{1}\right)
\end{array}\right.
$$

with $c_{1}^{\prime}+c_{2}^{\prime} \leq 1$

8.2.2. NEAR THE EXcEPTIONAL Locus. Keeping the same values, we solved dynamical and adjoint systems near the exceptional locus, defined as in Lemma 6.4 in section 6.2 .3 as the set of final points of BC-extremal trajectories tangent to the target $N:\left(c_{2}=d_{2}\right)$, included in $\mathcal{E}=\left\{\left(c_{1}>0, d_{2}, v \in\right.\right.$ ] $\left.0, A_{1}[) \in N ; c_{1}^{n_{1}}-\beta v^{\alpha-1} d_{2}^{n_{2}}=0\right\}$ the set of points of $N$ such that $\dot{c}_{2}=0$. As in Lemma 6.5 in section 6.2 we consider the point $\mathcal{E}_{-}$of $\mathcal{E}$ such that the exceptional arc arriving at this point has with $N$ a contact of order greater than two. It comes:

$$
\begin{gathered}
\mathcal{E}_{-}=\left(\left(\beta v_{-}^{\alpha-1} d_{2}^{n_{2}}\right)^{\frac{1}{n_{1}}}, d_{2}, v_{-}\right) \text {such that } \\
(1-\alpha) u_{-} \frac{R}{E_{1}} \ln ^{2}\left(\frac{v_{-}}{A_{1}}\right)=n_{1} \beta^{1-\frac{1}{n_{1}}} d_{2}^{n_{2}\left(1-\frac{1}{n_{1}}\right)} v_{-}^{\alpha+\frac{1}{n_{1}}(1-\alpha)}
\end{gathered}
$$

Then, according to the joined Scilab file exc.s we found Figure 22 (exceptional trajectories) and Figure 23 ( $\gamma$ - trajectory) which corroborate Figure 9 (iii). 


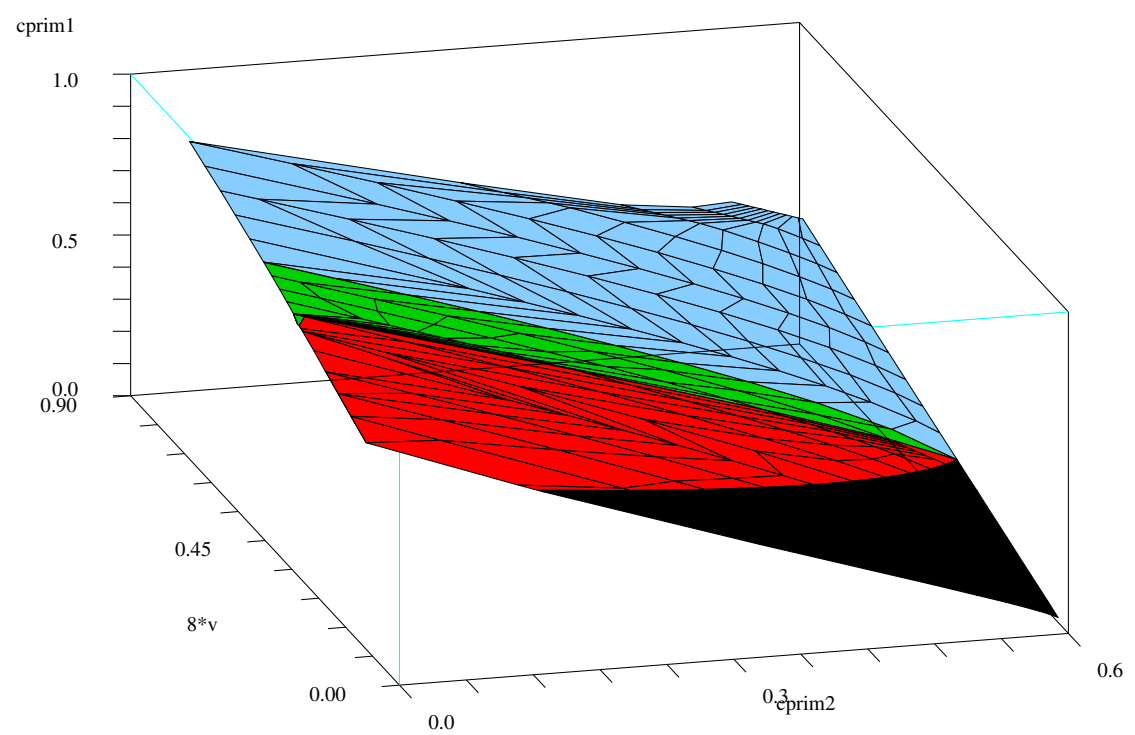

Figure 21: $\gamma_{s}$ are black, switching points for $\gamma_{+} \gamma_{-}$are blue (parabolic case) or green (hyperbolic case), first switching points for $\gamma_{+} \gamma_{-} \gamma_{s}$ are red

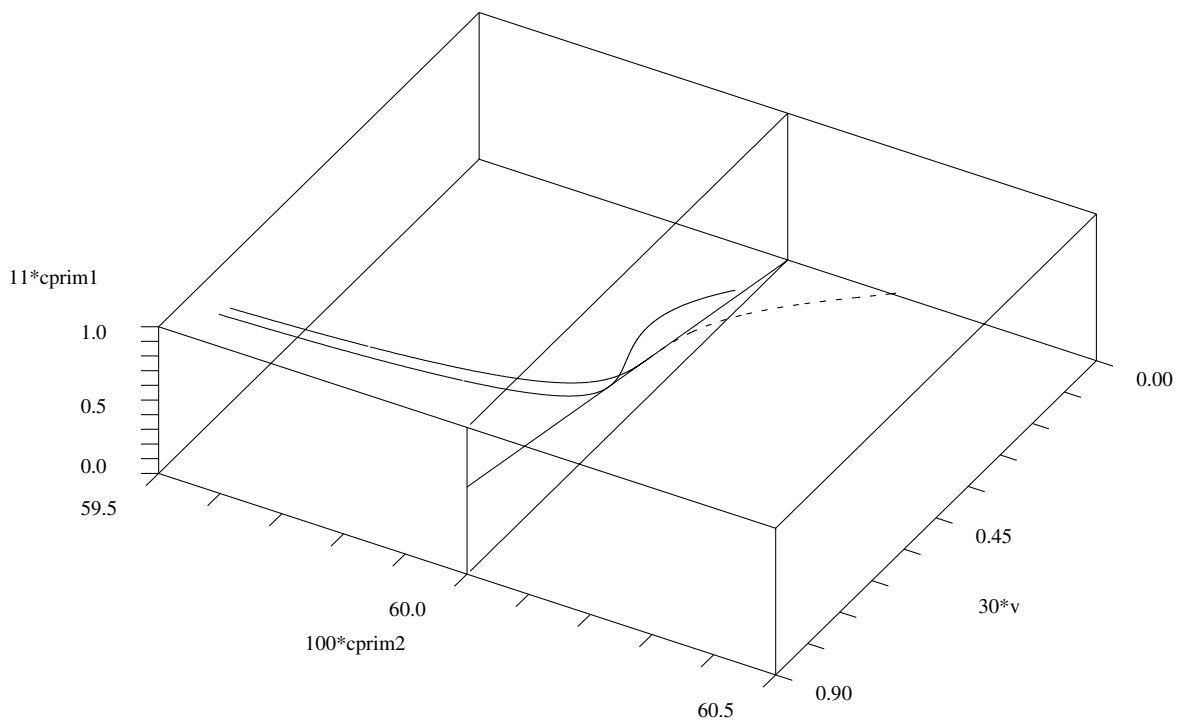

Figure 22 : exceptional trajectories

\section{REFERENCES}

[1] A. Agrachev: Symplectic methods in optimization and control. Geometry of feedback and optimal control, Marcel Dekker, New York, 1997. 


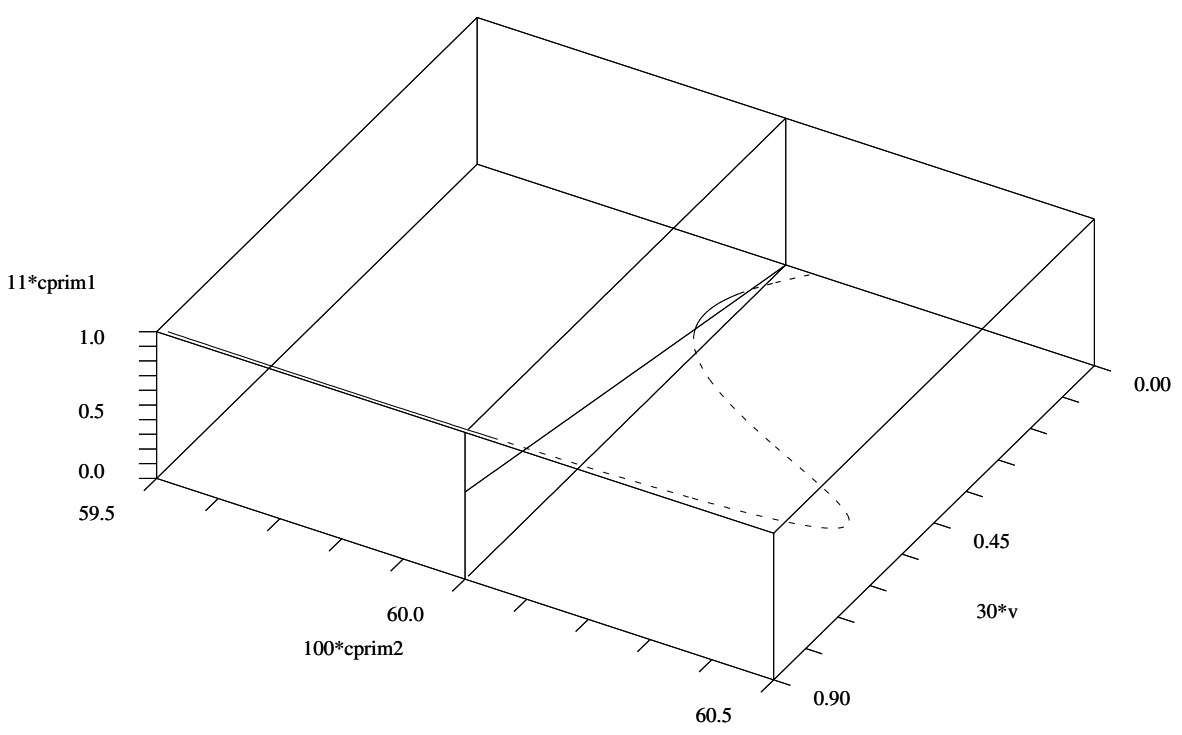

Figure $23: \gamma_{-}$trajectory near $\mathcal{E}_{-}$

[2] B. Bonnard: Rapport préliminaire sur la conduite optimale des réacteurs chimiques de type batch, Prépublication de l'Université de Bourgogne, Laboratoire de Topologie, $\mathrm{n}^{\circ} 58,1995$.

[3] B. Bonnard, J. de Morant: Towards a geometric theory in the time minimal control of chemical batch reactors, SIAM J. Contr. Opt., 33, 1995, 1279-1311.

[4] B. Bonnard, I. Kupka: Théorie des singularités de l'application entrée/sortie et optimalité des trajectoires singulières dans le problème du temps minimal, Forum mathematicum, 5, 1993, 111-159.

[5] B. Bonnard, G. Launay, M. Pelletier: Classification générique de synthèses temps minimales avec cible de codimension un et applications, Ann. IHP (an), 14, 1997, $55-102$.

[6] I. Ekeland: Discontinuité des champs hamiltoniens et existence de solutions optimales en calcul des variations, Pub. IHES, 47, 1977, 1-32.

[7] M. Feinberg: Chemical reaction network structure and stability of complex isothermal reactions, Chemical Engineering Sciences, 42, 1987, 2229-2268.

[8] H. Grauert, K. Fritzche: Several complex variables, Springer Verlag, New York, 1976.

[9] W. Harmon Ray: Advanced Process Control, Butterworths Reprint Series in chemical engineering, 1989.

[10] H. Hermes: Lie algebras of vector fields and local approximation of attainable sets, SIA M J. Control Opt., 16, 1978, 715-727.

[11] B. Jakubczyk: Critical Hamiltonians and feedback invariants. Geometry of feedback and optimal control, Marcel Dekker, New York, 1997.

[12] I. Kupka: Geometric theory of extremals in optimal control problems, I. The fold and Maxwell cases, Trans. Amer. Math. Soc., 299, 1987, 225-243.

[13] I. Kupka: Optimalité des extrémales ordinaires, Communication personnelle.

[14] G. Launay, M. Pelletier: The generic local structure of time-optimal synthesis with a target of codimension one in dimension greater than two, J. Dyn. Contr. Syst., 3, 1997, 165-204.

[15] L. Pontriaguine et al.: Théorie mathématique des processus optimaux, Mir, Moscou, 1974. 
[16] H.J. Sussmann: The structure of time-optimal trajectories for single-input systems in the plane: the $C^{\infty}$ non singular case, SIAM J. Control Opt, 25, 1987, 433-465.

[17] H.J. Sussmann: Regular synthesis for time-optimal control for single-input real analytic systems in the plane, SIAM J. Control Opt., 25, 1987, 1145-1162. 